\title{
Analysis of the Degradation in the Creep Strength of High-Cr Martensitic Steels
}

\author{
Manabu TAMURA ${ }^{1} \&$ Fujio $\mathrm{ABE}^{2}$ \\ ${ }^{1}$ 6-45-2 Hinominami, Konan-ku, Yokohama, 2340055, Japan \\ ${ }^{2}$ National Institute for Materials Science, 1-2-1 Sengen, Tsukuba 305-0047, Japan \\ Correspondence: Manabu TAMURA, 6-45-2 Hinominami, Konan-ku, Yokohama, 2340055, Japan. E-mail: \\ mtamura.1943@pep.ne.jp
}

Received: March 14, 2021

Accepted: July 30, 2021

Online Published: October 20, 2021

doi:10.5539/jmsr.v10n2p1

URL: https://doi.org/10.5539/jmsr.v10n2p1

\begin{abstract}
To investigate the formation process of the Z-phase, which lowers the long-term rupture strength of high-Cr martensitic steel, the creep curves of Grades T91, T92, and P92 were analyzed along with the experimental steels of $9 \mathrm{Cr}-1 \mathrm{~W}$ and $9 \mathrm{Cr}-4 \mathrm{~W}$ by applying an exponential law to the temperature, stress, and time parameters. The activation energy $(Q)$, activation volume $(V)$, and Larson-Miller constant $(C)$ were obtained as functions of creep strain. At the beginning of creep, sub-grain boundary strengthening occurs due to dislocations that are swept out of the sub-grains, which is followed by strengthening due to the rearrangement of $\mathrm{M}_{23} \mathrm{C}_{6}$ and the precipitation of the Laves phase. After $Q$ reaches a peak, heterogeneous recovery and subsequent heterogeneous deformation begin at an early stage of transient creep in the vicinity of several of the weakest boundaries due to coarsening of the precipitates. This activity triggers an unexpected degradation in strength due to the accelerated formation of the Z-phase. Stabilization of $\mathrm{M}_{23} \mathrm{C}_{6}$ and the Laves phase is important for mitigating the degradation of the long-term rupture strength of high-strength martensitic steel. The stabilization of the Laves phase is especially important for the Cr-Mo systems because $\mathrm{Fe}_{2} \mathrm{Mo}$ is easily coarsened at $\sim 600{ }^{\circ} \mathrm{C}$ as compared to $\mathrm{Fe}_{2} \mathrm{~W}$. Lowering the hardness and $\mathrm{Si}$ content also prevents excess hardening due to the Laves phase, which also mitigates the degradation. The online monitoring of creep curves and the $Q V C$ analysis render it possible to detect signs of long-term degradation under targeted conditions within a relatively short period.
\end{abstract}

Keywords: creep, exponential law, Grade 91, Grade 92, degradation mechanism, laves phase, Z-Phase

\section{Introduction}

Grades 91 and 92 steels, which were originally developed by Oak Ridge National Laboratory and Combustion Engineering Corporation (Sikka, Cowgill, \& Roberts, 1983) and Nippon Steel Corporation (Hasegawa, 2014), respectively, exhibit greatly improved efficiencies of power generation, thus reducing the contribution to global warming (Masuyama, 2001; Kimura, Sato, Bergins, Imano, \& Saito, 2011; Muroki, 2017). Over the years, since the development of these steels, several critical issues have surfaced. The most significant issues regarding these steels are their low rupture strength of the welded joints (Abson \& Rothwell, 2013) and the unexpected degradation of the base metal in long term rupture strength after creep test longer than several tens of thousands of hours (Kushima, Kimura, \& Abe, 1999; Sawada, Kushima, Kimura, \& Tabuchi, 2007). Regarding this unexpected reduction in strength of base metal, numerous studies have been undertaken with the aim of developing new alloys (Abe, 2006a; 2008; 2011; Hashizume, et al., 2009; Dudova, Plotnikova, Molodov, Belyyakov, \& Kaibyshev, 2012), estimating methods for long-term rupture strength (Tamura, 2015a; Maruyama, 2019), and investigating metallurgically the unexpected decline in strength which is overviewed by Abe (2006b). The formation of coarse Z-phase particles at the expense of the finely dispersed MX particles and recovery zones in the vicinity of the primary austenite grain boundaries (PAGBs) are considered as the dominant sources of the unexpected decline in strength (Kushima et al., 1999; Suzuki, Kumai, Kushima, Kimura, \& Abe, 2003; Sawada, Kushima, \& Kimura, 2006; Danielsen, 2007; Hald, 2008; Kimura, Sawada, Kushima, \& Toda, 2013). MX denotes the carbonitride with cubic structure, where $\mathrm{M}$ represents metallic elements such as $\mathrm{Nb}, \mathrm{V}$, and $\mathrm{Cr}$ and $\mathrm{X}$ represents $\mathrm{C}$ or $\mathrm{N}$, or both. The negative effect of $\mathrm{Ni}$ contained in the specification range of Grade 91 ( $\mathrm{Ni} \leq 0.40$ mass $\%$, hereinafter $\%$ ) on the long-term rupture strength during the formation of the Z-phase particles was reported by Kimura et al. (2013) and Sawada, Kushima, Hara, Tabuchi, and Kimura, (2014a). Sawada et al. (2019a) also recently demonstrated the 
harmful effect of the presence of micro-segregation prior to creep tests on the long-term rupture strength of ASME Grade T91. However, the following issues have not been properly elucidated: The number densities of the Z-phase particles in the Grade 91 ruptured specimens tested at $600{ }^{\circ} \mathrm{C}$ are larger than those of Grade 92 (Sawada et al., 2006), the strat time of the Z-phase formation reported by Sawada et al. (2007) does not coincide with the inflection point on the stress-rupture curves in high strength martensitic steels like Grade 91, which was highlighted by Yan, Wang, Shan, and Yang (2013). A proposal by Hu et al. (2009) and Di-Gianfrancesco, Vipraio, and Venditti (2013) suggests that the direct cause of the unexpected decline in rupture strength may be dependent on the stability of the Laves phase; however, this proposal is not generally accepted.

In this study, in order to resolve these issues, the creep behavior of high-Cr and high-strength martensitic steel was investigated from another perspective. Abe (2005) indicated from both the creep curves and the observation of the microstructures of $9 \mathrm{Cr}-\mathrm{W}$ steels ( $\mathrm{W}$ is $0,1,2$, or $4 \%$ ) that when the creep rate in a transient range of $9 \mathrm{Cr}-4 \mathrm{~W}$ steel decreases sharply at low stresses due to finely precipitated Laves phases on the boundaries, the coarsening of the Laves phase occurred rapidly. This rapid coarsening of the Laves phase resulted in the reduction of the rupture lives compared to the expected rupture lives based on the minimum creep rates $(M C R)$ at higher stresses. The rupture lives are strongly dependent on the creep behaviors, themselves. However, the creep curves of heat-resistant steels have not been extensively studied with respect to the rupture strength. The main reason for this is that systematic digital data of the creep curves of heat-resistant steels for practical uses have not been readily available compared to the time to rupture data. Recently, the National Institute for Materials Science (NIMS, Tsukuba, Japan) revised the Data Sheet (NIMS, No. 48B, 2018) of Grades T92 and P92. The data reported in Data Sheet No. 48B, the creep data of $\mathrm{P} 92$ reference code MJP, ranges from 550 to $750^{\circ} \mathrm{C}$ and the maximum rupture life reaches $106809 \mathrm{~h}$.

The aim of this study is to extend the knowledge about the unexpected decline in the rupture strength of high-strength martensitic steel by mainly analyzing creep curves of Grade P92. Similar alloy systems of Grades 91 and 92 are also investigated as references. Moreover, since the microstructural changes in these high-strength steels during long-term creep are very complex, the creep behavior of simple alloy systems, namely $9 \mathrm{Cr}-1 \mathrm{~W}$ and 9Cr-4W steels, is investigated (Tamura \& Abe; 2021). Three important parameters, the activation energy $(Q)$, activation volume $(V)$, and the Larson and Miller (L-M) constant $(C)$, (Larson \& Miller, 1952) for the long-term rupture data of typical heat-resistant steels for practical uses can be deduced by analyzing the rupture data using an exponential law (Tamura, Esaka, \& Shinozuka, 1999). The metallurgical meanings of these parameters, $Q, V$, and $C$, obtained using an exponential law are easily explained compared to the typical parameter of the stress exponent $(n)$ obtained using the commonly used power law. Some fundamental research findings have been obtained by analyzing creep data using an exponential law, such as microstructural changes during the long-term creep of typical heat-resistant steels (Tamura, Esaka, \& Shinozuka, 2000), the applicability of an exponential law in the creep of metals including nickel-based alloys and solders (Tamura, Esaka, \& Shinozuka, 2003), the analysis precipitates during creep of Grade 91 steel (Kabadwal, Tamura, Shinozuka, \& Esaka, 2010), the Larson-Miller constant of heat-resistant steel and its physical interpretation (Tamura, Abe, Shiba, Sakasegawa, \& Tanigawa, 2013), and changes in dislocation density during the creep of 9Cr-1W steel (Tamura \& Abe, 2015b). Moreover, the physical meaning of dislocation density in steady-state creep were investigated by analyzing the creep data of 21 types of ferritic/martensitic and austenitic steels including pure metals using an exponential law (Tamura, 2017). Sherby, Orr, and Dorn (1953) empirically demonstrated the well-known relationship that the creep rates of pure metals are controlled by the self-diffusion of each metal, which is also believed to be a major principle for interpreting the creep phenomena of complex alloy systems. Tamura (2017) used the steady-state creep rate using an exponential law to theoretically prove that Sherby's principle holds, even for complex alloy systems. As a result, it is evident that an exponential law is useful for understanding the creep phenomena of metals. Therefore, an exponential law is used for analyzing the creep data of the high-Cr martensitic steels in this study.

\section{Method of Analysis and Meanings of the Parameters $Q, V$, and $C$}

\subsection{Calculation of Time to Rupture}

Time to rupture of heat-resistant steel $\left(t_{r}\right)$ can be expressed as:

$$
t_{r}=t_{r 0} \exp \{(Q-\sigma V) / R T\}
$$

where, $R, T$, and $\sigma$ are the gas constant, absolute temperature $(\mathrm{K})$, and the applied normal tensile stress, respectively (Tamura et al., 1999). When the pre-exponential factor $\left(t_{r 0}\right)$ of Equation 1 is related to the well-known L-M constant $(C)$, the equation is given by:

$$
t_{r 0}=10^{-C}
$$


From Equations 1 and 2, we obtain

$$
\log t_{r}=Q / 2.3 R T-V / 2.3 R \cdot \sigma / T-C=[Q]-[V]-C
$$

Regression analysis of $\log t_{r}$ made as a function of $1 / T$ and $\sigma / T$ according to Equation 3, gives three parameters $Q, V$, and $C$ (hereinafter, $Q V C$ for all parameters). In Equation 3, $[Q]=Q / 2.3 R T$ and $[V]=$ $\sigma V / 2.3 R T$ are absolute numbers, which are used in subsequent sections. $Q$ and $V$ in Equation 1 are the apparent activation energy and the apparent activation volume for rupture, respectively. For simplicity, we refer to them as the activation energy and the activation volume, respectively. Usually, the stress vs. time to rupture data are plotted in a double log figure according to the Norton law (Norton, 1929). However, we assume Equation 1 and so, a linear relationship for the stress-time to rupture is obtained in a semi-logarithmic diagram (the y-axis is linear stress and the $\mathrm{x}$-axis is logarithm of the time to rupture, which is the same in the later sections). Tamura et al. (1999) confirmed that, for several heat-resistant steels, when long-term rupture data are classified into two or three groups based on test conditions, the rupture data for each group satisfies Equation 1. One of the technical merits of applying an exponential law to the rupture data is that it is easy to extrapolate the linear relationship obtained from a specific data group using Equation 1 in a semi-logarithmic diagram to a given test condition beyond the test conditions of the data group.

In Equation 1, we assume the average velocity of dislocations that glide through a crystal in a thermal activated process (Scheck, 1980; Tamura et al., 1999):

$$
v=v_{0} \exp \{-(Q-\sigma V) / R T\}
$$

and use the Orowan equation (Orowan, 1940):

$$
\dot{\varepsilon}=M \rho b v
$$

with the Monkman-Grant relationship (Monkman \& Grant, 1956):

$$
M C R=C_{M G} / t_{r}
$$

Here, $\dot{\varepsilon}, v, v_{0}, M, \rho, b, M C R$, and $C_{M G}$ are the creep rate, the average dislocation velocity, the pre-exponential factor of Equation 4, the factor conversion from shear strain to tensile strain, the dislocation density, the length of the Burgers vector, and the Monkman-Grant constant, respectively. We obtain from Equations 5 and 6 assuming $\dot{\varepsilon}=M C R$

$$
t_{r}=C_{M G} / M \rho b v .
$$

Therefore, it is comprehensible from Equations 1, 2, and 7 that although the dislocation density is inversely proportional to the time to rupture, it is generally accepted that the dislocation density together with the velocity are not treated as independent variables, but they are encompassed in the Larson-Miller constant when considering the time to rupture of heat-resistant steel. Here, we must also remind that the dislocation density for glide motions of dislocations corresponding to an observable creep strain, i.e., plastically deformed strain, is defined as the number of dislocations that are swept out of a crystal divided by the cross section of the crystal (Cottrell, 1964), and that the dislocation density is not equal to the dislocation density in the crystal, even though the dislocation density in the Orowan equation applies to all dislocations glided in a crystal, including dislocations in the crystal, because the Orowan equation is applicable to the general work-hardening theory.

\subsection{Broad Interpretation of Equation 1 to Time to a Specific Strain}

When we apply Equation 1 to time to a specific strain, the linear relationship between the $\sigma$ and logarithm of time to a specific strain for a given data group should be confirmed for a wide range of test conditions. Also, the linear relationship between $M C R$ and time to a specific strain in a double logarithmic diagram should be confirmed according to the Monkman-Grant relationship. We checked for all data studied, and among these typical examples will be shown in Appendix A, for simplicity.

In the Data Sheet of Grade 92, No. 48B (NIMS, 2018), an instantaneous strain, $\varepsilon_{0}$, time to $0.5,1,2,5 \%$ strain, and $t_{r}$ together with $M C R$ are listed and so, strain rate, $\dot{\varepsilon}\left(\varepsilon_{i}\right)$ at a strain of $\varepsilon_{i}$, where $i$ is the order number from the minimum strain reported, is calculated as

$$
\dot{\varepsilon}\left(\varepsilon_{i}\right)=\left(\varepsilon_{i}-\varepsilon_{i-1}\right) /\left(t_{i}-t_{i-1}\right)
$$

where $t_{i}$ is time to the $i^{\text {th }}$ strain. The accuracy of the strain rate calculated using Equation 8 is not so high, because strain intervals are rather large. Special attentions should be paid for creep rates near the $M C R$ because the strain rate near $M C R$ is rather small and the sign of an increase rate of strain rate changes from minus to plus with 
increasing creep time. Moreover, the creep rates just before rupture are also calculated in order to utilize the data of $t_{r}$ and rupture elongation (EL), even though the strain rates are largely affected by necking for $t_{i} / t_{r}>0.9$ (Lim et al., 2011). Concerning some of other steels studied, more data with small strain intervals are prepared, but only discrete data are adopted following the data structure of Grade 92 for comparison.

\subsection{Metallurgical meanings of $Q, V$, and $C$}

\subsubsection{Activation Energy}

The activation energy is the magnitude of the energy barrier that must be overcome for a dislocation to occur in an activation process, when we consider the model based on crystallography. Conversely, the activation energy calculated from Equation 3 is the apparent activation energy calculated from the measurable and macroscopic variables $T, \sigma$, and $t_{r}$ or $t_{i}$ and so, the value of $Q$ is obtained assuming that $C$ does not depend on $T$. Therefore, $Q$ contains not only the magnitude of the average creep resistance to mobile dislocations but also the effect of the temperature dependence of $C$ (Schoeck, 1980; Tamura et al., 2000). Moreover, $Q$ contains the effect of the back stress against a moving dislocation caused by grain boundaries, sub-boundaries, the stress field arising from nearby dislocations, and precipitates (Tamura, 2000). Therefore, when the sub-grains grow, the dislocation density decreases, and the precipitates are coarsened during long-term creep at high temperatures, the $Q$ decreases due to the reduction of the back stress. However, the value of $Q$ does not decrease without limits. It is established that the $Q$ s in high temperature creep of materials are almost equivalent to the activation energies for self-diffusion (Sherby et al., 1953; Tamura, 2017). Therefore, the lower limit of $Q$ approximately approaches the activation energy for self-diffusion of alpha-iron, $267 \mathrm{kJmol}^{-1}$ (Oikawa, 1982). Magnitude of the back stress can be roughly estimated using an exponential law and the details are explained in Appendix B.

\subsubsection{Larson-Miller Constant}

The $C$ can be calculated as a third term in Equation 3, and it is well-known that the value of $C$ is $\sim 20$ for many heat-resistant steels when the units for the test temperature and time to rupture are Kelvin and hours, respectively. In this study the value of $C$ is treated as an absolute value, and hereafter the explanation for the units of $C$ is omitted. The L-M constant is formulated as

$$
C=\log \left(\rho \lambda v_{e f f} b / 2 C_{M G}\right)+\Delta S / 2.3 R
$$

where $\lambda$ is the maximum distance that a dislocation can move from a starting point to the next stable position through the activation process, $v_{\text {eff }}$ is the effective attempt frequency per unit time to overcome the obstacles, and $\Delta S$ is the entropy change involved in the activation process (Tamura et al., 2013). The $\rho$ in Equation 9 is not the observable dislocation density in the sub-grains, $\rho_{o b}$, but refers to the dislocation density that contributes to the detectable creep strain as already explained with respect to Equation 5. However, we can assume that $\rho \sim \rho_{o b}$ at sufficiently high temperatures (Tamura et al., 2013; Tamura, 2017; Tamura, 2018). In many instances, the assumption of $C \sim 20$ is common in arranging data when sufficient data for optimizing the value of $C$ is not obtained. However, the value of $C$ largely affects the time to rupture according to Equations 1 and 2, because the time to rupture decreases by one order of magnitude when $C$ increases by 1 . Substituting $\rho=1 \mathrm{E} 12 \mathrm{~m}^{-2}$, $\lambda=8 \mathrm{E}-6 \mathrm{~m}, v_{e f f}=3600 \mathrm{E} 13 \mathrm{~h}^{-1}, C_{M G}=10(\%)$, and $b=2.5 \mathrm{E}-10 \mathrm{~m}$ into the first term in the right side of Equation 9, we obtain $\log \left(\rho \lambda v_{e f f} b / 2 C_{M G}\right)=12.7(\mathrm{~h}, \%)$. In this calculation, the logarithmic term only increases by 1 for an increase in $\rho$ by one order of magnitude. This change is not as large as the commonly accepted value, $C \sim 20$. In other words, the entropy term, $\Delta S / 2.3 R=20-12.7=7.3$, the second term of Equation 9 is quite large. Therefore, the main component of $C>20$ is considered to be contributed by the entropy change not only for the formation and migration of vacancies during creep controlled by self-diffusion, but also for the movement of dislocations.

Moving dislocations receive not only the applied stress but also the back stress arising from the surroundings. At the same time, the surroundings received the effect of the stress and strain field of the moving dislocations. However, these influences and the kinetic energy of the moving dislocations are irrelevant to the observed creep strain. Therefore, these energies are treated as heat loss in the system at a constant temperature when we analyze creep rate and time to rupture assuming a thermally activated process. This heat loss is accounted for in the formulation of the Gibbs free energy as the entropy change, i.e. as a part of $C$. The influence of the back stress is also accounted for in $Q$ as the barrier to be overcome. Therefore, both $Q$ and $C$ increase with increases in dislocation density and the formation of finer sub-structures. Consequently, the values of $Q$ and $C$ for martensitic steels are generally large, typically $Q=800 \mathrm{kJmol}^{-1}$ and $C=35$. Similarly, both $Q$ and $C$ are small when the dislocation density is low and the sub-grain size increases after long-term creep. In extreme cases, the value of $Q$ and $C$ for creep of a single crystal of pure iron are calculated as $294.0 \mathrm{kJmol}^{-1}$ and 12.06 , respectively from the 
analysis of creep data reported by Karashima, Iikubo, Watanabe, and Oikawa, (1971). The value of $Q$ and $C$ for creep of polycrystals of pure iron are calculated as $259.2 \mathrm{kJmol}^{-1}$ and 10.94 , respectively from creep data reported by Karashima, Iikubo, and Oikawa, (1972). Moreover, the values of $Q=259.2 \mathrm{kJmol}^{-1}$ and $C=18.56$ for $0.2 \%$ carbon steel (Tamura et al., 2013) and $Q=418 \mathrm{kJmol}^{-1}$ and $C=20.99$ as the average for 3 heats of $0.5 \mathrm{Cr}-0.5 \mathrm{Mo}$ steel (Tamura et al., 1999) are obtained. For practical purposes, $C \sim 20$ is confirmed for many heat resistant steels. On the contrary, the values of $C$ for creep of the martensitic heat-resistant steels studied are considerably larger, $C>30$. This is because martensitic steels contain many dislocations and possess very fine sub-structures. Therefore, energy loss, i.e., an increase in entropy, becomes very large for the movement of dislocations. Generally, the changes in the L-M constant $C$, can largely be regarded as the changes in the entropy term of $\Delta S / 2.3 R$ in Equation 9, when martensitic sub-structures are maintained, even after the long-term creep of high $\mathrm{Cr}$ martensitic steel with high strength.

\subsubsection{Activation Volume}

From Equation 3, $V$ is formulated as

$$
V=-2.3 R T\left(\partial \log t_{r} / \partial \sigma\right)_{T} .
$$

This indicates that the magnitude of $V$ is visually determined in a semi-logarithmic diagram of the $\sigma-\log t_{r}$ relationship, because $V$ is inversely proportional to the magnitude of the slope of the $\sigma-\log t_{r}$ relationship at a constant temperature. The term, $\sigma V$, in Equation 1 is equal to the work done by a specimen on a loading system in an activated state or the potential drop of the loading system (Esherby, 1956; Mura \& Mori, 1976). Also, the potential drop is equal to the force on a dislocation, $\tau b$, times the area swept out of the dislocation in an activated state, where $\tau$ is a shear stress on a slip plane and is proportional to $\sigma$. The $V$ generally increases after long-term creep, because the swept out area by a dislocation motion increases in the sub-grain when the size of the sub-grain increases or the densities of obstacles for a dislocation decrease due to recovery (Tamura et al., 2000).

\subsubsection{Inter-relationships among $Q, V$, and $C$}

Even though $Q$ is an important parameter for creep strength, it is not always true that the creep strength is high when $Q$ is large. This is because it is found from Equations 1 and 2 that the $t_{r}$ is the product of a very large value, i.e., $\exp \{(Q-\sigma V) / R T\}$ and a very small value of $10^{-C}$. Since $Q$ is large compared to $\sigma V$, then $Q$ and $C$ should have a strong positive correlation. Figure A1 in Appendix A shows a typical relationship between $Q$ and $C$ for Grade P92. As seen in Figure A1, the two parameters have a very strong positive correlation. However, it must be remembered that $Q$ and $C$ have the different meanings from each other. $Q$ denotes the magnitude of the barrier that must be overcome for a moving dislocation, that is caused by nearby precipitates, dislocations, boundaries, and the entire specimen. On the other hand, $V$ is also an important parameter in creep, but $V$ is a factor reflecting the movable area for a dislocation. Namely, $V$ is a parameter that only relates to the microstructures inside the sub-grains which is essentially different from $Q$.

\subsection{Heterogeneous Deformation}

In the previous sections we assumed uniform deformation, but Kushima et al. (1999) reported that the extremely recovered zone in the vicinity of the PAGBs with a width of approximately $1 \mu \mathrm{m}$ was formed in a ruptured specimen of Grade T91 tested at $600{ }^{\circ} \mathrm{C}$ under $100 \mathrm{MPa}\left(t_{r}=34141 \mathrm{~h}\right)$, where the unexpected drop in rupture strength was clearly confirmed. Strain concentration should occur in these heterogeneously recovered zones (HRZ). The strain rate in the HRZ is expected to become higher than the observed creep rate, and consequently, the stress concentration should occur around or within the HRZ. The "time to rupture" corresponding to the higher strain rate in the HRZ decreases according to the Monkman-Grant relationship (Monkman \& Grant, 1956). In this case, if we assume that $Q$ and $V$ do not change, the value of $C$ increases according to Equations 1 and 2, and it is clear that $V$ decreases with stress concentration in the HRZ from Equation 1 assuming that the other variables are constant. These changes are more easily understood in cases using Equation 1. Table 1 shows the calculated results for six cases of heterogeneous deformation using a data set of rupture lives: Case A; standard conditions with temperature interval $\Delta T=50 \mathrm{~K}$, Case B; strain concentration $\left(t_{r}=0.1 \mathrm{~A}\right)$, Case $\mathrm{C}$; stress concentration $(\sigma=$ $1.5 \mathrm{~A})$, case $\mathrm{D}$; stress and strain concentrations $\left(t_{r}=0.1 \mathrm{~A}, \sigma=1.5 \mathrm{~A}\right)$, Case $\mathrm{E}$; the occurrence of remarkable and heterogeneous recovery simulated by increasing the temperature interval $(\Delta T=\mathrm{A}+10 \mathrm{~K})$, which decreases $Q$, and Case $\mathrm{F}$; stress and strain concentrations with remarkable and heterogeneous recovery $\left(t_{r}=0.1 \mathrm{~A}, \sigma=\right.$ $1.5 \mathrm{~A}, \Delta T=\mathrm{A}+10 \mathrm{~K}$ ), where A means the corresponding test conditions of the standard Case A, compared to each studied case. It is found from Table 1 , that $V$ decreases in Cases $\mathrm{C}$ and $\mathrm{D}$, whereas $C$ increases in Cases B and $\mathrm{D}$ compared to Case A and when the temperature interval increases in Case $\mathrm{E}$, both $Q$ and $C$ decrease. Finally when we assume the occurrence of the stress and strain concentration in the HRZ, i.e., Case F, all of QVC 
clearly decrease compared to the uniform deformation seen in Case A. Case F is set up assuming the formation of the extremely recovered zone along the PAGBs observed by Kushima et al. (1999) in a ruptured specimen, the time to rupture of which becomes unexpectedly short. Consequently, time to rupture for Case $\mathrm{F}$ is shorter than that of Case A.

\subsection{Rupture Strength of Welded Joints}

Typical creep rupture behavior of a heterogeneously recovered and deformed zone can be observed in the creep tests of the welded joints. Figure 1 shows the results of rupture tests of the welded joints made of Grade 91 plate ( $25 \mathrm{~mm}$ thickness) fabricated by tungsten inert gas (TIG) welding and post weld heat treatment (PWHT) at $745{ }^{\circ} \mathrm{C}$ for $60 \mathrm{~min}$ (Hongo, Tabuchi, Li, \& Takahashi, 2009). In the figure, the results of the base metal and the simulated heat affected zone (HAZ) with the same PWHT as the TIG welded joint are also shown. The TIG welded joint specimens are ruptured at the HAZ except for two specimens tested at $550{ }^{\circ} \mathrm{C}$ under two higher stresses. The simulated $\mathrm{HAZ}$ specimens are rapidly heated to the approximate temperature of the $\mathrm{A}_{\mathrm{C} 3}$ transformation $\left(\sim 900^{\circ} \mathrm{C}\right)$ followed by rapid cooling, where the whole specimen is heat-treated to simulate the microstructure of the HAZ of TIG welding, which minimizes the rupture lives of the simulated HAZ. Therefore, it is generally accepted that the rupture lives of the welded joint lies between those of the base metal and that of the simulated HAZ. Moreover, the reliability of the tests is also confirmed from those of the rupture lives of the welded joint which ruptured at the base metal under the two higher stresses which are similar to those of the base metal. The $Q V C$ s of the welded joint ruptured at the HAZ, the base metal, and the simulated HAZ are calculated according to Equation 3, and the results are listed in Table 2. It is seen in Table 2 that the value of $Q$ for the simulated HAZ is higher than that of the base metal, but at the same time $C$ of the simulated HAZ increases much more than that of the base metal. As a result, the rupture lives of the simulated HAZ become shorter than those of the base metals. Most importantly, it is seen in Table 2 that the $Q V C$ of the welded joints are smaller than those of the base metal and this is in agreement with the results for the Case $\mathrm{F}$ in Table 1, where the microstructure is assumed the formation of the extremely recovered zone along the PAGBs as reported by Kushima et al. (1999).

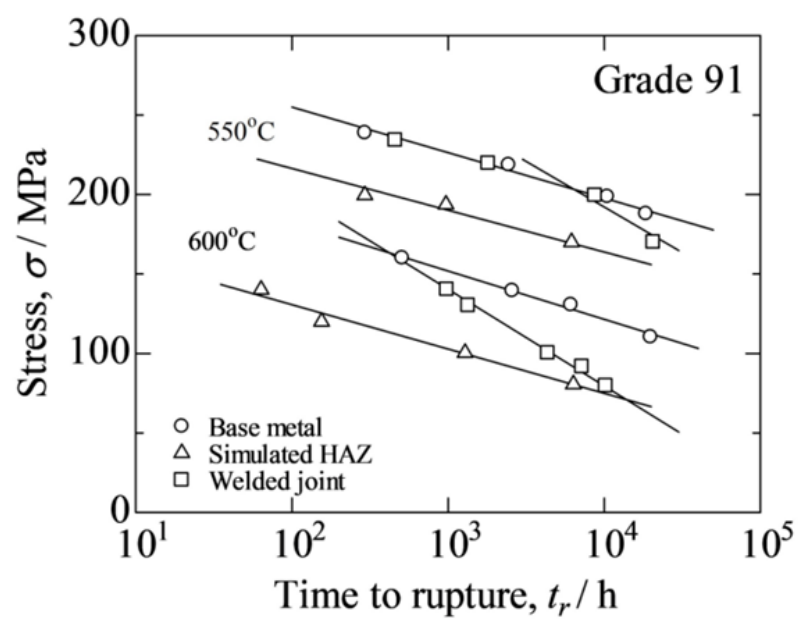

Figure 1. Stress-rupture life relationship for TIG welded joint, the simulated HAZ, and the base metal of Grade 91

Hongo et al. (2009) also performed the rupture tests of the TIG welded joint at $650{ }^{\circ} \mathrm{C}$, and a similar result is obtained using the data at 600 and $650^{\circ} \mathrm{C}$. It is confirmed by the data of Ogata, Sakaki, and Yaguchi (2009) that the QVC of the gas tungsten arc welded (GTAW) joint ruptured at the HAZ of Grade P91 are smaller than those of the simulated HAZ and the base metal, which is further confirmed by the data of Tabuchi et al. (2001) in which the $V$ of the GTAW joint and the electron beam welding (EBW) joint of Grade P122 at $650{ }^{\circ} \mathrm{C}$ are smaller than the base metal and the simulated HAZ, although the $Q$ and $C$ cannot be calculated. Morimoto, Ohkita, and Sakurai (1997) also reported the rupture lives of the welded joints of Grade 92 and the $Q V C$ calculated from the rupture data were smaller than the base metal. These rupture phenomena of the welded joints are the typical cases of heterogeneous deformation. Therefore, it is phenomenologically understood from the above that the all parameters of $Q V C$ for the heterogeneous deformation become smaller than those of the base metals which exhibit uniform deformation. 
Table 1. Results of $Q V C$ calculation for six test conditions (explained in the text). Bold-italicized numbers indicate the changes from the standard test condition, Case A

\begin{tabular}{ccccccc}
\hline \multirow{2}{*}{ Conditions } & $\mathrm{A}$ & $\mathrm{B}$ & $\mathrm{C}$ & $\mathrm{D}$ & $\mathrm{E}$ & $\mathrm{F}$ \\
& standard $(\Delta T=50 \mathrm{~K})$ & $t_{r}=0.1 \mathrm{~A}$ & $\Delta=1.5 \mathrm{~A}$ & \multicolumn{1}{c}{$\mathrm{B}^{*} \mathrm{C}$} & Increase in $\Delta T,(\Delta T=60 \mathrm{~K})$ & $\mathrm{E}^{*} \mathrm{~B}^{*} \mathrm{C}$ \\
\hline$Q / \mathrm{kJmol}^{-1}$ & 485.5 & 485.5 & 485.5 & 485.5 & $\mathbf{4 0 8 . 8}$ & $\mathbf{4 0 8 . 8}$ \\
$V / \mathrm{cm}^{3} \mathrm{~mol}^{-1}$ & 365.8 & 365.8 & $\mathbf{2 4 3 . 9}$ & $\mathbf{2 4 3 . 9}$ & 365.8 & $\mathbf{2 4 3 . 9}$ \\
$C$ & 22.48 & $\mathbf{2 3 . 4 8}$ & 22.48 & $\mathbf{2 3 . 4 8}$ & $\mathbf{1 8 . 1 3}$ & $\mathbf{1 9 . 1 3}$ \\
\hline
\end{tabular}

Table 2. The results of the QVC analyses on the rupture data shown in Figure 1 of the TIG welded joint, the simulated HAZ, and the base metal of Grade 91

\begin{tabular}{cccc}
\hline Grade 91 & Base metal & Simulated. HAZ & Welded joint ruptured at HAZ \\
\hline$Q / \mathrm{kJmol}^{-1}$ & 798.3 & 974.2 & 561.4 \\
$V / \mathrm{cm}^{3} \mathrm{~mol}^{-1}$ & 549.7 & 598.8 & 274.6 \\
$C$ & 39.82 & 51.67 & 28.32 \\
\hline
\end{tabular}

\section{Materials Analyzed and Their Microstructure}

\subsection{Materials Analyzed}

Five kinds of steels were analyzed: 9Cr-1W steel (Abe, Araki, \& Noda, 1991; Abe, 2003), 9Cr-4W steel (Abe et al., 1991; Abe, Nakazawa, Araki, \& Noda, 1992, Abe, 2005), Grade T91 (NIMS, 2007; 2014), and Grades T92 and P92 (NIMS, 2018). Table 3 shows the form of the product, heat treatment conditions, chemical composition, and hardness of the materials tested, and the precipitates before and during the creep tests are also listed in the table. The $9 \mathrm{Cr}-1 \mathrm{~W}$ and $9 \mathrm{Cr}-4 \mathrm{~W}$ steels are prepared in a laboratory scale. MGC, MJP, and MJT in Table 3 are the reference code numbers for a single heat used in NIMS. The names of $9 \mathrm{Cr}-1 \mathrm{~W}, 9 \mathrm{Cr}-4 \mathrm{~W}, \mathrm{~T} 91 / \mathrm{MGC}, \mathrm{T} 92 / \mathrm{MJT}$, and P92/MJP are used for the steels in this study, and moreover, the abbreviated names, $1 \mathrm{~W}, 4 \mathrm{~W}, \mathrm{MGC}$, MJP, and MJT, are also used in some cases. When we refer to previous work or generally describe the scientific facts, we use Grade T91 or P91 and Grade T92 or P92 according to the ASME standard. The major differences between P92/MJP and T92/MJT are the hardness and Si content; MJP is rather soft compared to MJT and the Laves phase is considered to be less formed in MJP, because the Si content of MJP is smaller than that of MJT (Hosoi, Wade, Kunimitsu, \& Urita, 1986).

\subsection{9 $r$-W Steel}

The microstructure of the quenched and tempered $9 \mathrm{Cr}-1 \mathrm{~W}$ is tempered martensite, and the precipitates before the rupture test and during the creep test are only $\mathrm{M}_{23} \mathrm{C}_{6}$ and the Laves phase is not confirmed (Abe et al., 1991; Abe, 2005), where $\mathrm{M}$ denotes the metallic element such as $\mathrm{Cr}, \mathrm{Fe}, \mathrm{Mo}$, and $\mathrm{W}$. The microstructure of quenched and tempered $9 \mathrm{Cr}-4 \mathrm{~W}$ is mainly tempered martensite but containing $10 \mathrm{vol} \%$ of delta ferrite (Abe et al., 1991). The $9 \mathrm{Cr}-4 \mathrm{~W}$ steel contains $\mathrm{M}_{23} \mathrm{C}_{6}$ and a small amount of $\mathrm{M}_{6} \mathrm{C}$ where $\mathrm{M}$ denotes $\mathrm{Fe}$ and $\mathrm{W}$ and the Laves phase was not confirmed before the creep tests. During transient creep at 600 and $650{ }^{\circ} \mathrm{C}$, fine Laves phase precipitates were observed on the lath martensite boundaries, which contribute to strengthen the grain- and sub-boundaries together with the $\mathrm{M}_{23} \mathrm{C}_{6}$ precipitates. However, the Laves phase precipitates are soon coarsened and consequently accelerate the creep rate (Abe, 2005). The nose point of a time-temperature-precipitation (TTP) curve for the Laves phase in the delta ferrite of aged specimens is $650^{\circ} \mathrm{C}$ and $15 \mathrm{~h}$ (Abe et al., 1991; Abe et al., 1992).

\subsection{Grade 91 Steel}

$\mathrm{M}_{23} \mathrm{C}_{6}$ particles are observed on the PAGB, packet, block, and lath boundaries, two kinds of fine $\mathrm{MX}$ with $\mathrm{Nb} \sim 90 \%$ and $\mathrm{V} \sim 70 \%$ for each are observed on the boundaries and inside the sub-grains in T91 steel before the test (Suzuki, Kumai, Kushima, Kimura, \& Abe, 2000; Sawada et al., 2006). $\mathrm{M}_{23} \mathrm{C}_{6}$ particles grow gradually during creep, the average sizes are 250 and $450 \mathrm{~nm}$ in the specimens ruptured at $600{ }^{\circ} \mathrm{C}$ for $34141 \mathrm{~h}$ and $650{ }^{\circ} \mathrm{C}$ for $41452 \mathrm{~h}$, respectively. The chemical compositions of $\mathrm{M}_{23} \mathrm{C}_{6}$ are enriched with $\mathrm{Cr}$ which increases slightly the creep time, and the amount of precipitates increases slightly during creep (Suzuki et al., 2000; Suzuki et al., 2003). Finely dispersed MX particles in T91 remain considerably stable for a long duration and the average sizes are approximately 40 and $60 \mathrm{~nm}$ in the ruptured specimens at $600{ }^{\circ} \mathrm{C}$ for $34141 \mathrm{~h}$ and $650{ }^{\circ} \mathrm{C}$ for $41452 \mathrm{~h}$, respectively (Suzuki et al., 2000), but the number density of $\mathrm{NbX}$ begins to diminish from about $1000 \mathrm{~h}$ and 
disappears after $41542 \mathrm{~h}$ at $650^{\circ} \mathrm{C}$ (Suzuki et al., 2003). Laves phase particles are observed in the aged specimens at a wide temperature range of $500-650^{\circ} \mathrm{C}$ and the nose point of the TTP curve for the Laves phase is $575{ }^{\circ} \mathrm{C}$ and $\sim 1700 \mathrm{~h}$ (Tamura, Hayakawa, Yoshitake, Hishinuma, \& Kondo, 1988). Suzuki et al. (2000) reported on the Laves phase in Grade T91 employing an X-ray diffractometer (XRD) and a transmission electron microscope (TEM): The Laves phase is formed even after a short time duration $(40 \mathrm{~h})$ at $600{ }^{\circ} \mathrm{C}$ and the amount of precipitates becomes approximately constant after $10000 \mathrm{~h}$. The growth rate of the Laves phase is fast, and the size reaches $\sim 1000 \mathrm{~nm}$ at $600{ }^{\circ} \mathrm{C}$ for $34141 \mathrm{~h}$, however, the Laves phase does not form at $650^{\circ} \mathrm{C}$. Although the precipitation cites of most of Laves phase in a long-term ruptured specimen of Grade P91 are PAGB and sub-boundaries (Panait, Bendick, Fuchsmann, Gourgues-Lorezon, \& Besson, 2010a), Sawada et al. (1999) highlighted that the fine Laves phase also precipitates on the lath boundaries. Yan et al. (2013) also reported similar results for $10 \mathrm{Cr}$ martensitic steel. However, systematic research work on the precipitation behavior of the Laves phase in an early creep stage of Grade 91 is lacking in the literature.

Table 3. Materials analyzed and their characteristics. The chemical composition is given in mass $\%, 9 \mathrm{Cr}-4 \mathrm{~W}$ contains $10 \%$ delta ferrite, and the hardnesses of T91, T92, and P92 are converted from HRC

\begin{tabular}{cccccc}
\hline Steel & $9 \mathrm{C}-1 \mathrm{~W}$ & $9 \mathrm{Cr}-4 \mathrm{~W}$ & T91/MGC & T91/MJT & P92/MJP \\
\hline Product form & Rod & Rod & Tube & Tube & Pipe \\
Austenitizing $/{ }^{\circ} \mathrm{C}$ & 950 & 1000 & 1050 & 1100 & 1070 \\
Tempering $/{ }^{\circ} \mathrm{C}$ & 750 & 800 & 765 & 780 & 780 \\
$\mathrm{Hv}$ & 203 & 211 & 230 & 222 & 213 \\
& & & & 0.098 & 0.11 \\
$\mathrm{C}$ & 0.10 & 0.10 & 0.09 & 0.29 & 0.10 \\
$\mathrm{Si}$ & 0.29 & 0.29 & 0.29 & 0.13 & 0.17 \\
$\mathrm{Ni}$ & - & - & 0.28 & 9.50 & 9.26 \\
$\mathrm{Cr}$ & 9.01 & 9.09 & 8.70 & 0.36 & 0.42 \\
$\mathrm{Mo}$ & - & - & 0.90 & 1.74 & 1.67 \\
$\mathrm{~W}$ & 0.99 & 3.93 & - & 0.19 & 0.16 \\
$\mathrm{~V}$ & - & - & 0.22 & 0.062 & 0.057 \\
$\mathrm{Nb}$ & - & - & 0.072 & 0.0462 & 0.0462 \\
$\mathrm{~N}$ & 0.002 & 0.002 & 0.044 & 0.002 & 0.002 \\
$\mathrm{~B}$ & - & - & - & \multicolumn{2}{c}{$\mathrm{M}_{23} \mathrm{C}_{6}, \mathrm{MX}, \mathrm{BN}$} \\
Before test & $\mathrm{M}_{23} \mathrm{C}_{6}$ & $\mathrm{M}_{23} \mathrm{C}_{6}, \mathrm{M}_{6} \mathrm{C}$ & $\mathrm{M}_{23} \mathrm{C}_{6}, \mathrm{MX}$ & $\mathrm{M}_{23} \mathrm{C}_{6}, \mathrm{MX}$, Laves phase, Z-phase, BN \\
During creep & $\mathrm{M}_{23} \mathrm{C}_{6}$ & $\mathrm{M}_{23} \mathrm{C}_{6}$, Laves phase & $\mathrm{M}_{23} \mathrm{C}_{6}, \mathrm{MX}$, Laves phase, Z-phase
\end{tabular}

Formation boundaries of the Z-phase larger than $20 \mathrm{~nm}$ determined using ruptured specimens of Grade T91 are approximately 40000,10000 , and $4000 \mathrm{~h}$ at 550,600 , and $650{ }^{\circ} \mathrm{C}$, respectively, and longer than $30000 \mathrm{~h}$ at $700{ }^{\circ} \mathrm{C}$ (Sawada et al., 2007). Z-phase precipitates appear in ruptured specimens at 600 and $650{ }^{\circ} \mathrm{C}$ of Grade T91 related to a decrease in the density of the finely dispersed MX particles (Suzuki et al., 2003). Sawada, Kushima, Tabuchi, and Kimura (2011) also clarified using creep interrupted specimens tested at $600{ }^{\circ} \mathrm{C}$ under $70 \mathrm{MPa}$ that the number density of MX particles decreases from $8 \mathrm{E} 12 \mathrm{~m}^{-2}$ before the creep test to $7.3 \mathrm{E} 11 \mathrm{~m}^{-2}$ at rupture $\left(t_{r}=80736.8 \mathrm{~h}\right)$ related to an increase in the number density of the Z-phase precipitates from $2.2 \mathrm{E} 10 \mathrm{~m}^{-2}$ for $1000 \mathrm{~h}$ creep exposure to the approximately the same level of $6.3 \mathrm{E} 11 \mathrm{~m}^{-2}$ at rupture. Formation of the Z-phase is accelerated by creep strain or stress, because the number density of the Z-phase is $3.3 \mathrm{E} 11 \mathrm{~m}^{-2}$ for the gauge portion of a ruptured specimen $\left(600{ }^{\circ} \mathrm{C}, 34141 \mathrm{~h}\right)$, and is larger than that of the grip portion of $7.8 \mathrm{E} 10 \mathrm{~m}^{-2}$ (Sawada et al., 2006). The Z-phase particles in Grade T91 precipitate mainly on the PAGB and packet boundaries (Suzuki et al., 2000; Sawada et al., 2006; Sawada et al., 2007) and the average sizes are $111 \mathrm{~nm}$ at $600{ }^{\circ} \mathrm{C}$ and $t_{r}=34141 \mathrm{~h}$ and $533 \mathrm{~nm}$ at $650{ }^{\circ} \mathrm{C}$ and $t_{r}=41452 \mathrm{~h}$ (Suzuki et al., 2003). The average chemical compositions of the Z-phase of Grade T91 lie within a narrow range of $40-44 \% \mathrm{Cr}, 19-27 \% \mathrm{Nb}$, and $32-35 \%$ V (Suzuki et al., 2003; Sawada et al., 2006; 2007). Kocer, Abe, and Soon (2009) reported that the 
driving force of the Z-phase formation is large in martensitic steel with high contents of $\mathrm{Cr}, \mathrm{Nb}$, and $\mathrm{N}$ and so, Grade 91 forms a lower quantity of the Z-phase compared to Gr. 122. Sawada et al. (2014a) reported that Grade T91 with a high Ni content of $0.28 \%$ easily forms the Z-phase compared with that containing $0.04 \% \mathrm{Ni}$.

It has been confirmed by analyzing the microstructures of the long-term ruptured specimens that the number density of MX particles in the vicinity of the PAGBs decreases, the Z-phase particles grow mainly near the PAGBs consuming nearby MX particles, the coarse precipitates of the Z-phase, $\mathrm{M}_{23} \mathrm{C}_{6}$, and the Laves phase are formed on and near the PAGBs, and as a result, extremely recovered zones along the PAGBs are formed. Moreover, these recovery zones with a reduction in the strengthening factor of the MX particles begin to form from time to the $M C R$, which results in the unexpected drop in the rupture strength (Kushima et al., 1999; Kimura, Suzuki, Toda, Kushima, \& Abe, 2002; Sawada et al., 2011).

\subsection{Grade 92 Steel}

The microstructure before the creep test is similar to that of Grade 91 except for BN (Hasegawa et al., 2004; Sawada et al., 2006). Ennis, Zielinska-Lipiec, Wachter, and Czyrska-Filemonowicz (1997) reported that the chemical composition of $\mathrm{M}_{23} \mathrm{C}_{6}$ in a ruptured specimen of $\mathrm{P} 92$ at $600{ }^{\circ} \mathrm{C}$ and $t_{r}=9755 \mathrm{~h}$ is $46 \% \mathrm{Cr}-24 \% \mathrm{Fe}-27 \% \mathrm{~W}-2.2 \% \mathrm{Mo}-0.9 \% \mathrm{~V}$. Conversely, Nie et al. (2014) report that the chemical composition of $\mathrm{M}_{23} \mathrm{C}_{6}$ for $\mathrm{T} 92$ at $600{ }^{\circ} \mathrm{C}$ and $t_{r}=8472 \mathrm{~h}$ is $60 \% \mathrm{Cr}-34 \% \mathrm{Fe}-4.3 \% \mathrm{~W}-2 \% \mathrm{Mo}$. The value of $27 \%$ of $\mathrm{W}$ reported by Ennis is not only much larger than that reported by Nie but is also still larger than the value of $15.6 \% \mathrm{~W}$ which is estimated from the chemical composition of the extracted residues of a quenched and tempered state of $10 \mathrm{Cr}-1.8 \mathrm{~W}-\mathrm{NbVNB}$ steel reported by Azuma, Miki, Tanaka, and Ishiguro (2002). It is surprising that even the chemical composition of the stable precipitates of $\mathrm{M}_{23} \mathrm{C}_{6}$ fluctuates depending on the state of the analyzed samples. The average size of $\mathrm{M}_{23} \mathrm{C}_{6}$ in P92 increases moderately from $140 \mathrm{~nm}$ before the test to $210 \mathrm{~nm}$ at $600{ }^{\circ} \mathrm{C}$ for $t_{r}=6718 \mathrm{~h}$ (Jiang, Zhu, \& Wang, 2013) and these values are similar to those for T91 (Suzuki et al., 2003). On the other hand Nie et al. (2014) reported that the average sizes of $\mathrm{M}_{23} \mathrm{C}_{6}$ in a gauge and grip portion creep-tested at $700{ }^{\circ} \mathrm{C}$ for $t_{r}=8232 \mathrm{~h}$ are approximately $230 \mathrm{~nm}$ in both cases and these values are equivalent to the value at $650{ }^{\circ} \mathrm{C}$ for the corresponding creep time of T91 (Suzuki et al., 2003). Haetterstrand and Andren (2001) also reported that the average sizes of $\mathrm{M}_{23} \mathrm{C}_{6}$ in the aged specimens of $\mathrm{P} 92$ for $26000 \mathrm{~h}$ at 600 and $650{ }^{\circ} \mathrm{C}$ are 110 and $150 \mathrm{~nm}$, respectively, which indicates that the size of $\mathrm{M}_{23} \mathrm{C}_{6}$ of Grade 92 is smaller than that of Grade 91 and the size in a gauge portion is larger than those of the specimens without creep deformation.

Precipitation of the Laves phase in $\mathrm{P} 92$ begins within a short time of aging at 600 and $650{ }^{\circ} \mathrm{C}$ and the amounts become approximately constant by $10000 \mathrm{~h}$ (Haetterstrand \& Andren, 2001; Zielinski, Golanski, \& Sroka, 2016). The growth rate of the Laves phase is high and the sizes become approximately 200 and $300 \mathrm{~nm}$ in the necked portions of the ruptured specimens tested at 600 and $649^{\circ} \mathrm{C}$ for about $5000 \mathrm{~h}$, respectively (Nie et al., 2014). Much larger sizes of approximately 300 and $700 \mathrm{~nm}$ are reported for the aged specimens of $\mathrm{P} 92$ at 600 and $650{ }^{\circ} \mathrm{C}$ for 5000 h, respectively (Haetterstrand \& Andren, 2001). The precipitation site of the Laves phase after long-term creep is mainly on the PAGBs (Yan et al., 2013). These massive Laves phase particles are frequently observed in $\mathrm{P} 92$ near the coarse $\mathrm{M}_{23} \mathrm{C}_{6}$ particles (Dimmler, Weinert, Kozeschnik, \& Cerjak, 2003; Dudko, Belyakov, Molodov, \& Kaibyshev, 2013; Nie et al., 2014; Xu et al., 2015; Maddi et al., 2016). However, Hasegawa, Ohgami, and Muraki (2003) and also Hasegawa et al. (2004) reported that the Laves phase together with $\mathrm{M}_{23} \mathrm{C}_{6}$ can strengthen the block boundaries during creep of Grade P92. Kipelova, Belyakov, and Kaibyshev (2012) confirmed that fine Laves phase particles are formed on the lath boundaries of the crept specimen by $1 \%$ strain at $650{ }^{\circ} \mathrm{C}\left(t_{1}=374 \mathrm{~h}\right)$ of P911(3Co) steel. Moreover, Zeng, Jia, Cai, Dong, and Wang (2018) recently reported that the Laves phase not only forms on the PAGB and lath boundaries but also finely precipitates inside the lath martensite at a scale of 100 $\mathrm{nm}$ order for a reheater tube of $\mathrm{P} 92$ which served for $9854 \mathrm{~h}$ in an actual power plant operated at $603{ }^{\circ} \mathrm{C}$ and under $5.87 \mathrm{MPa}$.

MX particles composed of fine NbX type and very fine VX type with a size below few 10s of nm are distributed uniformly in Grade P92 before the creep test (Hasegawa et al., 2004; Sawada et al., 2006; Danielsen et al., 2007; Zeng et al., 2018). However, MX particles are coarsened after creep deformation at $600{ }^{\circ} \mathrm{C}$ for $t_{r}=39534 \mathrm{~h}$ and especially, coarse MX particles with a size over $100 \mathrm{~nm}$ are observed in the vicinity of the PAGBs (Sawada et al., 2006). Yoshizawa et al. (2009) reported that NbX type particles disappear and only VX type particles are left after aging at $650{ }^{\circ} \mathrm{C}$ for $21000 \mathrm{~h}$ in an equivalent Grade 92 steel and for $10000 \mathrm{~h}$ in an equivalent Grade 122 steel and then the Z-phase is formed. This phenomenon of the disappearance of the NbX type particles is also reported in Grade 91 (Suzuki et al., 2003). As mentioned above, it is well accepted by many researchers (Sawada et al., 2006; Danielsen et al., 2007; Yan et al., 2013; Di-Gianfrancesco et al., 2013; Sawada \& Kimura, 2019b) with respect to the formation of the Z-phase particles that the coarsening of the Laves phase is relating to the decrease in the number density or disappearance of a potent strengthening factor, MX, and the promotion of the Z-phase 
formation consuming MX occurs sequentially, which results in the unexpected drop in creep strength. However, research on the systematic changes in chemical composition and size of these precipitates during creep of Grade 92 is lacking.

Haetterstrand and Andren (2001) reported a small number of Z-phase particles in P92 aged $26000 \mathrm{~h}$ at 600 and $650{ }^{\circ} \mathrm{C}$ employing TEM. This may be the first report on the Z-phase in P92, because these aging conditions correspond well to the aging conditions required for the appearance of the Z-phase and the Z-phase is confirmed using a selected area diffraction pattern technique (Yoshizawa et al., 2009). Sawada et al. (2006) reported that a large number of Z-phase particles with an average size of $155 \mathrm{~nm}$ and chemical composition of $44 \% \mathrm{Cr}-35 \% \mathrm{~V}-16 \% \mathrm{Nb}-5 \% \mathrm{Fe}$ are formed on the PAGBs and packet boundaries in a ruptured specimen at $600{ }^{\circ} \mathrm{C}$ $\left(t_{r}=39534 \mathrm{~h}\right)$ of T92 accompanying a decrease in the number density of MX, and that the Z-phase is found in a ruptured specimen longer than $\sim 8000 \mathrm{~h}$ at $600{ }^{\circ} \mathrm{C}$, but the number densities of the Z-phase in T92 are one order of magnitude lower than those of T91. Danielsen (2007) proposed a model for the formation of the Z-phase in which the nuclei of the Z-phase are formed by diffusion of $\mathrm{Cr}$ into $\mathrm{NbX}$ particles and grow consuming surrounding MX particles, which leads to the unexpected drop in the long-term rupture strength. It was also reported that there are two kinds of Z-phase, a tetragonal one with a high $\mathrm{Cr}$ content and cubic one with a lower $\mathrm{Cr}$ content and that the $\mathrm{Z}$-phase is even formed without $\mathrm{Nb}$. Formation boundaries of the Z-phase larger than the $20 \mathrm{~nm}$ evaluated using ruptured specimens of Grade T92 are $\sim 25000,8000,3500$, and $10000 \mathrm{~h}$ at $550,600,650$, and $700{ }^{\circ} \mathrm{C}$, respectively, and these values are very similar with those of T91 (Sawada et al., 2007). Zielinski et al. (2016) reported that the Z-phase is hardly found in thermally aged specimens of P92 (X10CrWMoVNb9-2) at 600 and $650{ }^{\circ} \mathrm{C}$ for 100000 $\mathrm{h}$, but they also highlighted that the MX particles with high $\mathrm{Cr}$ content were observed when aged at $600{ }^{\circ} \mathrm{C}$ for 70 $000 \mathrm{~h}$, and so these particles may be the Z-phase. Their report is significantly different from the observations on the Z-phase formation in Grade 92 in previous work (Haetterstrand \& Andren, 2001; Sawada et al., 2006; 2007; Danielsen, 2007; Yoshizawa et al., 2009). It must be noted that the heat treatment conditions of the Zielinski's samples were unknown and the sample contains $0.009 \%$ boron, which was analyzed by themselves. However, this value is out of the specification range (B: $0.001-0.006 \%$ ) of ASME SA-213/SA-213M and PN-EN 10216-2 (European standard in Poland for P92). Although the effect of B on the formability of the Z-phase is not yet to be clarified, it would not be unreasonable to suppose that the Z-phase is less easily formed in boron containing steel, Grade 92 compared to Grade 91. The reason is as follows: The addition of boron tends to suppress the growth rate of MX in high Cr martensitic steel (Azuma et al., 2002), which results in stabilization of MX and so, MX particles stabilized by boron may not easily decompose and thus would not easily transform into the Z-phase. It was previously reported that $\mathrm{Cr}, \mathrm{Ni}$, and Co promote, but that $\mathrm{C}$ suppresses the formation of the Z-phase (Danielsen \& Hald, 2009; Yan et al., 2013). However, the effect of micro-alloying elements on the formation of the Z-phase in Grade 92 is insufficiently studied.

Usually, BN particles that are $1-2 \mu \mathrm{m}$ in size are observed as normalized Grade 92 (Gu, West, \& Thomson, 2014). Cavities are formed on the interfaces surrounding the large $\mathrm{BN}, \mathrm{MnS}$, and $\mathrm{Al}_{2} \mathrm{O}_{3}$ particles, which cause a decrease in creep ductility and thus affects rupture lives (Parker \& Siefer, 2018). Massive Laves phase particles also cause the formation of cavities (Lee, Armaki, Maruyama, Muraki, \& Asahi, 2006). Reduction of area $(R A)$ falls zero in some long-term rupture tests of Grade 92. However, this is an apparent phenomenon caused by a number of cavities stored inside the specimens, because the rupture appearance is ductile and the moderate elongation at rupture is still confirmed (Abe, Ohba, Miyazaki, Toda, \& Tabuchi, 2018).

\section{Results of Analysis}

\subsection{Cr-1 W Steel}

\subsubsection{Time to Rupture and Analysis of Creep Curves}

The relationship between stress and time to rupture of $9 \mathrm{Cr}-1 \mathrm{~W}$ steel was obtained at $550-650{ }^{\circ} \mathrm{C}$ (Abe et al., 1992). The data were classified into three groups, Grs.I-III, and the regression lines were obtained using Equation 3 at each temperature in each group. The results are shown in Figure 2. In the classification, stress vs. time to a specific strain, $t_{\varepsilon}$, diagrams were obtained using the original creep curves and were referred, where $\varepsilon=0.25-$ $10 \%$. The typical diagrams are shown in Figure A2. In Figure A2a), $t_{0.25}$ denotes the time to $0.25 \%$ strain and similar symbols are used in this paper. Linear relationships between linear stress and logarithmic time are confirmed in Figure A2 similar to Figure 2, which indicates that the time to a specific strain can be applied to Equation 1 if we replace $t_{r}$ with $t_{\varepsilon}$. Figure 2 shows that the lower the stress, the longer the $t_{r}$ at constant temperature or the $\sigma-t_{r}$ relationship changes to an inverse J-shape from Gr.I to Gr.III at $600{ }^{\circ} \mathrm{C}$. This trend is similar in the $\sigma-t_{\varepsilon}$ relationships for $\varepsilon=1-10 \%$. However, with respect to the $\sigma-t_{1}$ relationship, there is 
slight difference between Gr.I and Gr.II, conversely, $t_{0.25}$ for $G r . I I$ is shorter than that estimated from the data for Gr.I, which we refer to as the Z-shape (Figure A2a)).

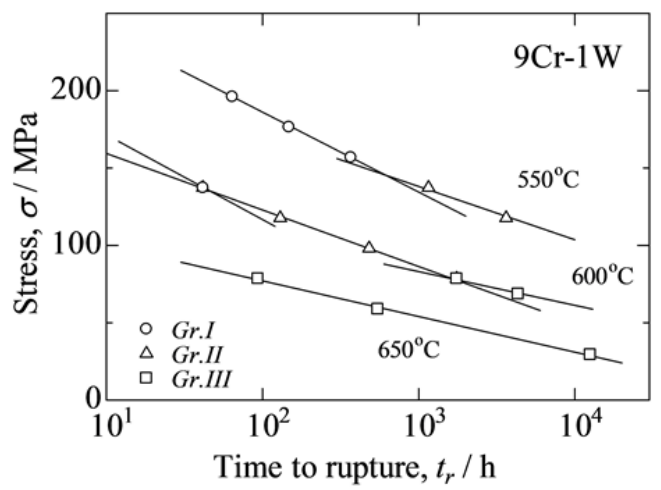

Figure 2. Relationship between stress and time to rupture for $9 \mathrm{Cr}-1 \mathrm{~W}$ steel. Regression lines for each data group are explained in Section 4.1.1 and the data were analyzed employing the exponential method explained in

Section 2 (Similar explanation is omitted, hereinafter)

The inverse J-shape relationship in a $\sigma-t_{\varepsilon}$ diagram is frequently observed in high temperature creep of metals, and the inverse J-shape relationship is of course confirmed in the double logarithmic plot of $\sigma$ and $t_{r}$, that is usually used. In such cases, the creep data are plotted in a double logarithmic diagram of $\sinh (\alpha \sigma)$ and $t_{r}$, where $\alpha$ is a parameter to obtain a linear relationship at a temperature in the diagram (Garofalo, 1963). However, there is no physical meaning in an adjustable parameter, $\alpha$ and thus we cannot infer what happens in a wide range of times to rupture. On the other hand, Equation 1 is originally introduced as an approximate equation for high stresses of a theoretical equation, $\dot{\varepsilon} \propto \sinh \sigma$, proposed for a thermally activated process applied to a dislocation motion (Schoeck, 1980). Equation 1 holds when $X=\sigma V / R T>1$, and we obtain the relationship $X \sim n$ based on an extremely rough approximation, where $n$ is the well-known stress exponent in a power law (Tamura et al., 2003). But, as a matter of course, the physical meaning of $X \sim n$ cannot be solved. We obtain $\sigma>9.8 \mathrm{MPa}$ for $X>1$ by substituting the average temperature and the value of $V$ for Gr.III. The minimum stress for Gr.III is $29.4 \mathrm{MPa}$ and is much larger than the critical value of $9.8 \mathrm{MPa}$, and thus, Equation 1 can be successfully applied to Gr.III for $9 \mathrm{Cr}-1 \mathrm{~W}$ steel. Therefore, the phenomenon that the time to rupture for $9 \mathrm{Cr}-1 \mathrm{~W}$ steel under lower stresses are prolonged when compared to those estimated from the data under higher stresses and lower temperatures should be able to be explained metallurgically.

Figure 3 shows the relationship between strain and the average normalized strain rate (NSR) plotted in a double logarithmic diagram, where the strain rate at a specific strain is divided by each $M C R$ and then the normalized $N S R \mathrm{~s}$ are averaged for each group. In the figure, the strain in the far right for each group is the average of the $E L$ (the same, hereafter). In Figure 3, characteristics of each group are clearly shown, namely, the average NSRs for Gr.II at an early stage of transition creep are rather high compared to those of Gr.I followed by rapid hardening (the slope, $\Delta \log ($ average $N S R) / \Delta \log \varepsilon$, is steep), and this trend becomes more distinct for Gr.III. Strains for the $M C R$ s for each group in Figure 3, range between $2-5 \%$. In accelerating creep, the average NSRs for Gr.II are rather small, but the slopes are all the same. It is thought from Figure 3 that the rupture behavior of $1 \mathrm{~W}$ steel shown in Figure 2 is related to the average NSRs not only in accelerating creep but also in transient creep as shown in Figure 3.

Figure 4 shows the variations of $Q V C$ as functions of strain for each group. Strains to the $M C R$ and the maximum value in the vertical axis are marked by a solid and a large mark, respectively, for each group (the same for all similar figures, but the explanation is omitted hereinafter). The values of $Q$ at rupture for all groups in Figure 4 are approximately $400 \mathrm{kJmol}^{-1}$ and are little larger than the activation energy for self-diffusion of alpha-iron (Oikawa, 1982). Moreover, the values of $C$ are $\sim 20$, which are the same values confirmed in many heat-resistant steels (Tamura et al., 2013). Therefore, although the microstructure before testing of $1 \mathrm{~W}$ steel is tempered martensite, the steels are judged to be ruptured in a fully recovered state. The variations of $Q V C$ as functions of strain for each group shown in Figure 4 are considered to be statistically significant referring to the previous work (Tamura \& Abe, 2015b). 


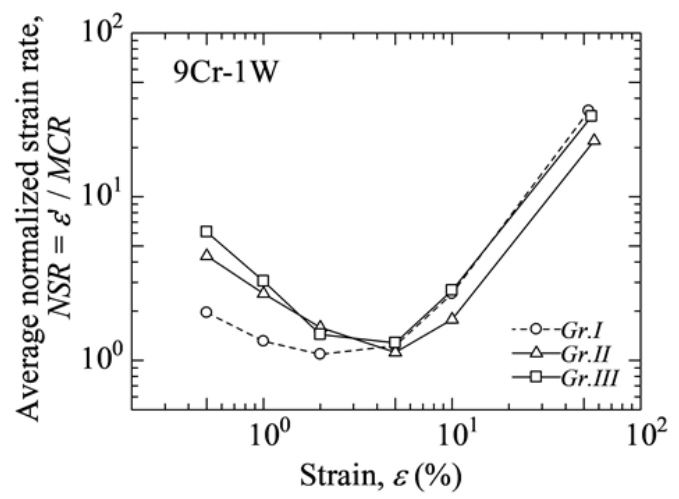

Figure 3. Average NSR vs. strain relationship for $9 \mathrm{Cr}-1 \mathrm{~W}$ steel

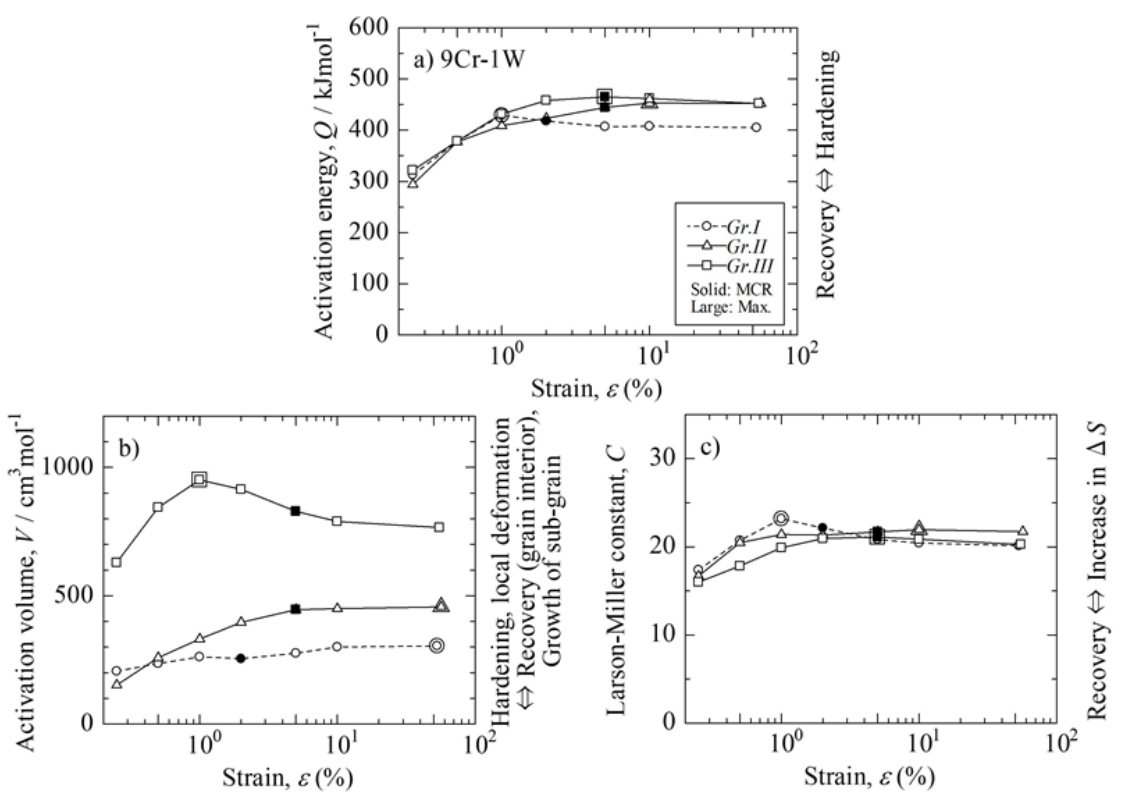

Figure 4. The results of the $Q V C$ analysis are shown as functions of creep strain for each group of $9 \mathrm{Cr}-1 \mathrm{~W}$ steel: a) the activation energy, b) the activation volume, and c) the Larson-Miller constant. The values for $M C R$ and the maximum values are marked for each group by solid and large marks, respectively. On the right side of each figures the guide lines for the metallurgical reactions corresponding to the increase and decrease of each variable, $Q V C$, are indicated (the same for the similar diagrams, hereafter)

\subsubsection{Gr.I}

$Q$ and $C$ shown in Figure 4 increase up to a strain of $1 \%$ in a transient creep range, and then tend to decrease slightly with increasing strain. The increase in $Q$ denotes strain hardening and the increase in $C$ denotes the increase in entropy associated with the movement of the dislocations. $V$ tends to increase slightly in throughout the whole duration of creep, which indicates that recovery inside the sub-grains occurs continuously. The observations of the microstructure of this steel (Abe et al., 1991; 2003; 2005) indicated that the dislocation density inside the sub-grains decreases during creep and no precipitates except for $\mathrm{M}_{23} \mathrm{C}_{6}$ form under the average conditions of Gr.I, i.e., $563{ }^{\circ} \mathrm{C}, 167 \mathrm{MPa}$, and $t_{r}=155 \mathrm{~h}$. In this study, each term corresponding to a sub-grain denotes a minimum unit of creep deformation, which indicates the martensite lath, block, packet, the sub-grains themselves, or in some cases the heterogeneously recovered and deformed zone explained in the later sections, 4.1.4 and 4.1.5.

In a strain range of up to $1 \%$ strain, hardening, i.e., the increase in $Q$, and recovery, i.e., the simultaneous increase in $V$, are confirmed. However, these are not contradictory statements. This occurs because the increase in $V$ only 
denotes recovery inside the sub-grains, and consequently the dislocations that are swept out of the sub-grains to the PAGBs and boundaries of packet, block, and lath martensite during creep, are accumulated. However, these accumulated dislocations cannot move or be annihilated immediately, because many nearby sub-grains jostle and do not deform each other freely. We refer to this phenomenon as Sub-grain Boundary Strengthening by Dislocations (SBSD). Essentially, SBSD is explained as follows: Boundaries are strengthened due to the dislocations caused by the recovery inside the sub-grains, which results in an increase in $Q$, and consequently the back stress to the dislocation increases, the effective stress as well as the dislocation velocity decreases, and therefore, the creep rate at an early stage of creep decreases with increasing strain, as shown in Figure 3. Martensitic steel contains many dislocations, and therefore SBSD can easily occur compared with other soft materials. Therefore, SBSD is always observed in an early stage of transient creep and is an essential hardening mechanism for martensitic steel. After $M C R$ at $2 \%$ strain, $Q$ and $C$ tend to decrease and $V$ increases with an increase in strain, and so, accelerating creep is in progress recovering microstructure. Although transient creep still continues in a strain range of $1-2 \%$, both $Q$ and $C$ decrease, which reminds us that recovery may begin from $1 \%$ strain. However, $V$ also slightly decreases, and therefore, another mechanism than that occurring in an accelerating creep range should occur in a strain range of $1-2 \%$. The phenomenon of a decrease in $V$ in a transition creep range is more clearly observed in Gr.III and therefore this phenomenon will be explained in detail in Sections 4.1.4 and 4.1.5.

\subsubsection{Gr.II}

Both $Q$ and $V$ increase up to $5 \%$ strain, near the $M C R$ strain, and at higher strains both $Q$ and $V$ are roughly constant. On the other hand, $C$ increases rapidly up to $1 \%$ strain and then remains flat. Therefore, SBSD occurs rightly in Gr.II up to $1 \%$ strain like in Gr.I, but both $Q$ and $V$ still continue to increase until higher strains than $\varepsilon=1 \%$, where the $Q$ for Gr.I begins to decrease. Coarsening of $\mathrm{M}_{23} \mathrm{C}_{6}$ occurs in Gr.II (Abe et al., 2003), because the average test conditions of $G r . I I$ are $583{ }^{\circ} \mathrm{C}, 114 \mathrm{MPa}$, and $t_{r}=1198 \mathrm{~h}$ and the average temperature is higher than that in Gr.I. It is generally accepted that the dislocation density inside sub-grains decreases and the sub-grain size increases with increasing creep strain (Abe et al., 1992; Sawada et al., 2011). In these cases, some of the $\mathrm{M}_{23} \mathrm{C}_{6}$ particles precipitated on the sub-grain boundaries temporarily dissolve into the matrix accompanying the migration of the sub-boundaries during the growth of sub-grains and then finer particles of $\mathrm{M}_{23} \mathrm{C}_{6}$ re-precipitate on the newly migrated boundaries (Abe et al., 1991). We call this phenomenon Dissolving and Finely Re-Precipitation (DFRP). If this mechanism did not operate for Gr.II, $Q$ would decrease continuously after SBSD like Gr.I or the strong SBSD could continue through an accelerating creep range, but that is not actually true. As a consequence, the increases in both $Q$ due to fine precipitates and $V$ due to the growth of sub-grains continue up to higher creep strains than those in Gr.I leading to increase in $t_{\varepsilon}$ and $t_{r}$ of Gr.II as estimated from that of Gr.I (Figures 2 and $\mathrm{A} 2 \mathrm{~b}$ and $\mathrm{c}$ )).

\subsubsection{Gr.III}

$Q$ increases largely up to $2 \%$ strain and then decreases slightly from $5 \%$ strain. $C$ changes similarly to $Q$. On the other hand, $V$ increases largely up to $1 \%$ strain and then clearly decreases up to $10 \%$ strain, followed by a slight decrease up to rupture. SBSD occurs up to $1 \%$ strain like Grs.I and II. The DFRP mechanism for $\mathrm{M}_{23} \mathrm{C}_{6}$ caused by the growth of sub-grains is considered to operate remarkably in $\varepsilon=1-5 \%$ as compared to Gr.II. The reason for this is as follows: Since the average test conditions of $\mathrm{Gr} . I I I\left(630^{\circ} \mathrm{C}, 62.8 \mathrm{MPa}\right.$, and $\left.t_{r}=3857 \mathrm{~h}\right)$ are positioned in a higher temperature and longer time range than those for $G r . I I$, the DFRP mechanism for $\mathrm{M}_{23} \mathrm{C}_{6}$ begins to start at a smaller strain than that for Gr.II. The values of $C$ in Gr.III are smaller than those of Gr.II, which indicates that the microstructure of Gr.III is more progressively recovered than that of Gr.II.

The values of $V$ are considerably high over the entire range of creep strain. Therefore, there should be considerable recovery within the sub-grains. On the other hand, $V$ decreases distinctly after the peak at $1 \%$ strain; hence, an inverse phenomenon against recovery is imperative. However, the precipitation hardening that explains the decrease in $V$ should never occur inside the sub-grains of $9 \mathrm{Cr}-1 \mathrm{~W}$ steel. Therefore, the decrease in $V$ at strains higher than $1 \%$ must be caused by heterogeneous creep deformation like the creep phenomena for welded joints explained in Section 2.5. When SBSD reaches a peak at 1\% strain, the recovered zones or sub-grains are newly created near the weakest boundaries including PAGBs accidentally formed by the SBSD phenomenon, followed by strain- and stress concentration and the consequent stress relaxation due to heterogeneous deformation. These sequential phenomena should usually occur immediately after the final stage of SBSD, an initial creep deformation mechanism for martensitic steel is explained in Section 4.1.2. We call this series process Heterogeneous Recovery and Heterogeneous Deformation (HRHD). 
If we imagine that a simple local deformation occurs continuously after $1 \%$ strain, accelerating creep may take place. However, as seen in Figure 4a), $Q$ clearly increases up to $\varepsilon=5 \%$ and consequently the strain rate decreases as seen in Figure 3. Therefore, during the occurrence of HRHD at strains higher than 1\%, the DFRP mechanism for $\mathrm{M}_{23} \mathrm{C}_{6}$ takes place continuously and actively, and so, $Q$ of Gr.III becomes higher than that of Gr.II as seen in Figure $4 \mathrm{a}$. This mechanism decreases the strain rate and as a result, the $t_{\varepsilon}$ and $t_{r}$ are elongated compared to those of Gr.II. Additionally, $V$ continues to decrease after $5 \%$ strain, but the rate of decrease declines. This phenomenon may be interpreted in two ways: First, the degree of HRHD essentially slows down, and second, the increase in $V$ due to the growth of sub-grains usually occurs during creep. Metallurgical observations are needed to explain the decrease in $V$ due to HRHD.

\subsubsection{SBSD and HRHD}

In the previous sections, 4.1.2 - 4.1.4, it was explained that the decrease in the creep rate during the early stage of transient creep of $9 \mathrm{Cr}-1 \mathrm{~W}$ martensitic steel is caused by SBSD and that the decrease in the creep rate during the later stage of transient creep for $G r$.III after the peak of $V$, is caused by HRHD accompanying the DFRP for $\mathrm{M}_{23} \mathrm{C}_{6}$. Both SBSD and the subsequently occurring HRHD are new concepts, respectively and therefore, the validities of these concepts must be verified by further investigations.

In the above analyses, $Q V C$ are obtained using $t_{r}$ and $t_{\varepsilon}$ and not the strain rates at a specific strain. The reason for this is that the rupture data are most popular and the number of $t_{r}$ accumulated is very large, though the creep rates near rupture calculated using Equation 8 are significantly affected by necking of the specimen. However, the strain rate is an important parameter which adequately reflects the state of the microstructure at a given strain. Therefore, for the detailed analysis of both SBSD and HRHD which are observed in transient creep of Gr.III of $9 \mathrm{Cr}-1 \mathrm{~W}$ steel, the activation energy, $Q_{s}$, the activation volume, $V_{s}$, and the Larson-Miller constant, $C_{s}$, are re-evaluated using the strain rates at a specific strain calculated using Equation 8, instead of $t_{r}$ and $t_{\varepsilon}$. The following equation similar to Equation 3 obtained from Equations 1, 2, and 6 and the regression analyses were made.

$$
\log \dot{\varepsilon}=-\left[Q_{s}\right]+\left[V_{s}\right]+C_{s}
$$

where the square brackets denote the same as Equation 3. The results of $Q_{s}, V_{s}$, and $C_{s}$ for $G r . I I I$ of $9 \mathrm{Cr}-1 \mathrm{~W}$ steel are shown as functions of creep strain in Figure 5. The variations in $Q_{s}, V_{s}$, and $C_{s}$ are very similar with those shown in Figure 4 for Gr.III. The values of $Q_{s}, V_{s}$, and $C_{s}$ in Figure 5 are $0.81-1.26$ times of those of $Q V C$ in Figure 4. Analyses were made for all data including a creep rate at rupture, but the values of $Q_{s}, V_{s}$, and $C_{s}$ at rupture are not thoroughly discussed hereafter in the similar figures shown later, because the creep rate at rupture is affected by necking of the specimen. The $Q_{s}, V_{s}$, and $C_{s}$ shown in Figure 5 all increase up to $1 \%$ strain and therefore, the occurrence of SBSD is reconfirmed. For strain in the range of $1-5 \%$ of transient creep, although the rates of increase in both $Q_{s}$ and $C_{s}$ decline, both the $Q_{s}$ and $C_{s}$ still increase and the $V_{s}$ maintains a high level. Therefore, the SBSD phenomena are considered to continue to an $M C R$ point. However, since the $V_{S}$ decreases within the strain range of $1-5 \%$, an additive reaction other than the SBSD must be occurring. To account for this observation, the relationship between the changes in $Q_{s}, V_{s}$, and $C_{s}$ with increasing strain and the changes in the corresponding strain rate was analyzed. Table 4 shows the differences in the $\left[Q_{s}\right],\left[V_{s}\right], C_{s}$, and $\log \dot{\varepsilon}$ in Equation 11 between two adjacent strains, $\Delta\left[Q_{s}\right], \Delta\left[V_{s}\right], \Delta C_{s}$, and $\Delta \log \dot{\varepsilon}$ at an average temperature and under an average stress for Gr.III of $9 \mathrm{Cr}-1 \mathrm{~W}$ steel. Negative values for $\Delta\left[Q_{s}\right]$ are listed in Table 4 to set the summation of the above three parameters in each column equal to $\Delta \log \dot{\varepsilon}$ and the determinant terms for the strain rate are indicated in bold-italics. By exclusively considering the changes in $Q_{s}$, Table 4 suggests that the changes in $\Delta \log \dot{\varepsilon}$ can be explained by the changes in $\Delta\left[Q_{s}\right]$, i.e., the magnitude of resistance to the mobile dislocations, through the whole creep strain. As seen in Table $4,-\Delta\left[Q_{s}\right]<0$ is really a determinant term in $\varepsilon=1-5 \%$, but with only that the change in strain rate does not become negative or transient creep does not hold. The fact of $\Delta\left[V_{s}\right]<0$ is an absolute necessity for transient creep to occur within the strain range of $1-5 \%$. In other words, if we bear in mind that $-\Delta\left[Q_{s}\right]<0$ is also a determinant term and that $Q$ for Gr.III is larger than that for Gr.I as shown in Figure 4, it can be understood that the sequential processes of heterogeneous recovery and heterogeneous deformation, i.e., HRHD, followed by both the creation of new sub-grains and the DFRP mechanism operating on $\mathrm{M}_{23} \mathrm{C}_{6}$, as mentioned in Section 4.1.3, are an absolutely necessary packaged-process for transient creep in the strain range of $1-5 \%$ for $G r . I I I$ of $9 \mathrm{Cr}-1 \mathrm{~W}$ steel.

When new and small sub-grains are created by heterogeneous recovery, the average migration distance or movable area for dislocations may decrease locally, which causes a decrease in $V$. However, recovery essentially continues during creep, and so, the newly born sub-grains grow easily, i.e., $V$ increases soon, if there are no pinning obstacles against grain growth. However, Figure 5 shows not only the decrease in $V_{S}$, but $Q_{S}$ also 
increases within $\varepsilon=1-5 \%$. This suggests that when the DFRP mechanism should operate on $\mathrm{M}_{23} \mathrm{C}_{6}$ particles, prevention of excess growth of the newly created sub-grains occurs. This means that even though the HRHD occurs within this strain range, clear hardening that resists the softening effect due to HRHD occurs by the DFRP mechanism. This is the origin of the increase in $Q_{s}$ within $\varepsilon=1-5 \%$ for Gr.III of $1 \mathrm{~W}$ steel despite the occurrence of the HRHD. In the above situation, we must remind that the $V$ for the welded joint is smaller than that of the base metal. Thus, we can assume that heterogeneous deformation would occur in the martensitic steel when $V$ decreases. However, we did not observe the occurrence of macroscopic local deformation like welded joints. Instead, we confirmed that the size of heterogeneous recovery is, at most, $10 \mu \mathrm{m}$, which is the size of the primary austenite grains (Kushima et al., 1999). The recovered areas randomly appear near the weak boundaries, with less SBSD through a specimen. Therefore, the specimen deforms uniformly on a macroscopic level.

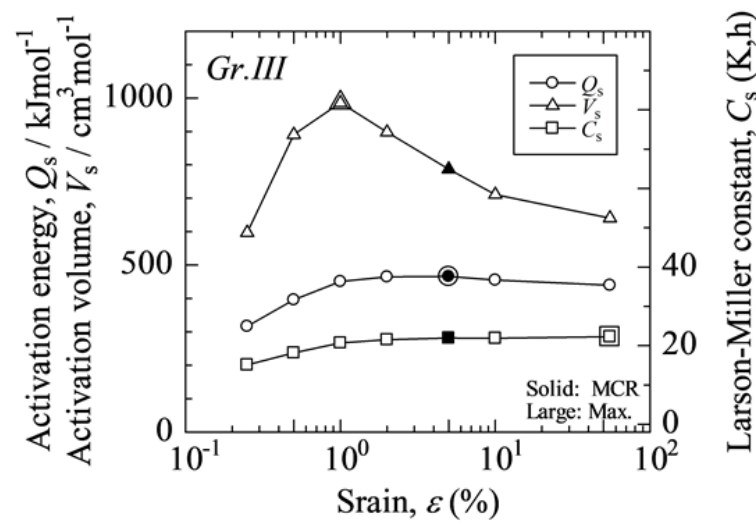

Figure 5. $Q_{s}, V_{s}$, and $C_{s}$ for $G r . I I I$ of $9 \mathrm{Cr}-1 \mathrm{~W}$ steel are shown as functions of creep strain

By the way, an early stage of transient creep of martensitic steel is frequently explained by another proposed mechanism apart from SBSD. There are many excess dislocations inside a sub-grain, the dislocation density inside the sub-grain decreases as creep strain progresses, and hence the strain rate decreases according to the Orowan equation (Equation 5). This explanation is qualitatively correct, however a quantitative examination is necessary. It is rare that the dislocation density inside a sub-grain decreases by one order of magnitude within a short range of strain, but assuming that the dislocation density inside sub-grains decreases by one order of magnitude up to $1 \%$ strain, then the dislocation density due to the dislocations that are swept out increases by one order of magnitude, and in this case, the value of $C$ or the first term in Equation 9 increases only by one. However, Figures 4 shows that the evaluated values of $C$ increase by at least 3.9 for Gr.III through the increase in creep strain from $0.25 \%$ to $1 \%$ and therefore, the explanation that the decrease in the strain rate of the martensitic steel is caused by the decrease in excess dislocations inside sub-grains is considered to be insufficient to account for the observed increases in $C$. The decrease in strain rate in an early stage of creep can be preferentially explained based on the fact that both the second term of Equation 9, i.e., the entropy term, and an increase in $Q_{s}$ during this strain range. As seen in Table 4, both $\Delta Q_{s}$ and $\Delta C_{s}$ increase significantly up to $1 \%$ strain. The increase in $Q_{s}$ represents the increase in the back stress against the motion of dislocations caused by the accumulated dislocations that are swept out of the sub-grains on the sub-boundaries, whereas the increase in $C_{S}$ is mainly caused by the increase in entropy associated with the dislocation movement. Table 4 also shows that $V_{s}$ increases largely up to $1 \%$ strain, which denotes that many dislocations are swept out of the sub-grains and so the movable area of dislocations increases. These observations support the concept of SBSD explained in Section 4.1.2.

In Section 4.1.2, we mentioned that both $Q$ and $C$ also decrease in $\varepsilon=1-2 \%$ for Gr.I where $V$ decreases. This situation differs from the case for Gr.III although $V$ decreases in both groups. Therefore, we performed the $Q_{s} V_{s} C_{s}$ analyses similar to those shown in Table 4 on the data of $\varepsilon=1-2 \%$ of Gr.I. It was confirmed from the analyses that the determinant terms to reduce the strain rate are the decreases in both $\Delta\left[V_{s}\right]$ and $\Delta C_{s}$, though the Table is omitted for simplicity. Namely, HRHD is also necessary to reduce creep rate in $\varepsilon=1-2 \%$ for Gr.I of $1 \mathrm{~W}$ steel. 
Table 4. The differences in $Q_{s}, V_{s}, C_{s}$, and $\log (\dot{\varepsilon})$ between two adjacent strains at an average temperature and under an average stress for $G r$.III of $9 \mathrm{Cr}-1 \mathrm{~W}$ steel

\begin{tabular}{ccccccc}
\hline Strain range & {$[0.25-0.5 \%]$} & {$[0.5-1 \%]$} & {$[1-2 \%]$} & {$[2-5 \%]$} & {$[5-10 \%]$} & {$\left[10 \%-t_{r}\right]$} \\
\hline$-\Delta\left[Q_{s}\right]$ & -4.59 & $-\mathbf{3 . 1 8}$ & $\mathbf{- 0 . 8 0}$ & $\mathbf{- 0 . 0 8}$ & $\mathbf{0 . 6 3}$ & $\mathbf{0 . 9 0}$ \\
$\Delta\left[V_{S}\right]$ & 1.07 & 0.35 & $\mathbf{- 0 . 3 2}$ & $\mathbf{- 0 . 4 0}$ & -0.28 & -0.26 \\
$\Delta C_{S}$ & 3.07 & 2.53 & 0.82 & 0.42 & -0.06 & 0.39 \\
$\Delta \log (\dot{\varepsilon}, \% / \mathrm{h})$ & -0.45 & -0.30 & -0.30 & -0.06 & 0.30 & 1.03 \\
\hline
\end{tabular}

Table 5. Summary for the operation of SBSD, DFRP, and HRMD as functions of creep stages for each data group of $9 \mathrm{Cr}-1 \mathrm{~W}$ steel. $Q_{s+}$ etc. and $Q_{s-}$ etc. denote an increase and a decrease, respectively, for indicated creep stages. Determinant terms in each creep range are shown in bold-italics. Parentheses denote phenomena are weak

\begin{tabular}{|c|c|c|c|}
\hline \multirow{2}{*}{$\begin{array}{l}\text { Grs. and average } \\
\text { temperature }\end{array}$} & \multicolumn{2}{|c|}{ Transient creep } & \multirow{2}{*}{$\frac{\text { Accelerating creep }}{M C R-10 \%}$} \\
\hline & $\varepsilon<1 \%$ & $1 \%-M C R$ & \\
\hline $\begin{array}{c}G r . I \\
563{ }^{\circ} \mathrm{C}\end{array}$ & $\begin{array}{l}\boldsymbol{Q}_{s+}, \mathrm{V}_{s+}, \mathrm{C}_{\mathrm{s}+} \\
\quad \mathrm{SBSD}\end{array}$ & $\begin{array}{c}\mathrm{Q}_{s^{-}}, \boldsymbol{V}_{\boldsymbol{s}_{-}}, \boldsymbol{C}_{\boldsymbol{s}-} \\
\text { HRHD, (Recovery) }\end{array}$ & $\begin{array}{c}\boldsymbol{Q}_{s^{-}}, \boldsymbol{V}_{\boldsymbol{s}^{+}}, \mathrm{C}_{\mathrm{s}^{-}} \text {and then } \mathrm{Q}_{\mathrm{s}^{-}}, \boldsymbol{V}_{\boldsymbol{s}^{+}}, \boldsymbol{C}_{\boldsymbol{s}+} \\
\text { Recovery }\end{array}$ \\
\hline $\begin{array}{c}G r . I I \\
583^{\circ} \mathrm{C}\end{array}$ & $\begin{array}{l}\boldsymbol{Q}_{s+}, \mathrm{V}_{s+}, \mathrm{C}_{\mathrm{s}+} \\
\quad \mathrm{SBSD}\end{array}$ & $\begin{array}{c}\boldsymbol{Q}_{\boldsymbol{s +}}, \mathrm{V}_{\boldsymbol{s +}}, \boldsymbol{C}_{\boldsymbol{s +}} \\
\text { Recovery, DFRP for } \mathrm{M}_{23} \mathrm{C}_{6}\end{array}$ & $\begin{array}{c}\left(\mathrm{Q}_{\mathrm{s}+}, \mathrm{V}_{s-}\right), \boldsymbol{C}_{\boldsymbol{s}+} \\
\text { (Recovery), DFRP for } \mathrm{M}_{23} \mathrm{C}_{6}\end{array}$ \\
\hline $\begin{array}{l}G r . I I I \\
630^{\circ} \mathrm{C}\end{array}$ & $\begin{array}{l}\boldsymbol{Q}_{s_{+}}, \mathrm{V}_{s_{+}}, \mathrm{C}_{\mathrm{s+}} \\
\quad \mathrm{SBSD}\end{array}$ & $\begin{array}{c}\boldsymbol{Q}_{\boldsymbol{s}_{+}}, \boldsymbol{V}_{\boldsymbol{s}^{-}}, \mathrm{C}_{\mathrm{s}^{+}} \\
\text {HRHD, DFRP for } \mathrm{M}_{23} \mathrm{C}_{6}\end{array}$ & $\begin{array}{c}\boldsymbol{Q}_{\boldsymbol{s}_{-}}, \mathrm{V}_{s^{-}}, \mathrm{C}_{\mathrm{s}^{-}} \\
(\mathrm{HRHD}), \mathrm{DFRP} \text { for } \mathrm{M}_{23} \mathrm{C}_{6}\end{array}$ \\
\hline
\end{tabular}

Table 5 summarizes for convenience in what conditions SBSD, DFRP, and HRMD occur based on the above discussion and the $Q_{s} V_{S} C_{S}$ analyses for $9 \mathrm{Cr}-1 \mathrm{~W}$ steel. It is found from the above that SBSD is an essential creep process at the beginning of creep of martensitic steel. In a later stage of transient creep, from $1 \%$ strain to an $M C R$ point, though recovery is the basic process for martensitic steel, the decrease in creep rate is maintained through the combination of processes such as a decrease in the entropy term, $C_{s}$, hardening due to DFRP of $\mathrm{M}_{23} \mathrm{C}_{6}$, or HRHD. In accelerating creep, the decrease in $Q_{s}$ due to recovery is the determinant term, but when $Q_{s}$ does not decrease so largely with an increase in strain for example due to DFRP for $\mathrm{M}_{23} \mathrm{C}_{6}$, an increase in $C_{s}$ is the determinant term (Grs.I and II). Both SBSD and HRHD are also frequently observed in transient creep of the strengthened martensitic steels, as mentioned in the later sections for Grades 91 and 92 , though both $Q$ and $C$ decrease.

\subsubsection{Factors for the Increase in Strength of Gr.III as Compared to Those of Gr. II}

HRHD denotes a series of multiple processes approaching to creep rupture without any hardening process. However, we confirmed from the above that $t_{\varepsilon}$ and $t_{r}$ in Gr.III are longer than those estimated from Gr.II for all strains and that HRHD definitely occurs in Gr.III but apparently does not occur in Gr.II. Therefore, to investigate the relationship between this fact and metallurgical reactions, $t_{\varepsilon}$ and $t_{r}$ for Grs.II and III under the conditions of the average temperature and stress of Gr.III were calculated (these variables are symbolized as $t_{I I}$ and $t_{I I I}$, respectively), $[Q],[V]$, and $C$ were calculated according to Equation 3, and the differences between the obtained values of $[Q],[V]$, and $C,(\Delta[Q], \Delta[V]$, and $\Delta C)$ were also calculated. The results for each strain are shown in Table 6. In the table, the differences between $\operatorname{logarithmic} t_{I I I}$ and $t_{I I}$, i.e., $\log \left(t_{I I I} / t_{I I}\right)$, and key factors for the differences are also shown. In Table 6 , the values of $-\Delta[V]$ and $-\Delta C$ are listed so that the summation of each term becomes $\log \left(t_{I I I} / t_{I I}\right)$ and moreover, the determinant terms for $\log t_{I I I}>\log t_{I I}$ are bold-italicized. It is found from Table 6 that the increase in strength for Gr.III compared to the strength estimated from Gr.II is caused mainly by the recovery due to the decrease in $\Delta C$ except for $2 \%$ strain. However, Table 6 clearly shows that the increase in $\Delta[Q]$ is also key factor during creep from 0.25 to $10 \%$ strain (except for $0.5 \%$ strain) for the increase in strength of Gr.III, $\log t_{I I I}>\log t_{I I}$, where the DFRP mechanism for $\mathrm{M}_{23} \mathrm{C}_{6}$ is operating. Therefore, this suggests that retaining the refinement and stabilization of $\mathrm{M}_{23} \mathrm{C}_{6}$ during creep is considered to be very important for the development of heat-resistant steel. 
Table 6. Key factors determining increase in strength for $G r . I I I$ of $1 \mathrm{~W}$ steel, $\log \left(t_{I I I} / t_{I I}\right)$, for each creep strain

\begin{tabular}{ccccccccc}
\hline Strain $(\%)$ & 0.25 & 0.5 & 1 & 2 & 5 & 10 & 45.2 \\
\hline$\Delta[Q]$ & $\mathbf{1 . 5 9}$ & 0.10 & $\mathbf{1 . 3 2}$ & $\mathbf{2 . 0 1}$ & $\mathbf{1 . 1 9}$ & $\mathbf{0 . 5 1}$ & -0.01 \\
$-\Delta[V]$ & -1.74 & -2.14 & -2.26 & -1.89 & -1.40 & -1.24 & -1.13 \\
$-\Delta C$ & $\mathbf{0 . 6 9}$ & 2.64 & $\mathbf{1 . 5 1}$ & 0.39 & $\mathbf{0 . 5 9}$ & $\mathbf{1 . 0 8}$ & $\mathbf{1 . 4 6}$ \\
$\log \left(t_{I I I} / t_{I I}\right)$ & 0.53 & 0.60 & 0.57 & 0.51 & 0.38 & 0.35 & 0.32 \\
& Grain boundary hardening & Recovery due to $\Delta C<0$ & DFRP for $\mathrm{M}_{23} \mathrm{C}_{6}$ & & Recovery due to $\Delta C<0$ \\
\hline
\end{tabular}

\subsubsection{Schematic Illustration for Changes in Microstructure during Creep of 9C-1W Steel}

The changes in microstructure during creep for Grs.I - III of 9Cr-1W steel are illustrated in Figure 6 based on the discussion on the changes in the $Q V C$ s of $1 \mathrm{~W}$ steel during creep. Two matters are highlighted, namely that the grain boundary-hardening caused by the SBSD mechanism occurs at the beginning of creep and that HRHD occurs immediately after SBSD during transient creep. Further, in Grs.II and III the DFRP mechanism is operating in $\mathrm{M}_{23} \mathrm{C}_{6}$ and the growth of the pre-existing sub-grains or newly created sub-grains associated with HRHD are prevented. In the figure, pre-existing $\mathrm{M}_{23} \mathrm{C}_{6}$ particles are omitted for simplicity.

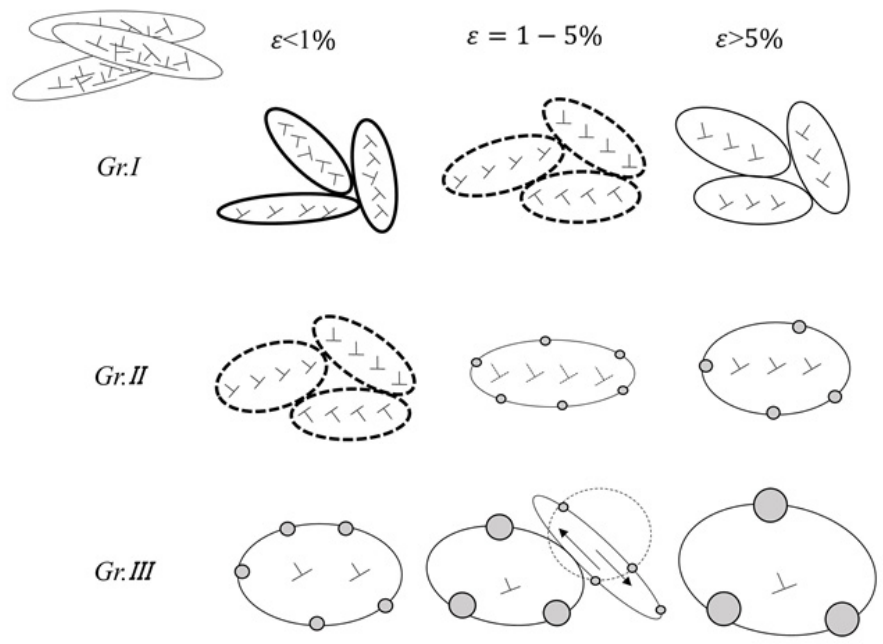

Figure 6. Schematic illustration of changes in microstructure during creep of 9Cr-1W steel. Gr.I: SBSD (bold line) for $\varepsilon<1 \%$ followed by weak HRHD in transient creep and then, general recovery in accelerating creep. Gr.II: SBSD followed by the growth of sub-grains and the DFRP mechanism for $\mathrm{M}_{23} \mathrm{C}_{6}$ on the newly migrated boundaries. Gr.III: Compaction of deformation processes of Gr.II within a short strain followed by HRHD and DFRP, which results in the elongated time to rupture as compared to those of Gr.II

\subsection{Cr-4W Steel}

\subsubsection{Time to Rupture and Analysis of Creep Curves}

Figure 7 shows the stress vs. time to rupture relationship for $9 \mathrm{Cr}-4 \mathrm{~W}$ steel (Abe et al., 1992). The data were classified into three groups, Grs.I - III, and the regression lines were obtained using the same method as for $9 \mathrm{Cr}-1 \mathrm{~W}$ steel (Section 4.1.1). In the classification, $\sigma-t_{\varepsilon}$ diagrams were referred and the $\sigma-t_{0.5}, t_{2}$, and $t_{5}$ diagrams are shown in Figure A3, as typical examples. Linear relationships between stress and logarithmic time are confirmed in Figure A3 in the same way as shown in Figure 7, which indicates that $t_{\varepsilon}$ can be applied to Equation 1 if we replace $t_{r}$ with $t_{\varepsilon}$. The $t_{r} \mathrm{~s}$ of $G r . I I$ are longer than the extrapolated $t_{r} \mathrm{~s}$ of $G r . I$ for the stresses in Gr.II, i.e., $t_{I I}>t_{I}$, but $t_{I I I}<t_{I I}$ at higher temperatures and lower stresses. At $600{ }^{\circ} \mathrm{C}$, the $t_{r}$ at the highest stress belongs to Gr.I and the $t_{r}$ at the lowest stress belongs to Gr.III and therefore, the $\sigma-t_{r}$ relationship at $600{ }^{\circ} \mathrm{C}$ of Figure 7 or a locus of the regession lines for Grs.I - III appears to change along S-shape with logarithm 
of time, or the degradation in strength seems to occur after several thousands of hours, corresponding to Gr.III. The degradation in strength is clearly confirmed at $650{ }^{\circ} \mathrm{C}$ for longer than 1000 h, i.e., Gr.III. As shown in Figure A3, these trends are similar to the $\sigma-t_{5}$ and $t_{10}$ diagrams, but the $\sigma-t_{2}$ diagram changes in inverse $\mathrm{J}$-shape and the $\sigma-t_{0.25}, t_{0.5}$,and $t_{1}$ diagrams change in Z-shape (the $\sigma-t_{0.25}$ and $t_{1}$ diagrams are omitted).

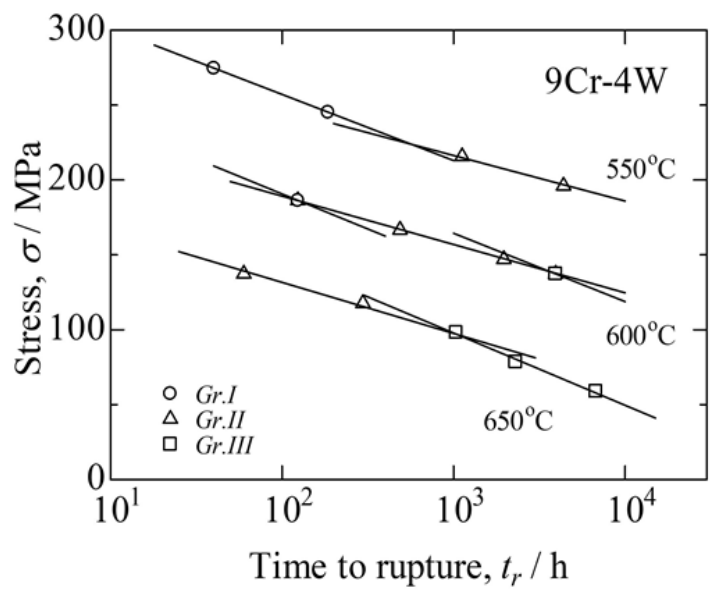

Figure 7. Stress vs. time to rupture relationship for $9 \mathrm{Cr}-4 \mathrm{~W}$ steel

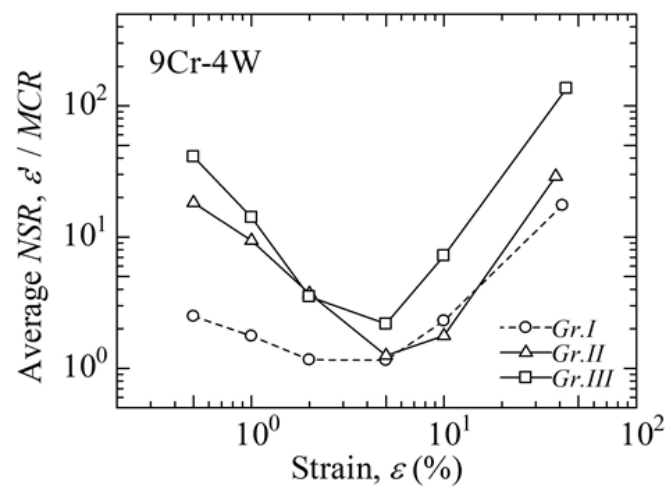

Figure 8 . The average $N S R$ vs. strain relationship for $9 \mathrm{Cr}-4 \mathrm{~W}$ steel

Figure 8 shows the relationship between strain and the average NSR. MCR were observed between $2-5 \%$ strain, but the average NSR for Gr. III at 5\% strain is slightly higher than 2, due to the characteristics of data for Gr.III. Though the average NSRs for Gr.II at an early stage of transient creep are larger than those for Gr.I, the average NSR of Gr.II decreases rapidly and the slope for Gr.II, $\Delta \log ($ average NSR)/ $\log \varepsilon$, is larger than that for Gr.I. That is, rapid strain hardening occurs in transient creep of Gr.II. This trend becomes clearer for Gr.III. The slopes in accelerating creep for all groups are similar with each other. The higher strain hardening in transient creep of Gr.III may be responsible for the shortening of $t_{r}$, i.e., the degradation in strength for Gr.III observed in Figure 7. This degradation tendency is also confirmed for $t_{\varepsilon}$ larger than $\varepsilon>5 \%$ as shown in Figure A3, the details of which are discussed in Sections 4.2.4 - 4.2.6.

Figure 9 shows variations of $Q V C$ as functions of strain for each group. The values of $Q$ at rupture for $G r s . I$ and $I I I$ in Figure 9 are approximately $450 \mathrm{kJmol}^{-1}$ and are a little larger than the activation energy for self-diffusion of the alpha-iron. Moreover, the values of $C$ are $\sim 20$, which are similar values confirmed in many heat-resistant steels (Tamura et al., 2013). On the other hand, $Q \sim 600 \mathrm{kJmol}^{-1}$ and $C \sim 30$ are obtained for Gr.II at rupture, which means that the hard microstructure is maintained until rupture. 

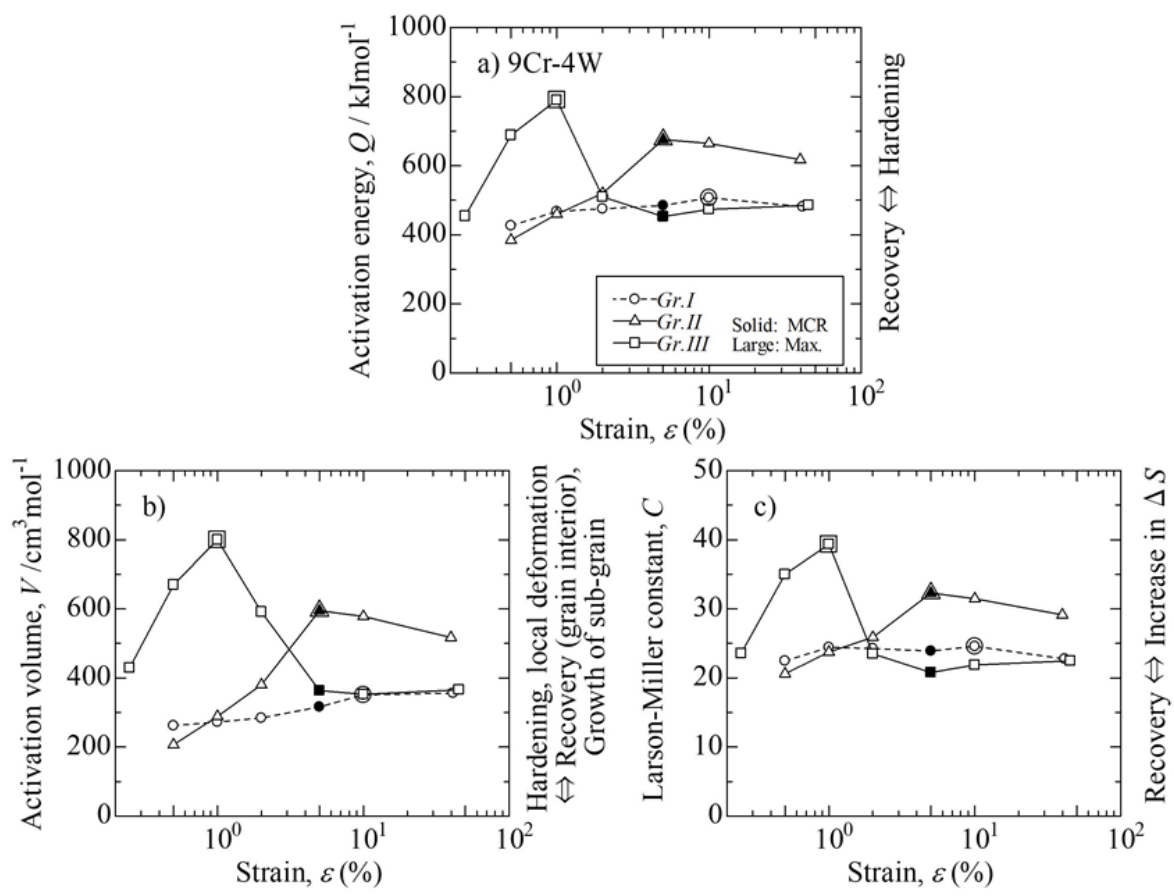

Figure 9. The results of the $Q V C$ analysis are shown as functions of creep strain for each group of 9Cr-4W steel; a) the activation energy, b) the activation volume, and c) the Larson-Miller constant

\subsubsection{Gr.I}

Variations of QVC for Gr.I shown in Figure 9 are not very large, though the data at $0.25 \%$ strain are not obtained with sufficient accuracy. SBSD may occur similar to the early stage of transient creep for Gr.I of $1 \mathrm{~W}$ steel, because $Q$ and $V$ increase slightly up to $10 \%$ strain. The average test conditions are $567{ }^{\circ} \mathrm{C}, 235 \mathrm{MPa}$, and $t_{r}=116 \mathrm{~h}$. Under such conditions, precipitation of the Laves phase hardly occurs (Abe et al., 1991). The recovery inside the sub-grains and the growth of the sub-grains with DFRP for $\mathrm{M}_{23} \mathrm{C}_{6}$ may occur, hence maintaining the lath martensite structure, $M C R$ is observed at $\sim 5 \%$ strain. Accelerating creep at $\varepsilon>10 \%$ is caused by recovery due to decreases in $Q$ and $C$. There is no evidence of obvious precipitation hardening except for $\mathrm{M}_{23} \mathrm{C}_{6}$ throughout the duration of creep, although a few Laves phase particles $(500 \mathrm{~nm}$ in length) were observed on the lath boundaries (Abe, 2003).

\subsubsection{Gr.II}

The average test conditions for Gr. $I I$ are $600{ }^{\circ} \mathrm{C}, 163 \mathrm{MPa}$, and $t_{r}=1546 \mathrm{~h}$. The Laves phase is confirmed under such conditions (Abe, 2005). Remarkable increases in QVC up to 5\% strain indicate strengthening of the sub-boundaries due to precipitation of fine Laves phase and $\mathrm{M}_{23} \mathrm{C}_{6}$ together with SBSD at an early stage of transient creep. Accelerating creep at higher strains of $5 \%$ is caused mainly by the growth of the sub-grains maintaining grain boundary strengthening due to the Laves phase and $\mathrm{M}_{23} \mathrm{C}_{6}$, because high levels of $Q V C$ are retained up to rupture. To investigate the metallurgical reactions during creep in more detail, the $Q_{s}, V_{s}$, and $C_{s}$ analyses were also performed on this group similar to that shown in Table 4. Though the Table is omitted, it was found that all of $Q_{s}, V_{s}$, and $C_{s}$ increase up to 5\% strain and then decrease during accelerating creep and that the determinant term is $Q_{s}$ during the whole creep strain except for the cases in the $0.25-0.5 \%$ strain range, near the $M C R$ point. These results support the metallurgical reactions in transient creep which are inferred based on the $Q V C$ analysis. Moreover, the results show that after the grain boundary strengthening due to the Laves phase and $\mathrm{M}_{23} \mathrm{C}_{6}$ reaches the upper limit, the HRHD process with the DFRP mechanism for $\mathrm{M}_{23} \mathrm{C}_{6}$ begins together with the sub-grain growth in accelerating creep. However, it is found that based on both the QVC and $Q_{S}, V_{s}$, and $C_{s}$ analyses that hardening due to the Laves phase and $\mathrm{M}_{23} \mathrm{C}_{6}$ with fine martensitic structure is almost maintained up to rupture. 


\subsubsection{Gr.III}

The average test conditions for $\mathrm{Gr} . I I I$ are $638^{\circ} \mathrm{C}, 93 \mathrm{MPa}$, and $t_{r}=3507 \mathrm{~h}$. The Laves phase forms under these conditions (Abe, 2005). Rapid increases in QVC up to 1\% strain shown in Figure 9 are caused by the accelerating reactions of fine Laves phase observed in Gr.II together with SBSD at an early stage of transient creep. Therefore, in this strain range, $t_{\varepsilon}$ is longer than those expected from Gr.II (Figure A3). On the other hand, after $1 \%$ strain $Q V C$ turn over decreases significantly and this situation continues up to $5 \%$ strain of the $M C R$ point. Both $Q$ and $C$ decrease between $1-2 \%$ strain to a greater extent than those for $2-5 \%$, whereas $V$ decreases linearly against $\log \varepsilon$ through $1-5 \%$ strain. These decreases in QVC can be essentially explained by the HRHD processes, because the Laves phase precipitates only on the boundaries, but has not been confirmed inside sub-grains in 9Cr-4W steel (Abe, 2005). It is also observed that the values of $Q$ and $C$ at the $M C R$ point are both still smaller than those of Gr.I, where the Laves phase hardly precipitates. Therefore, coarsening of the Laves phase and $\mathrm{M}_{23} \mathrm{C}_{6}$ should take place rapidly during the later stage of transient creep together with the progress of HRHD. The detailed coarsening mechanisms of the Laves phase and $\mathrm{M}_{23} \mathrm{C}_{6}$ will be explained in the later Sections 5.2.5 and 5.2.6. The above-mentioned interpretation on the variations of $Q V C$ in the transient creep for Gr.III must correspond to the trend for the changes in the $\sigma-t_{\varepsilon}$ diagrams shown in Figure A3. Namely, in a $1-2 \%$ strain range, the $\sigma-t_{\varepsilon}$ relationships change in inverse J-shape as shown in Figure A3b) or $t_{I I I}>t_{I I}$, and $Q$ for Gr.III is higher than that for Gr.II as shown in Figure 9. However, in a $2-5 \%$ strain range, the strengthening effect of the Laves phase and $\mathrm{M}_{23} \mathrm{C}_{6}$ is weakened further due to the coarsening of the precipitates by the progress of the HRHD processes, because $Q$ for $G r . I I I$ is smaller than that for Gr.II. Finally, at $5 \%$ strain the $\sigma-t_{\varepsilon}$ relationship changes in S-shape as shown in Figure A3c), or $t_{I I I}<t_{I I}$. In accelerating creep, the values of $Q V C$ are low values similar to those at $M C R$, and therefore strain rate increases recovering the microstructure, accompanying the growth of sub-grains, and coarsening the precipitates together with the HRHD processes.

\subsubsection{Strain Rate Analysis for Gr.III of 4W}

A similar phenomenon to that observed for Gr.II of $4 \mathrm{~W}$ in accelerating creep in which all of $Q V C$ decrease is observed between $1-5 \%$ strain for Gr.III of 4W. However, the phenomenon in Gr.III occurs in transient creep and therefore, the creep mechanism for this phenomenon is different from that of Gr.II. In the case of Gr.III of $4 \mathrm{~W}, V$ decreases in transient creep, i.e., creep behavior between $1-5 \%$ strain for Gr.III of $1 \mathrm{~W}$ steel in the same manner as that for $4 \mathrm{~W}$ steel, but conversely $Q$ and $C$ decrease for $4 \mathrm{~W}$ steel, whereas in $9 \mathrm{Cr}-1 \mathrm{~W}$ steel both $Q$ and $C$ increase. Except for the degrees of the changes in $Q V C$, the most similar case to that of Gr.III of $9 \mathrm{Cr}-4 \mathrm{~W}$ steel, is the creep behavior of $G r . I$ of $9 \mathrm{Cr}-1 \mathrm{~W}$ steel in the $1-2 \%$ strain range. To clarify the creep behavior for Gr.III of $4 \mathrm{~W}$ steel between $1-5 \%$ strain, the $Q_{s}, V_{s}$, and $C_{s}$ analyses were made using the same method shown in Figure 5 and Table 4. The results are shown in Figure 10 and Table 7. The changes in the obtained $Q_{s}, V_{s}$, and $C_{s}$ shown in Figure 10 are similar to those of $Q V C$ in Figure 9. However, that all of $Q_{s}, V_{s}$, and $C_{s}$ increase clearly in Figure 10 between $5-10 \%$ strain, i.e., in accelerating creep excepting for the data at rupture, is differ from those for QVC in Figure 9.

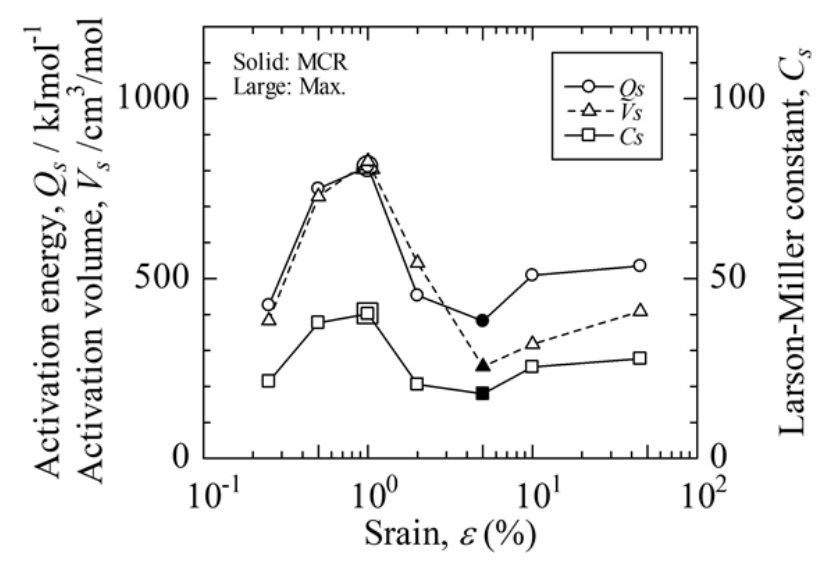

Figure 10. $Q_{s}, V_{s}$, and $C_{s}$ for $G r . I I I$ of $9 \mathrm{Cr}-4 \mathrm{~W}$ steel are shown as functions of creep strain 
It is found from Table 7 that the determinant term for $\dot{\varepsilon}$ up to $1 \%$ strain is the increase in $\left[Q_{s}\right]$, which is caused by the grain boundary strengthening due to the dislocations that are swept out, $\mathrm{M}_{23} \mathrm{C}_{6}$, and Laves phase, and the determinant term for $\dot{\varepsilon}$ in accelerating creep is the increase in $C_{s}$. Between $1-5 \%$ strain, both $C_{s}$ and $\left[V_{s}\right]$ are the determinant terms contributing to the decreased $\dot{\varepsilon}$ in transient creep. If we are only observing the changes in large values i.e., $\left[Q_{s}\right]$ and $C_{s}$, it can be easily misconstrued that accelerating creep begins from $1 \%$ strain, because $\left[Q_{s}\right]$ decreases significantly. Between $1-5 \%$ strain, the decrease in $\dot{\varepsilon}$ cannot be explained only by the decrease in $C_{s}$, and therefore, a decrease in $\left[V_{s}\right]$ or HRHD is absolutely necessary to maintain transient creep.

Table 7. Changes in $Q_{s}, V_{s}, C_{s}$, and $\log (\dot{\varepsilon})$ between two adjacent strains for $G r . I I I$ of $9 \mathrm{Cr}-4 \mathrm{~W}$ steel. $M C R$ is near $5 \%$ strain

\begin{tabular}{ccccccc}
\hline Strain range & {$[0.25-0.5 \%]$} & {$[0.5-1 \%]$} & {$[1-2 \%]$} & {$[2-5 \%]$} & {$[5-10 \%]$} & {$\left[10 \%-t_{r}\right]$} \\
\hline$-\Delta\left[Q_{S}\right]$ & $\mathbf{- 1 8 . 6 0}$ & $\mathbf{- 3 . 5 2}$ & 20.55 & 4.08 & -7.29 & -1.49 \\
$\Delta\left[V_{S}\right]$ & 1.85 & 0.52 & $-\mathbf{1 . 5 1}$ & $\mathbf{- 1 . 5 4}$ & $\mathbf{0 . 3 3}$ & $\mathbf{0 . 4 9}$ \\
$\Delta C_{S}$ & 16.32 & 2.47 & $\mathbf{- 1 9 . 6 1}$ & $\mathbf{- 2 . 6 2}$ & $\mathbf{7 . 4 7}$ & $\mathbf{2 . 3 0}$ \\
$\log (\dot{\varepsilon}, \% / \mathrm{h})$ & -0.43 & -0.52 & -0.58 & -0.08 & 0.51 & 1.29 \\
\hline
\end{tabular}

In other words, the Laves phase particles grow rapidly in the HRHD zone formed, which causes the large decreases in $\left[Q_{s}\right]$ and $C_{s}$ and consequently transient creep is maintained when $C_{s}+\left[V_{s}\right]<\left[Q_{s}\right]$. This process propagates repeatedly throughout the entire specimen and thus $t_{\varepsilon}$ for $\varepsilon<2 \%$ for Gr.III is longer than that estimated from Gr.II as shown in Figure A3. However, an increase in $C_{s}$ is the determinant term in accelerating creep, where $t_{\varepsilon}$ for Gr.III is shorter than that estimated from Gr.II as shown in Figure 7 and Figure A3c). During this strain range either recovery inside the sub-grains or the growth of sub-grains or both occur, because $V_{s}$ increases. However, HRHD is also expected to occur, because the value of $V_{s}$ is rather small. Therefore, Table 7 shows that accelerating creep is caused by the fact that the increase in the entropy term associated with the local movement of dislocations is larger than the increase in $\left[Q_{s}\right]$. Although the rather large increase in $\left[Q_{s}\right]$ during accelerating creep appears to be unusual, this phenomenon is not unique because similar phenomena, often accompanying HRHD, are seen in the cases not only for Grs.I and II of $1 \mathrm{~W}$ and Gr.I of 4W, but also for Grs.IIIa of T92/MJT and T91/MGC, respectively, mentioned later.

\subsubsection{Degradation in Creep Strength for Gr.III}

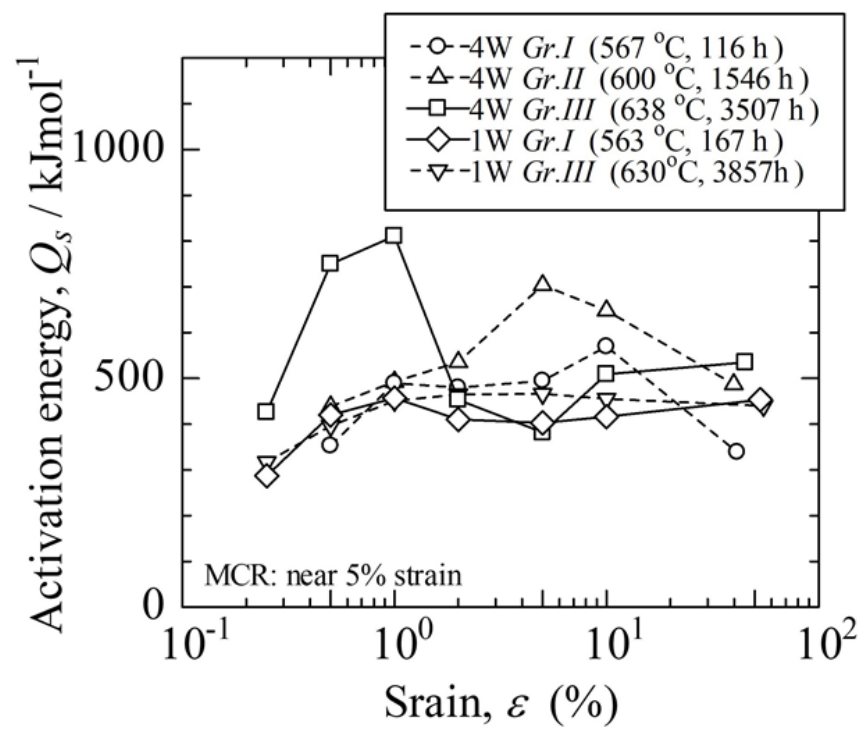

Figure 11. Activation energy, $Q_{S}$, as functions of strain for $9 \mathrm{Cr}-1 \mathrm{~W}$ and $9 \mathrm{Cr}-4 \mathrm{~W}$ steels. The average temperature and time to rupture are shown in the figure for each data group 
Creep strength for Gr.III of 4W steel decreases compared to those expected from Gr.II when $\varepsilon>5 \%$ (Figures 7 and A3c)). Figure 11 shows the comparison among the $Q_{s}$ s for $1 \mathrm{~W}$ and $4 \mathrm{~W}$ as functions of strain. Among the $Q_{s}-\varepsilon$ relationships, those for Gr.I of $1 \mathrm{~W}$ and Gr.III of $4 \mathrm{~W}$ are drawn using real lines, because they are phenomenologically very similar to each other. They show a peak at $1 \%$ strain, a minimum near the $M C R$ point, and an increase in accelerating creep. Moreover, the $V \mathrm{~s}$ for both data groups also decrease between $1 \%$ strain to each $M C R$ point, which clearly indicates the occurrence of HRHD (Figures 4 and 9). However, it is understood that the occurrence of HRHD may not be a direct cause for the degradation of strength, because in the $\sigma-t_{2}$ diagram for $4 \mathrm{~W}$ shown in Figure A3b) the degradation in strength does not occur as yet, but HRHD definitely occurs at $\varepsilon=2 \%$ for Gr.III of $4 \mathrm{~W}$ (Figures 9 and 10), because $V$ decreases distinctly in this strain range.

Table 8. The differences in QVC for Grs.II and $I I I$ at an indicated strain of $9 \mathrm{Cr}-4 \mathrm{~W}$ steel and the calculated $t_{I I}$ and $t_{I I I}$ at the average temperature and stress for Gr.III. Key factors determining $\log \left(t_{I I I} / t_{I I}\right)$ are shown in the bottom. Determining term(s) for $\log \left(t_{I I I} / t_{I I}\right)$ is (are) shown in bold-italics

\begin{tabular}{ccccccc}
\hline Strain $(\%)$ & 0.5 & 1 & 2 & 5 & 10 & 39.8 \\
\hline$\Delta[Q]$ & $\mathbf{1 7 . 4 4}$ & $\mathbf{1 9 . 0 0}$ & -0.57 & $-\mathbf{1 2 . 7 8}$ & $-\mathbf{1 0 . 9 6}$ & -7.57 \\
$-\Delta[V]$ & -2.48 & -2.74 & -1.13 & 1.23 & 1.20 & 0.80 \\
$-\Delta C$ & -14.39 & -15.59 & 2.39 & 11.51 & 9.58 & 6.66 \\
$\log \left(t_{I I I} / t_{I I}\right)$ & 0.58 & 0.67 & 0.69 & -0.04 & -0.18 & -0.11 \\
& SBSD, $\mathrm{M}_{23} \mathrm{C}_{6}$ and Laves phase & Recovery due to $\Delta C<0$ & Recovery due to coarsening of Laves, \\
& hardening $(\varepsilon<1 \%)$ & $(\varepsilon \sim 2 \%)$ & \multicolumn{3}{c}{$\mathrm{M}_{23} \mathrm{C}_{6},(\varepsilon \geq 5 \%)$} \\
\hline
\end{tabular}

Moreover, there is an issue because at $\varepsilon=5 \%$, near the $M C R$ point of $4 \mathrm{~W}$ the minimum $Q_{s}\left(382.0 \mathrm{kJmol}^{-1}\right)$ of Gr.III of $4 \mathrm{~W}$ is smaller than that of $G r . I$ of $1 \mathrm{~W}\left(402.7 \mathrm{kJmol}^{-1}\right)$. The fact that $Q_{s}(4 \mathrm{~W})<Q_{s}(1 \mathrm{~W})$ at $\varepsilon=5 \%$ indicates that the Laves phase particles in $4 \mathrm{~W}$ grow extremely large through the HDHD processes and the strengthening effect is completely lost, though the Laves phase surely contributes to strengthening up to $1 \%$ strain. If we correlate this fact with the observations that massive Laves phase particles are formed nearby or adjacent to $\mathrm{M}_{23} \mathrm{C}_{6}$ particles in Grade 92 (Dimmler et al.,2003; Dudko et al., 2013; Nie et al., 2014; Xu et al., 2015; Maddi et al., 2016), the following hypothesis is possible: The vicious cycle of the coarsening of both $\mathrm{M}_{23} \mathrm{C}_{6}$ and the Laves phase begins with HRHD as momentum, the Laves phase decomposes some of the $\mathrm{M}_{23} \mathrm{C}_{6}$ particles and absorbs the decomposed soluble $\mathrm{W}$ atoms and therefore, not only does the Laves phase grow further but also $\mathrm{M}_{23} \mathrm{C}_{6}$ with a lower $\mathrm{W}$ content grows rapidly, which results in loss of the strengthening factors of both $\mathrm{M}_{23} \mathrm{C}_{6}$ and the Laves phase. When this is considered, the main reason for the degradation observed in Gr.III of $9 \mathrm{Cr}-4 \mathrm{~W}$ steel for $\varepsilon>5 \%$ is believed to be the extreme coarsening of the Laves phase and $\mathrm{M}_{23} \mathrm{C}_{6}$ caused by the formation of the HRHD as an underlying trigger. The above hypothesis suggests that material design based on the grain boundary strengthening using precipitates such as the Laves phase is effective according to the test conditions, but at high temperatures and for long-term tests HRHD triggers extreme coarsening of precipitates during accelerating creep, and consequently the degradation in strength. To prevent degradation of this type, it is necessary to moderate the excess Laves phase hardening during transient creep or before testing.

Rupture strength of Gr.III shown in Figure 7 decreases clearly as compared with the expected values from Gr.II. However, it is found from Figures 7 and A3c) that the strength degradation occurs only when $\varepsilon>5 \%$ and on the contrary rupture strength of Gr.III is higher than that expected from Gr.II for time to smaller strains. To investigate this phenomenon metallurgically, a similar QVC analysis as shown in Table 6 for Gr.III of 9Cr-1W steel was made under the average test conditions of Gr.III. The results are shown in Table 8. It is found from Table 8 that at $\varepsilon \geq 5 \%$ degradation in strength is determined by decrease in $[Q]$ which is caused by the growth of sub-grains and coarsening of the Laves phase and $\mathrm{M}_{23} \mathrm{C}_{6}$ and that for $\varepsilon \leq 1 \%$ an increase in strength is determined by an increase in $[Q]$ which is caused SBSD and grain boundary strengthening due to $\mathrm{M}_{23} \mathrm{C}_{6}$ and the Laves phase. It is also noticed that for $\varepsilon \geq 2 \%$ the change in $\Delta C$ of the entropy term due to HRHD begins to decrease and for $\varepsilon=2 \%$ of transient creep, $t_{2}$ in Gr.III becomes larger than that in Gr.II, because the decrease in $\Delta C$ becomes larger than the decrease in $\Delta[Q]$. As shown in Figures 2 and 7, though the remarkable degradation in rupture strength is observed in Gr.III of 4W steel, on the contrary the prolonged time to rupture is observed in Gr.III of 1W steel. In both cases, recovery of martensite structure caused by HRHD takes place, however the estimated rupture strength of $4 \mathrm{~W}$ at $600{ }^{\circ} \mathrm{C}$ for $10000 \mathrm{~h}, 50 \mathrm{MPa}$, is clearly higher than the estimated strength of $31 \mathrm{MPa}$ for $1 \mathrm{~W}$ steel under the same conditions. Namely, it is also reminded that though the microstructure of $4 \mathrm{~W}$ is decomposed 
destructively at rupture due to HRHD, the rupture strength at high temperatures is still stronger than that of $1 \mathrm{~W}$ steel without Laves phase. The values of $Q$ and $C$ for $1 \mathrm{~W}$ and $4 \mathrm{~W}$ steel are approximately the same with each other as shown in Figures 4 and 9, but the value $V$ for $4 \mathrm{~W}, 366 \mathrm{~cm}^{3} \mathrm{~mol}^{-1}$, is about half of that for $1 \mathrm{~W}$ steel $766 \mathrm{~cm}^{3} \mathrm{~mol}^{-1}$. This indicates that the movable area for the dislocations for $4 \mathrm{~W}$ is narrower than that for $1 \mathrm{~W}$ steel, which corresponds to the difference in the sub-grain size, because there are few obstacles inside sub-grains in both cases of Gr.III. By the way, the widths of lath martensite for $1 \mathrm{~W}$ and $4 \mathrm{~W}$ aged at $600{ }^{\circ} \mathrm{C}$ for $3000 \mathrm{~h}$ are $0.75 \mu \mathrm{m}$ and $0.53 \mu \mathrm{m}$, respectively (Abe et al., 1991), and the sub-grain size of $4 \mathrm{~W}$ at rupture may be judged to be smaller than that of $1 \mathrm{~W}$ steel. The reason for this is considered to be caused by the fact that $\mathrm{M}_{23} \mathrm{C}_{6}$ in $4 \mathrm{~W}$ is finer than that in $1 \mathrm{~W}$ because of the higher content of $\mathrm{W}$ in $4 \mathrm{~W}$ steel (Yoshizawa et al., 2005; Abe, 2006a; Danielsen, 2007). This also suggests that stabilizing the sub-grain size utilizing particles like $\mathrm{M}_{23} \mathrm{C}_{6}$ is very important to prevent the degradation in long-term rupture strength of martensitic steel.

\subsection{P92/MJP}

\subsubsection{Time to Rupture and Analysis of Creep Curves}

Figure 12 shows the $\sigma-t_{r}$ relationship for P92/MJP (NIMS, 2018). Creep tests were conducted at a wide range of test conditions and the data were classified into 6 groups. The $\sigma-t_{r}$ relationship between $600-$ $650{ }^{\circ} \mathrm{C}$ and below about $10000 \mathrm{~h}$ changes along S-shape like those of $9 \mathrm{Cr}-4 \mathrm{~W}$ (Figure 7) and so, the data groups were named Grs.I - III. The data groups at higher temperatures and for longer terms were named Gr.IIa and Gr.IIIa, respectively. The data group at higher temperatures and for longer terms was named Gr.IV as shown in Figure 12. The regression analyses for each data group were made using Equation 3 and the regression lines are shown in the figure using the $Q V C$ s obtained. In classifying the data, the $\sigma-t_{\varepsilon}(\varepsilon=0.5-5 \%)$ and $\sigma-t_{M C R}$ diagrams were referred. The $\sigma-t_{1}$ diagram is shown in Figure A4 as a typical example. The rupture data of many newly developed martensitic steels with high strength are concentrated between $600-650{ }^{\circ} \mathrm{C}$ and for some 100s to $10000 \mathrm{~h}$, which corresponds to Gr.II in Figure 12. Hardening in Gr.II of MJP is confirmed, because the $t_{r}$ of Gr.II is longer than that estimated from the data in Gr.I. This hardening is considered to be caused by the precipitation of the Laves phase from the previous work (Hasegawa et al., 2003; 2004; Kipelova et al., 2012) and Figure 7. Degradation already begins from Gr.III as compared with the data of Gr.II. The unexpected drop in strength of Grade 92 highlighted by Sawada et al. (2007) corresponds to Gr.IIIa in Figure 12, where the slopes of the regression lines for Gr.IIIa are steeper than those of Gr.III. On the other hand, the slope of each line for Gr.IV at higher temperatures is slower than that for Gr.IIIa. Similarly, the rupture behavior of high strength steel around $100000 \mathrm{~h}$ is complex, though $t_{r}$ changes in S-shape over the long term. The rupture data for much longer terms are required to facilitate a detailed investigation of the rupture behavior of high strength steel.

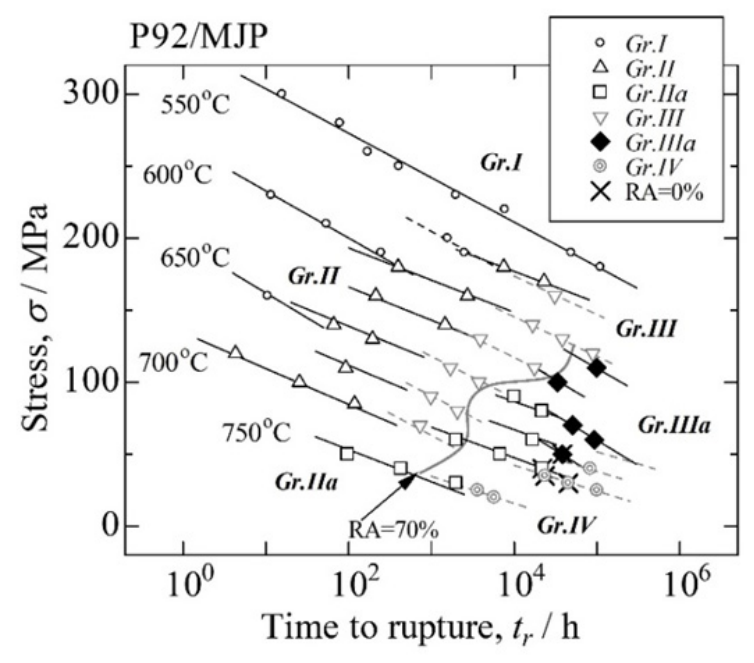

Figure 12. The stress vs. time to rupture relationship for P92/MJP and regression lines for each data group. Creep tests were conducted at $25^{\circ} \mathrm{C}$ intervals, but test temperatures are only shown in every $50{ }^{\circ} \mathrm{C}$ (this documentation is the same for other similar diagrams, and hereinafter omitted). The $R A$ s for the bottom right of a spool curve are below $70 \%$ 
The curve in Figure12 is a spool line based on the interpolated data at $R A=70 \%$ using an $R A$ vs $t_{r}$ diagram. The $R A$ s for the bottom right of the spool line are below $70 \%$ and the crosses in the figure denote $R A=0 \%$, though the $E L$ for each is around $10 \%$. Moreover, $R A$ tends to recover for a longer time duration. The decrease in ductility is mainly caused by the pre-existing BN particles (Gu et al., 2014; Parker \& Siefert, 2018; Abe et al., 2018). Breakdown in rupture strength of Gr.IIIa definitely occurs in the degradation region of $R A$, however the primary cause for the breakdown in strength should be investigated separately from $\mathrm{BN}$, because the center of Gr.IIIa is positioned at a higher stress in Figure 12 than the center of the $R A=0$ zone.

As shown in Figure A4 the linear relationships between $\sigma$ and $\log t_{\varepsilon}$ for $\varepsilon=0.5-5 \%$ were already confirmed for each group. Therefore, it is reasonable that $t_{\varepsilon}$ is applied to Equation 1 when we replace $t_{r}$ with $t_{\varepsilon}$. However, since Equation 1 is introduced using the Mankman-Grant relation, Equation 6, it is desirable to confirm the relation between $M C R$ and $t_{\varepsilon}$. Figure A5 shows the relationships between $M C R$ vs. $t_{r}$ and $t_{\varepsilon}$ for P92/MJP. Though the scattering for $t_{0.5}$ is rather large, the validity of applying Equation 1 to $t_{\varepsilon}$ is reconfirmed from Figures A4 and A5.

Figure 13 shows the $\varepsilon$-average NSR relationship for each data group of P92/MJP. The average NSRs for Grs.IIa, IIIa, and $I V$ which range at higher temperatures and for longer terms in the $\sigma-t_{\varepsilon}$ diagrams are rather small in transient creep and rapid strain hardening occurs in these groups followed by each $M C R$ at a small strain of $1 \%$. On the other hand, in accelerating creep the average NSRs for Gr.III and Gr.IIIa including Gr.IV which range for longer terms in Figure 12 increase rapidly compared to other groups. Particularly in Gr.IIIa, where the break down in rupture strength has been questioned, it is considered that the rapid strain hardening in transient creep triggers the subsequent increase in creep rate in accelerating creep which leads to the break down in rupture strength.

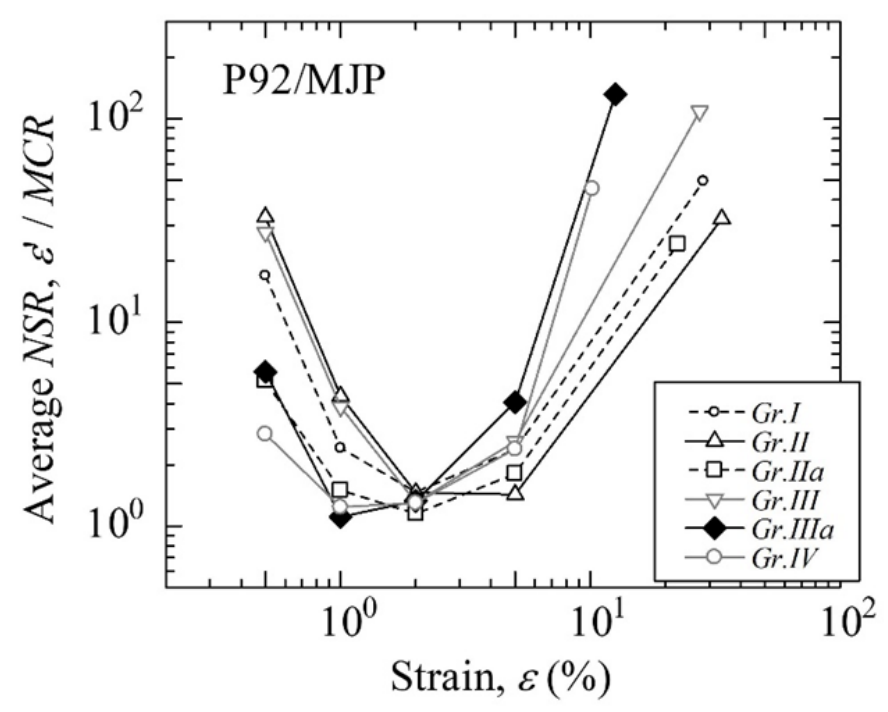

Figure 13. The average NSR vs. strain relationship for P92/MJP

Figure 14 shows the variations of QVC as functions of strain for each group. The Z-phase is formed in Grs.III, IIIa, and $I V$ and the strains corresponding to time to initiate the Z-phase formation (Sawada et al., 2007) are indicated by arrows (this documentation is the same for other similar diagrams, and hereafter omitted). The values of $Q$ at rupture for Grs. IIIa and $I V$ where the break down in rupture strength is observed in Figure 12, are approximately $500 \mathrm{kJmol}^{-1}$ and are a little larger than the activation energy for self-diffusion of the alpha-iron. Moreover, the values of $C$ are $\sim 23$, which are similar values confirmed in many heat-resistant steels (Tamura et al., 2013). On the other hand, $Q>600 \mathrm{kJmol}^{-1}$ and $C>30$ are obtained at rupture for other groups, which means that the hard microstructure is maintained until rupture.

\subsubsection{Gr.I}

The average test conditions are $570{ }^{\circ} \mathrm{C}, 217 \mathrm{MPa}, t_{0.5}=319 \mathrm{~h}$, and $t_{r}=12377 \mathrm{~h}$. The Laves phase is not formed in the average test conditions for Gr.I (Haetterstrand \& Andren, 2001; Zielinski et al., 2016). The 
changes in $Q V C$ shown in Figure 14 are not so large, $Q \sim 800 \mathrm{kJmol}^{-1}, V \sim 500 \mathrm{~cm}^{3} \mathrm{~mol}^{-1}$, and $C \sim 40$ and therefore, the microstructure recovers with a slow pace during creep maintaining the martensite lath structure with $\mathrm{M}_{23} \mathrm{C}_{6}$ and fine MX particles. However, SBSD may take place within $\varepsilon<1 \%$, because both $Q$ and $V$ increase slightly in transient creep.

\subsubsection{Gr.II}

All of QVC increase distinctly up to $2 \%$ strain which shows the occurrence of recovery inside the sub-grains and SBSD. This phenomenon is similar to the case of the initial stage of Gr.II of $9 \mathrm{Cr}-4 \mathrm{~W}$ steel and thus sub-grain boundaries are strengthened by the pre-existing $\mathrm{M}_{23} \mathrm{C}_{6}$ and newly precipitated Laves phase together with SBSD. The average test conditions are $640{ }^{\circ} \mathrm{C}, 140 \mathrm{MPa}, t_{1}=139 \mathrm{~h}, t_{2}=1015 \mathrm{~h}$ and $t_{r}=2974 \mathrm{~h}$. Around $t_{2}$,
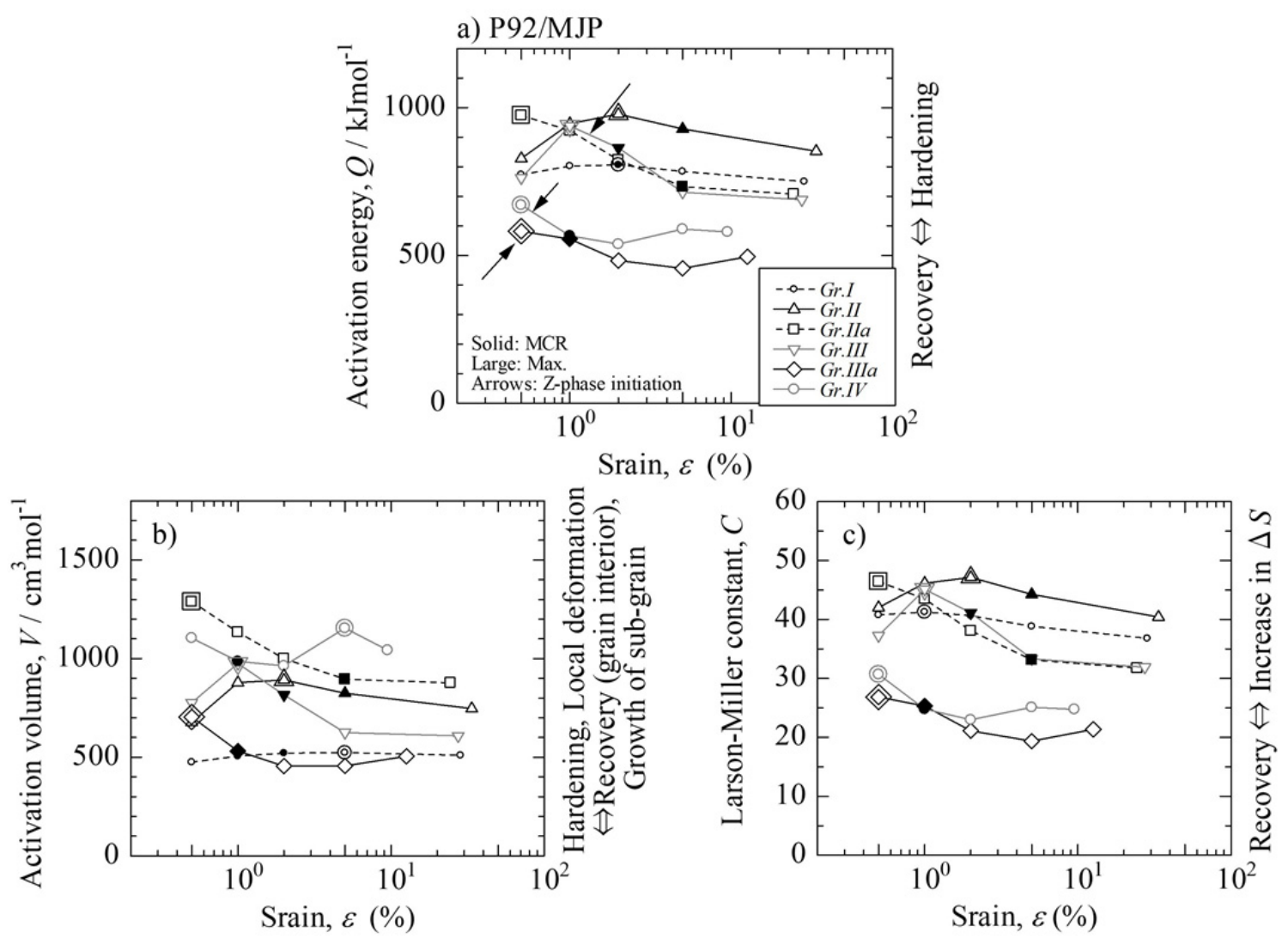

Srain, $\varepsilon(\%)$

Figure 14. The results of the QVC analysis are shown as functions of creep strain for each group of P92/MJP steel; a) the activation energy, b) the activation volume, and c) the Larson-Miller constant. Allows indicate strains corresponding to the beginning time for the formation of Z-phase (omitted this explanation, hereinafter)

precipitation of the Laves phase was experimentally confirmed by Haetterstrand and Andren (2001), Hasegawa et al. (2003; 2004), and Zielinski et al. (2016). The Z-phase is not formed in the average rupture conditions (at $640{ }^{\circ} \mathrm{C}$ for $2974 \mathrm{~h}$ ) of $\mathrm{Gr} . I I$ for P92/MJP referring to the data for Grade T92 (Sawada et al., 2007). After $\varepsilon>2 \%$, HRHD begins at the weak portions of the sub-boundaries strengthened by SBSD followed by recovery and coarsening of the precipitates, because both $Q$ and $V$ begin to decrease, the Laves phase does not form inside the sub-grains (Hasegawa et al., 2003 and 2004), and new other precipitation does not occur. Coarsening of the Laves phase also occurs during creep, but the value of $Q$ at rupture is rather high, $Q \sim 887 \mathrm{kJmol}^{-1}$, compared to that of Gr.I and so the strengthening effect due to the Laves phase was considered to be continued up to rupture.

\subsubsection{Gr.IIa}

The values of $Q V C$ at $\varepsilon=0.5 \%$ become the maximums with each among the data analyzed, then $Q V C$ 
decrease up to $\varepsilon=5 \%$ and keep approximately constant. The average test conditions are $703{ }^{\circ} \mathrm{C}, 56 \mathrm{MPa}$, $t_{0.5}=458 \mathrm{~h}$ and $t_{r}=9083 \mathrm{~h}$. The value of $Q \sim 1000 \mathrm{kJmol}^{-1}$ at $\varepsilon=0.5 \%$ is far higher than $Q \sim 450 \mathrm{kJmol}^{-1}$ due to SBSD in $1 \mathrm{~W}$ steel and further higher than $Q \sim 800 \mathrm{kJmol}^{-1}$ due to SBSD, the Laves phase, and $\mathrm{M}_{23} \mathrm{C}_{6}$ in $4 \mathrm{~W}$ steel. The high maximum value of $Q$ in P92/MJP may be caused by higher dislocation density, finer lath martensite structure than $1 \mathrm{~W}$ and $4 \mathrm{~W}$ steel, and also by MX particles. Nie et al. (2014) reported that rather fine Laves phase precipitates of about $200 \mathrm{~nm}$ along sub-boundaries in a ruptured specimen of Grade T92 at $700{ }^{\circ} \mathrm{C}$ for $t_{r}=523 \mathrm{~h}$ the conditions of which are nearly equal to those of Gr.IIa. Nie et al. also reported that the Laves phase grew up to $350 \mathrm{~nm}$ at $700{ }^{\circ} \mathrm{C}$ for $t_{r}=2231 \mathrm{~h}$. This time corresponds to $\varepsilon=1-2 \%$ in an $\varepsilon-Q$ diagram shown in Figure 14a). The value of $V \sim 1300 \mathrm{~cm}^{3} \mathrm{~mol}^{-1}$ at $\varepsilon=0.5 \%$ is considerably large, which indicates the occurrence of sub-grain growth and recovery inside the sub-grains. HRHD should also happen from $\varepsilon=0.5$ to $5 \%$, because both $Q$ and $V$ decrease in this strain range for the same reasons as mentioned for Gr.III of $4 \mathrm{~W}$ steel (Section 4.2.6). In these circumstances $Q$ decreases due to the growth of the Laves phase and $\mathrm{M}_{23} \mathrm{C}_{6}$, but the Z-phase is not yet formed in Gr.IIa (Sawada et al., 2007) and the DFRP mechanism may operate on $\mathrm{M}_{23} \mathrm{C}_{6}$ and therefore, the decrease in $Q$ becomes limited. When the sub-grains grow during accelerating creep, the observed values of $V$ increase which balances the decrease in $V$ due to HRHD.

\subsubsection{Gr.III}

The QVC behavior of Gr.III lies between those of Grs.II and IIa. Up to $\varepsilon=1 \%$ the behavior of Gr.III is similar to that of Gr.II and after $\varepsilon=1 \%$ the behavior is similar to that of Gr.IIa. The average test conditions are $634{ }^{\circ} \mathrm{C}, 113 \mathrm{MPa}, t_{0.5}=123 \mathrm{~h}, t_{1}=1857 \mathrm{~h}, t_{2}=9453 \mathrm{~h}, t_{5}=17565 \mathrm{~h}$, and $t_{r}=18618 \mathrm{~h}$. The start time of the formation of the Z-phase at an average temperature of Gr.III is $\sim 3700 \mathrm{~h}$ according to the TTP diagram for Grade T92 (Sawada et al., 2007) obtained using ruptured specimens of T92. This corresponds to time immediately after $\varepsilon=1 \%$ and long before an $M C R$ point, neglecting the effect caused by the difference between T92 and P92. HRHD should occur at a strain of $\varepsilon>1 \%$, because both $Q$ and $V$ decrease simultaneously. To detect the metallurgical factors by which one determine the creep rate at each strain, the $Q_{s}$, $V_{S}$, and $C_{S}$ analyses were made on Gr.III and the results are shown in Table 9. It is found from Table 9 that the determinant term for $\dot{\varepsilon}$ up to $\varepsilon=1 \%$ is an increase in $\left[Q_{s}\right]$, which is caused by the grain boundary strengthening by the dislocations that are swept out, $\mathrm{M}_{23} \mathrm{C}_{6}$, and the Laves phase, and between $\varepsilon=1 \%$ and an $M C R$ point decreases in $\left[V_{s}\right]+C_{s}$ are the determinant terms. Figure 13 showed that the MCR point for Gr.III is near $\varepsilon=2 \%$, but Table 9 shows that the change in the strain rate between $\varepsilon=2-5 \%$ is negative, i.e., this strain range lies within transient creep. $\left[Q_{s}\right]$ decreases in this strain range and part of the decrease in $\left[Q_{s}\right]$ is caused by the formation of the Z-phase consuming the strengthening particles of MX. Therefore, the decrease in $\left[Q_{s}\right]$ should not be a determinant factor for the decrease in $\dot{\varepsilon}$. In this strain range HRHD should occur because the $\left[V_{s}\right]$ decreases with an increase in strain. In the later stage of transient creep $\left[V_{s}\right]+C_{s}$ is the determinant term and transient creep continues by the decrease in $C_{s}$ caused by the extreme recovery in the HRHD zone. In Figure 14, $V$ remains approximately constant from $\varepsilon=5 \%$ to rupture. In this strain range, the decrease in $V$ due to HRHD is balancing the increase in $V$ due to the growth of sub-grains as discussed in the case of Gr.IIa (Section 4.3.4). The value of $Q$ is also approximately constant in accelerating creep and the level is just the same as that for Gr.II and therefore, in spite of the negative effect of the Z-phase formation both $\mathrm{M}_{23} \mathrm{C}_{6}$ and $\mathrm{MX}$ may still be dispersed finely. As shown in Figure 12, the time to rupture in Gr.III are shorter than those estimated from Gr.II. Hence, factorial analyses were performed for $t_{r}$ and $t_{\varepsilon}$ of Grs.II and III under the average test conditions of Gr.III adopting the same method as in Table 6. The results are shown in Table 10. The detrimental factors for $\log \left(t_{I I} / t_{I I}\right)$ are shown in bold-italics. In $\varepsilon>1 \%$, a decrease in the activation energy, $\Delta[Q]$, is the detrimental term for the degradation of $t_{r}$ and $t_{\varepsilon}$ for Gr.III, which is mainly caused by the formation of the Z-phase consuming the strengthening particles of MX.

Table 9. Changes in $Q_{s}, V_{s}, C_{s}$, and $\log (\dot{\varepsilon})$ between two adjacent strains for Gr.III of P92/MJP steel

\begin{tabular}{ccccc}
\hline Strain range & {$[0.5-1 \%]$} & {$[1-2 \%]$} & {$[2-5 \%]$} & {$\left[5 \%-t_{r}\right]$} \\
\hline$-\Delta\left[Q_{S}\right]$ & $-\mathbf{1 1 . 7 1}$ & 6.11 & 15.40 & $\mathbf{1 . 2 7}$ \\
$\Delta\left[V_{S}\right]$ & 1.51 & $\mathbf{- 2 . 9 7}$ & $\mathbf{- 3 . 0 4}$ & -0.72 \\
$\Delta C_{S}$ & 9.39 & $\mathbf{- 5 . 1 7}$ & $\mathbf{- 1 3 . 0 6}$ & -0.04 \\
$\log (\dot{\varepsilon}, \% / \mathrm{h})$ & -0.81 & -2.03 & -0.71 & 0.52 \\
\hline
\end{tabular}




\subsubsection{Gr.IIIIa}

Break down in the rupture strength in Gr.IIIa is the most remarkable and the values of QVC are the smallest through the whole strain range among all the groups. The smallest values are comparable to those for Grs.III of 1W and 4W steels without MX particles and therefore, the specimens in Gr.IIIa of P92/MJP are considered to be ruptured in a sufficiently recovered state. The average test conditions are $634{ }^{\circ} \mathrm{C}, 78 \mathrm{MPa}, t_{0.5}=4968 \mathrm{~h}$, $t_{1}=27404 \mathrm{~h}, t_{2}=48543 \mathrm{~h}, t_{5}=66550 \mathrm{~h}$, and $t_{r}=62811 \mathrm{~h}$. Here, $t_{5}>t_{r}$ because $t_{r}$ at $625{ }^{\circ} \mathrm{C}$ under $80 \mathrm{MPa}$ is not yet obtained. The sign of the degradation in strength is already confirmed for $t_{0.5}$, but the degradation is clearly confirmed after $t_{1}$, as shown in Figure A4. The start time of the Z-phase formation at an average temperature of Gr.IIIa is $\sim 3700 \mathrm{~h}$ according to the TTP diagram for Grade T92 (Sawada et al., 2007). This time corresponds to time before $t_{0.5}=4968 \mathrm{~h}$. The TTP diagram was obtained using ruptured specimens of Grade T92 and creep strain tends to accelerate the formation of the Z-phase (Sawada et al., 2006; Sawada, Kushima, Tabuchi, \& Kimura 2014b) and so, the formation of the Z-phase may be insufficient for $t_{0.5}$ and moreover, strictly speaking the difference in the formability of the Z-phase between Grades T92 and P92 should also be considered. Haetterstrand and Andren (2001) confirmed the Z-phase in Grade P92 by employing TEM in aged specimens at $600{ }^{\circ} \mathrm{C}$ and $650{ }^{\circ} \mathrm{C}$ for $26000 \mathrm{~h}$ and Yoshizawa et al. (2009) confirmed the formation of the Z-phase and the disappearance of a part of MX particles in an aged specimen at $650{ }^{\circ} \mathrm{C}$ of a laboratory melt type-92 steel plate up to $21000 \mathrm{~h}$. Therefore, the Z-phase is considered to be already formed consuming a part of the MX particles in P92/MJP for $t_{1}=27404 \mathrm{~h}$, near the $M C R$ point, where the degradation in strength was clearly confirmed. All of the QVC decrease between $\varepsilon=0.5-2 \%$ and so, the HRHD is in progress accompanying the formation and growth of precipitates including the Z-phase near the recovered zone. To detect the metallurgical factors which determine the creep rate at each strain, the $Q_{s}, V_{s}$, and $C_{s}$ analyses were made on Gr.III $a$ and the results are shown in Table 11. In a transient creep, $\varepsilon \leq 1 \%$, the decrease in $\Delta\left[V_{s}\right]+\Delta C_{s}$ is the determinant term, even though $\Delta\left[Q_{s}\right]$ decreases due to the coarsening of precipitates and formation of the Z-phase. Here, the decrease in $\Delta\left[V_{s}\right]$, i.e., the occurrence of HRHD is absolutely necessary to maintain transient creep. On the other hand, in transient creep of both Gr.III of MJP and Gr.III of 4W, the determinant term for creep rate changes from $-\Delta\left[Q_{s}\right]$ to $\Delta\left[V_{s}\right]+\Delta C_{s}$ with increasing strain, as shown in Tables 7 and 9 and in both data groups the degradation in strength is confirmed as seen in Figures 7 and 12. Therefore, it is certainly natural to consider for Gr.IIIa of MJP similar with the cases of Gr.III of MJP and Gr.III of 4W that we believe the existence of a transient creep range controlled by the increase in $\Delta\left[Q_{s}\right]$, which may be caused by SBSD and the sub-grain boundary hardening due to the precipitation of $\mathrm{M}_{23} \mathrm{C}_{6}$ and the Laves phase in a strain range of $\varepsilon<0.5 \%$, though the experimental evidence is not yet obtained.

Table 10. The differences between QVC for Grs.II and III at an indicated strain of P92/MJP steel and the calculated $t_{I I}$ and $t_{I I I}$ at the average temperature and stress for Gr.III. Determining term(s) for $\log \left(t_{I I I} / t_{I I}\right)$ is (are) shown in bold-italics

\begin{tabular}{cccccc}
\hline Strain $(\%)$ & 0.5 & 1 & 2 & 5 & 30.5 \\
\hline$\Delta[Q]$ & -3.81 & $\mathbf{- 0 . 4 5}$ & $\mathbf{- 6 . 5 8}$ & $\mathbf{- 1 2 . 3 6}$ & $\mathbf{- 9 . 4 8}$ \\
$-\Delta[V]$ & -0.65 & $\mathbf{- 0 . 6 3}$ & 0.49 & 1.30 & 0.90 \\
$-\Delta C$ & $\mathbf{4 . 7 8}$ & 1.02 & 6.02 & 10.94 & 8.51 \\
$\log \left(t_{I I I} / t_{I I}\right)$ & 0.32 & -0.06 & -0.07 & -0.12 & -0.07 \\
\hline
\end{tabular}

Table 11. Changes in $Q_{s}, V_{s}, C_{s}$, and $\log (\dot{\varepsilon})$ between two adjacent strains for Gr.IIIa of P92/MJP steel

\begin{tabular}{ccccc}
\hline Strain range & {$[0.5-1 \%]$} & {$[1-2 \%]$} & {$[2-5 \%]$} & {$\left[5 \%-t_{r}\right]$} \\
\hline$-\Delta\left[Q_{s}\right]$ & 1.79 & $\mathbf{5 . 8 9}$ & $\mathbf{1 . 2 3}$ & -41.33 \\
$\Delta\left[V_{s}\right]$ & $\mathbf{- 0 . 8 9}$ & -0.38 & 0.32 & $\mathbf{4 . 4 0}$ \\
$\Delta C_{s}$ & $\mathbf{- 1 . 5 2}$ & -5.41 & -1.11 & $\mathbf{3 7 . 7 2}$ \\
$\log (\dot{\varepsilon}, \% / \mathrm{h})$ & -0.63 & 0.10 & 0.45 & 0.79 \\
\hline
\end{tabular}

However, it may also be possible to think that SBSD is insufficient due to the low stresses in Gr.IIIa and HRHD begins from the initial stage of creep. In any case, it is desirable to investigate the creep behavior at low stresses 
around the conditions of Gr.IIIa. In $\varepsilon>1 \%$, the determinant term for the $\dot{\varepsilon}$ is $-\Delta\left[Q_{s}\right]$ and the values of $V$ shown in Figure 14 remain small, which indicates the occurrence of HRHD even in accelerating creep, and therefore, the decrease in number density of finely dispersed MX particles due to the formation of the Z-phase is the key to the rupture lives. Hence, the factorial analyses were performed for $t_{r}$ and $t_{\varepsilon}$ of Grs.III and IIIa under the average test conditions of Gr.IIIa adopting the same method as Table 10 and the results are shown in Table 12. During the entire duration of creep, the decrease in the activation energy, $\Delta[Q]$, is a detrimental term for the degradation of $t_{r}$ and $t_{\varepsilon}$ of Gr.IIIa, which is mainly caused by the formation of the Z-phase consuming the strengthening particles of MX.

Table 12. The differences in QVC for Grs.III and IIIa at an indicated strain of P92/MJP steel and the calculated $t_{I I I}$ and $t_{I I I a}$ at the average temperature and stress for Gr.IIIa. Determining term for $\log \left(t_{I I I a} / t_{I I I}\right)$ is shown in bold-italics

\begin{tabular}{cccccc}
\hline Strain $(\%)$ & 0.5 & 1 & 2 & 5 & 22.8 \\
\hline$\Delta[Q]$ & $\mathbf{- 2 2 . 6 0}$ & $\mathbf{- 2 1 . 1 4}$ & $\mathbf{- 1 9 . 6 3}$ & $\mathbf{- 1 5 . 9 3}$ & $\mathbf{- 1 2 . 2 0}$ \\
$-\Delta[V]$ & 2.63 & 2.71 & 2.45 & 1.98 & 1.67 \\
$-\Delta C$ & 19.60 & 18.16 & 16.97 & 13.79 & 10.38 \\
$\log \left(t_{\text {III }} / t_{\text {III }}\right)$ & -0.37 & -0.26 & -0.21 & -0.16 & -0.14 \\
\hline
\end{tabular}

\subsubsection{Gr.IV}

The values of $Q$ and $C$ for $G r . I V$ are small and similar to those of Gr.IIIa, which indicates that the microstructure is considered to be fully recovered through the entire duration of creep. However, the values of $V$ are very large $\left(\sim 1000 \mathrm{~cm}^{3} \mathrm{~mol}^{-1}\right)$, which indicates that the sub-grains are well-developed and the substructures are similar to those of Gr.IIa. The average test conditions are $704{ }^{\circ} \mathrm{C}, 31 \mathrm{MPa}, t_{0.5}=8875 \mathrm{~h}, t_{1}=21601 \mathrm{~h}, t_{2}=20970 \mathrm{~h}$, $t_{5}=47844 \mathrm{~h}$, and $t_{r}=43256 \mathrm{~h}$. The reason for $t_{5}>t_{r}$ is similar to that for the case of Gr.IIIa. The start time of the Z-phase formation at an average temperature of Gr.IV is $\sim 10000 \mathrm{~h}$ according to the TTP diagram for Grade T92 (Sawada et al., 2007) and so the $\mathrm{Z}$ phase is formed through the approximately entire duration of creep of $\varepsilon>0.5 \%$. The rupture data at $700-750{ }^{\circ} \mathrm{C}$ of $G r . I V$ are on the extrapolated lines of Gr.IIa for each, but both groups are different from each other in terms of the existence of the Z-phase. The HRHD in Gr.IV is considered to be less considerable than that of Gr.IIIa, because the values of $V$ is considerably large for Gr.IV. Even if HRHD takes place and the Z-phase is formed, the degree of deformation in the heterogeneously recovered region is not as large compared to that of Gr.IIIa, because the stress concentration is relaxed due to the growth of sub-grains. Therefore, in Gr.IV the decrease in strength due to HRHD may not so large. Moreover, the stress levels of Gr.IV are relatively closer to those in the actual power plants compared to those of Gr.IIIa. Therefore, we can expect that time to rupture of P92/MJP tested under actual operating conditions of temperatures are not as short as those extrapolated linearly from the regression lines for Gr.IIIa but are a much longer time. This is an important issue, and therefore, we will discuss further in Section 5.2 after surveying the creep behavior of similar steels such as T92/MJT and T91/MGC.

\subsection{T92/MJT}

\subsubsection{Time to Rupture and Analysis of Creep Curves}

NIMS Creep Data Sheet No. 48B (2018) contains the creep data of tubes with NIMS reference codes of MJT, MJU, and MJV. Among those heats, MJT exhibits the longest time to rupture which is $\sim 100000 \mathrm{~h}$. Figure 15 shows the $\sigma-t_{r}$ relationship for T92/MJT. The data are classified into 6 groups, Gr.I $-I V$ referring to $\sigma-t_{\varepsilon}$ and $\sigma-M C R$ diagrams (Figure A6) and Figure 12 for P92/MJP. Four data points shown by daggers in Figure 15 were not classified into any group. Regression analyses were performed according to Equation 3 and the same group names as for P92/MJP are maintained for the test conditions similar to those of MJP. In Figure 15 although a data point at $575{ }^{\circ} \mathrm{C}$ and $160 \mathrm{MPa}$ appears to be on the regression line at $575^{\circ} \mathrm{C}$ for $\mathrm{Gr} . I I I$, as shown in Figure A6a) $t_{0.5}$ under $160 \mathrm{MPa}$ is considerably shorter than that under the higher stress of $170 \mathrm{MPa}$ and thus the data was not classified into any data group. Rupture strength of T92/MJT appears to deteriorate over time according to an S-shape at temperatures $\sim 600{ }^{\circ} \mathrm{C}$ and this behavior is similar to that of P92/MJP. The break down in long-term rupture strength in Gr.IIIa is seen as a problem, but the slope in the $\sigma-\log t_{r}$ relationship for Gr.IV is gentler than that of Gr.IIIa, whereas Gr.IV is located at a lower stress and longer time region than that of Gr.IIIa as seen in Figure 15. In the bottom right region of the spool line in Figure 15, $R A<70 \%$ like in Figure 12 
and crosses in the figure denote $R A=0 \%$, although the $E L \mathrm{~s}$ at these conditions are $\sim 10 \%$ and moreover, $R A$ tends to recover after a period of time. The spool line of MJT in Figure 15 shifts to a shorter time area compared to that of MJP (Figure 12). Though the figures are omitted, a spool line for $R A=70 \%$ of T92/MJV is $\sim 10000 \mathrm{~h}$ and is not so largely dependent on temperature and that for MJU lies between those for MJP and MJT. Though the pre-existing BN particles are considered to be a major factor for the low ductility in Grade 92 (Gu et al., 2014; Parker \& Siefert, 2018; Abe et al., 2018), the above mentioned facts indicate that factors other than BN affect the low ductility of Grade 92. Normalizing temperature, Hv, C and Ni contents for MJU are $1050{ }^{\circ} \mathrm{C}, 226,0.10 \%$, and $0.27 \%$, respectively, and $1050{ }^{\circ} \mathrm{C}, 234,0.092 \%$, and $0.15 \%$ for MJV, respectively. The decrease in ductility of Grade 92 may be related to these factors in addition to the amount of BN, but there are insufficient data to further discuss the decrease in ductility of Grade 92.

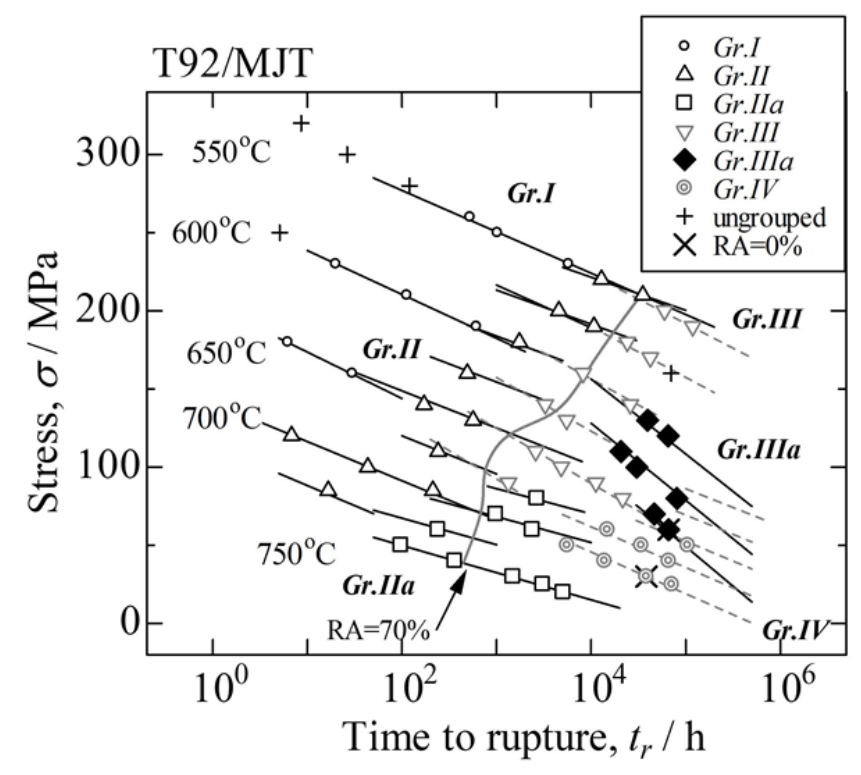

Figure 15. The stress vs. time to rupture relationship for T92/MJT and regression lines for each data group explained in Section 4.4.1. RAs for the bottom right of a spool line are below $70 \%$

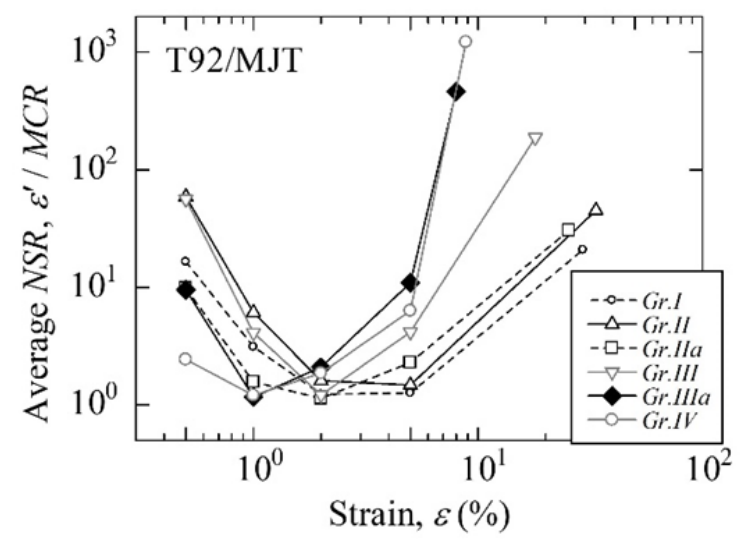

Figure 16. The average NSR vs. strain relationship for T92/MJT

Several abnormal data such as $t_{\varepsilon}$ at a lower stress is shorter than that at higher stress are confirmed in $\sigma-t_{\varepsilon}$ diagrams at $550-675{ }^{\circ} \mathrm{C}$ and $\varepsilon=0.5$ and $1 \%$ for T92/MJT as shown in Figures A6a) and b), although abnormal data like these were not observed during accelerating creep in the $\sigma-t_{\varepsilon}$ diagrams for MJT. These phenomena were not confirmed for MJP, MJU, and MJV of Grade 92, but abnormal data like these were also confirmed for reference code MGB and MgC of Grade 91 (NIMS, 2007). Therefore, this phenomenon is not unique, but there must be reasons. However, the phenomenon has not yet been studied. 
Figure 16 shows the strain vs. average NSR relationship for MJT. The changes in average NSR for MJT are qualitatively similar to that of MJP shown in Figure 13. The rapid strain hardening during transient creep of Gr.IIIa triggers the subsequent increase in creep rate during accelerating creep which leads to the break down in rupture strength. Figure 17 shows variations of $Q V C$ as functions of strain for each group. The Z-phase is formed in Grs.III, IIIa, and IV. The values of $Q$ at rupture for Grs.IIIa and $I V$ where the break down in rupture strength is observed in Figure 15 are $\sim 500 \mathrm{kJmol}^{-1}$ and are larger than the activation energy for self-diffusion of alpha-iron. However, the values of $C$ are $\sim 20$, which are similar values confirmed in many soft-type heat-resistant steels (Tamura et al., 2013). On the other hand, $Q>600 \mathrm{kJmol}^{-1}$ and $C>30$ at rupture are obtained for other groups, which means that the hard microstructure is maintained until rupture.
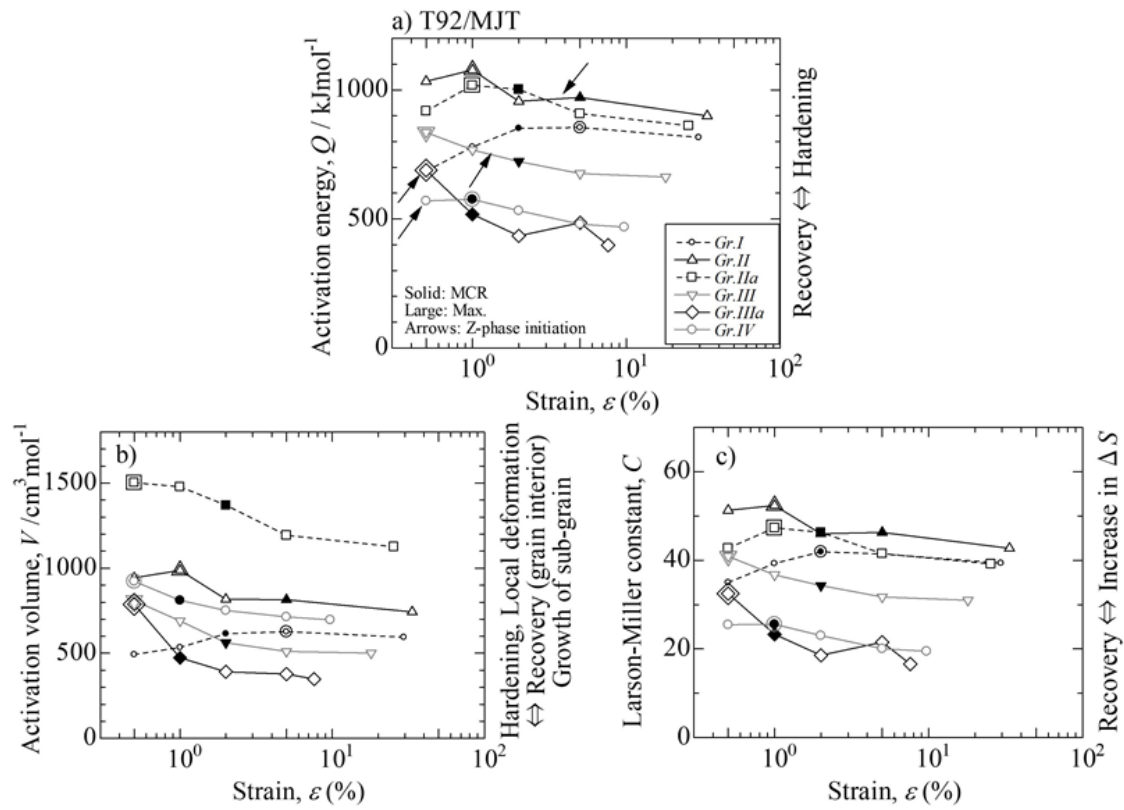

Figure 17. The results of the $Q V C$ analysis are shown as functions of creep strain for each group of T92/MJT steel; a) the activation energy, b) the activation volume, and c) the Larson-Miller constant

\subsubsection{Gr.I}

The average test conditions are $594{ }^{\circ} \mathrm{C}, 217 \mathrm{MPa}, t_{0.5}=319 \mathrm{~h}$, and $t_{r}=12377 \mathrm{~h}$. The Laves phase is not formed under the average test conditions for Gr.I (Haetterstrand \& Andren, 2001; Zielinski et al., 2016). The $Q$ and $V$ increase up to $\varepsilon=2 \%$, which is a little different from that of Gr.I of MJP shown in Figure 14. This initial increase in $Q$ and $V$ is caused by SBSD and the $Q$ for MJT is larger than that of MJP (Figure 14), because initial Hv in MJT is higher than that of MJP shown in Table 3, and thus many more dislocations are swept out to the sub-boundaries. After $\varepsilon>2 \%, Q V C$ slightly decrease and thus the microstructure recovers at a slow pace during creep thus maintaining the martensite lath structure with $\mathrm{M}_{23} \mathrm{C}_{6}$ and fine MX particles.

\subsubsection{Gr.II}

The average test conditions for Gr.II are $637{ }^{\circ} \mathrm{C}, 148 \mathrm{MPa}, t_{1}=174 \mathrm{~h}, t_{2}=1297 \mathrm{~h}, t_{5}=4608 \mathrm{~h}$, and $t_{r}=5136 \mathrm{~h}$. Up to $1 \%$ strain, all of $Q V C$ increase slightly, which demonstrates that SBSD occurs. However, the values of $Q$ are larger than those for Gr.II of MJP and so, sub-boundary strengthening by the Laves phase together with $\mathrm{M}_{23} \mathrm{C}_{6}$ occurs. Kipelova et al. (2012) showed that the Laves phase precipitates on the lath boundaries in a similar steel, P911(3Co) crept to $1 \%$ strain $(374 \mathrm{~h})$ at $650{ }^{\circ} \mathrm{C}$ and therefore, the Laves phase possibly precipitates under the conditions of Gr.II up to $1 \%$ strain. Peak time of $Q$ for MJT $(174 \mathrm{~h})$, is much shorter than that of MJP $(1015 \mathrm{~h})$, which is caused by the promotion of precipitation of the Laves phase due to the higher content of Si in MJT compared with that of MJP, as shown in Table 3 (Hosoi et al., 1986). In $\varepsilon>1 \%$, both $Q$ and $V$ decrease slightly, which indicates that HRHD and recovery due to the coarsening of the Laves phase begin to occur. The start time for Z-phase formation is $\sim 3700 \mathrm{~h}$ at a temperature of that of Gr.II (Sawada et al., 2007) and thus there may be slight Z-phase formation during accelerating creep. However, the value of $Q$ at 
rupture still maintains high values of $\sim 900 \mathrm{kJmol}^{-1}$ and therefore the strengthening effects based on the martensite structure with MX, Laves phase, and $\mathrm{M}_{23} \mathrm{C}_{6}$ are retained until rupture and are far superior to the negative effect of the Z-phase.

\subsubsection{Gr.IIa}

The average test conditions for Gr.IIa are $728^{\circ} \mathrm{C}, 48 \mathrm{MPa}, t_{1}=378 \mathrm{~h}, t_{2}=1297 \mathrm{~h}$, and $t_{r}=1817 \mathrm{~h}$. The $Q$ and $C$ increase up to $\varepsilon=1 \%$, and reach $Q \sim 1018 \mathrm{kJmol}^{-1}$ and $C \sim 47.3$, respectively, before decreasing. The $V$ peaks at $1479 \mathrm{~cm}^{3} \mathrm{~mol}^{-1}$ at $\varepsilon=0.5 \%$ and then decreases to $\sim 1100 \mathrm{~cm}^{3} \mathrm{~mol}^{-1}$. Creep behavior of Gr.IIa is very similar to that of Gr. II, but the Z-phase is not formed throughout the entire duration. The SBSD mechanism operates at the early stage of transient creep, followed by HRHD accompanying the strengthening of sub-boundaries by $\mathrm{M}_{23} \mathrm{C}_{6}$ and the Laves phase, but there is only moderate recovery of the martensite structure and coarsening of $\mathrm{M}_{23} \mathrm{C}_{6}$ and the Laves phase. The high values of $Q \sim 862 \mathrm{kJmol}^{-1}$ and $C \sim 49.2$ hold at rupture, and therefore, a high level of back stress is maintained throughout the duration of creep without consuming the finely dispersed MX particles.

\subsubsection{Gr.III}

The average test conditions for Gr.III are $613{ }^{\circ} \mathrm{C}, 137 \mathrm{MPa}, t_{0.5}=220 \mathrm{~h}, t_{1}=1990 \mathrm{~h}, t_{2}=14734 \mathrm{~h}$, and $t_{r}=25317 \mathrm{~h}$. All of the QVC decrease through the entire duration. This trend is different from that of P92/MJP, where the peaks of $Q V C$ are observed at $\varepsilon=1 \%$. The high value of $Q$ at $\varepsilon=0.5 \%$ is caused by the formation of the Laves phase (Kipelova et al., 2012) and SBSD. Immediately following the HRHD occurs up to $\varepsilon=5 \%$ or perhaps up to rupture. The Z-phase begins to form between $\varepsilon=1-2 \%$ ( $4000 \mathrm{~h}$ ) (Sawada et al., 2007), which leads to the degradation in strength compared to that of Gr.II. However, $Q \sim 700 \mathrm{kJmol}^{-1}$ at rupture is confirmed, and so the hard microstructure is considered to be maintained up to rupture.

\subsubsection{Gr.IIIa}

The average test conditions for Gr.IIIa are $622{ }^{\circ} \mathrm{C}, 96 \mathrm{MPa}, t_{0.5}=4815 \mathrm{~h}, t_{1}=27220 \mathrm{~h}, t_{2}=50038 \mathrm{~h}$, $t_{5}=41300 \mathrm{~h}$ and $t_{r}=49878 \mathrm{~h}$. One of the data in Gr.IIIa at $\varepsilon=5 \%$ is lacking resulting in $t_{5}<t_{2}$. The $Q V C$ vs. strain variations for MJT are similar to those of MJP and the values of $Q V C$ are the smallest except for $\varepsilon=0.5 \%$. After $\varepsilon=1 \%, Q \sim 450 \mathrm{kJmol}^{-1}, V \sim 400 \mathrm{~cm}^{3} \mathrm{~mol}^{-1}$ and $C \sim 20$, which indicates that microstructure is fully recovered and HRHD is continuing. Z-phase is confirmed at least at $\varepsilon=0.5 \%(4300 \mathrm{~h})$ (Sawada et al., 2007). Therefore, though the SBSD and subsequent sub-boundary strengthening by $\mathrm{M}_{23} \mathrm{C}_{6}$ and Laves phase occurs at an early stage of transient creep, microstructure is reorganized through both the decomposition of martensite structure through HRHD and the formation of Z-phase which consumes the strengthening particles of MX in accelerating creep. The $Q_{s}, V_{s}$, and $C_{s}$ analyses were also made on Gr.IIIa. It was confirmed as the results for MJP shown in Table 11 that in a transient creep, $\varepsilon \leq 1 \%$, the decrease in $\Delta\left[V_{s}\right]+\Delta C_{s}$ is the determinant terms, even though $\Delta\left[Q_{s}\right]$ decreases due to the coarsening of precipitates and the formation of Z-phase, though the table is omitted. Therefore, the decrease in $\Delta\left[V_{s}\right]$, i.e., HRHD is absolutely necessary to keep transient creep. As shown in Figure A6, $t_{\varepsilon} \mathrm{s}$ for Gr.IIIa become shorter than those for Gr.III in $\varepsilon>1 \%$. Factorial analyses similar with Table 12 for MJP were made on Grs.III and IIIa. It was found that in $\varepsilon>1 \%$ the decrease in $Q$ mainly caused by the formation of Z-phase is the determinant term for the degradation in strength, though the table were omitted.

\subsubsection{Gr.IV}

The average test conditions for Gr.IV are $684{ }^{\circ} \mathrm{C}, 43 \mathrm{MPa}, t_{0.5}=6876 \mathrm{~h}, t_{1}=17868 \mathrm{~h}, t_{2}=30556 \mathrm{~h}$, $t_{5}=42670 \mathrm{~h}$, and $t_{r}=43633 \mathrm{~h}$. The values of $Q$ and $C$ throughout the duration of creep are higher than those of Gr.IIIa. According to previous work (Sawada et al., 2007) the Z-phase also forms throughout the duration of creep of $\varepsilon>0.5 \%$. This indicates that the microstructure is fully recovered consuming the finely dispersed MX particles. But, the values of $V$ are considerably larger than those for Gr.IIIa. This indicates that sub-grains grow considerably large and hence, the degree of HRHD is reduced. Therefore, the rupture strength of Gr.IV is increased or improved compared to the strength estimated using the data of Gr.IIIa as shown in Figure 15.

\section{$4.5 T 91 / M G C$}

\subsubsection{Time to Rupture and Analysis of Creep Curves}

NIMS $(2007 ; 2014)$ published the digital creep data of tubes, pipes, and plates of Grade 91. Among these, the creep data of Grade T91/MGC was selected and analyzed, because both the long-term creep data and the results of metallurgical investigation are available for this heat, provided that the creep data at $600{ }^{\circ} \mathrm{C}$ and $70 \mathrm{MPa}$ were read from $t-\dot{\varepsilon}$ and $t-\varepsilon$ diagrams published in the previous work (Sawada et al., 2011; Kimura, Sawada, Kushima, \& Toda, 2012). Figure 18 shows the $\sigma-t_{r}$ relationship for T91/MGC. The creep data were classified 
into 7 groups, Grs.I - IV referring to the $\sigma-t_{\varepsilon, M C R}$ relationships for T91/MGC. Figure A7 shows $\sigma-$ $t_{0.2}, t_{0.5}$, and $t_{1}$ relationships as examples, however in these figures the regression lines for Gr.IV are lacked, because the creep curve is only obtained at $725^{\circ} \mathrm{C}$ and $40 \mathrm{MPa}$. A data point at $500{ }^{\circ} \mathrm{C}$ and $340 \mathrm{MPa}$ was not classified into any group. Abnormal creep data as shown in Figure A6 for T92/MJT is not found as that for T91/MGC. The $t_{r}$ changes in the S-shape at $625^{\circ} \mathrm{C}$, but at other temperatures the rupture strength decreases monotonically with increasing $t_{r}$. Test conditions where the break down in strength is a critical issue correspond to Grs.IIIa and IIIb (Kushima et al., 1999; Sawada et al., 2011). The reduction in ductility as shown in Figures 12 and 15 for Grade 92 is not observed for T91/MGC. Figure 19 shows the $\varepsilon$ - average NSR relationships for Grs.I-IV, provided that the number of data for both Grs.IIIb and $I V$ are only 1.

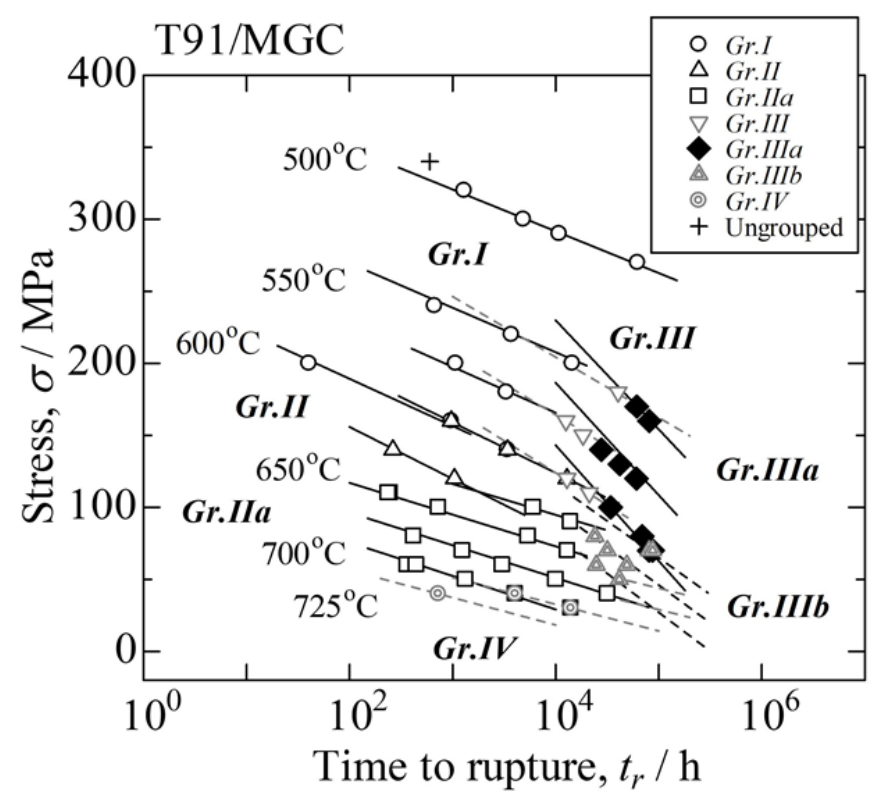

Figure 18. Stress vs. time to rupture relationship for T91/MGC and regression lines for each data group explained in Section 4.5.1

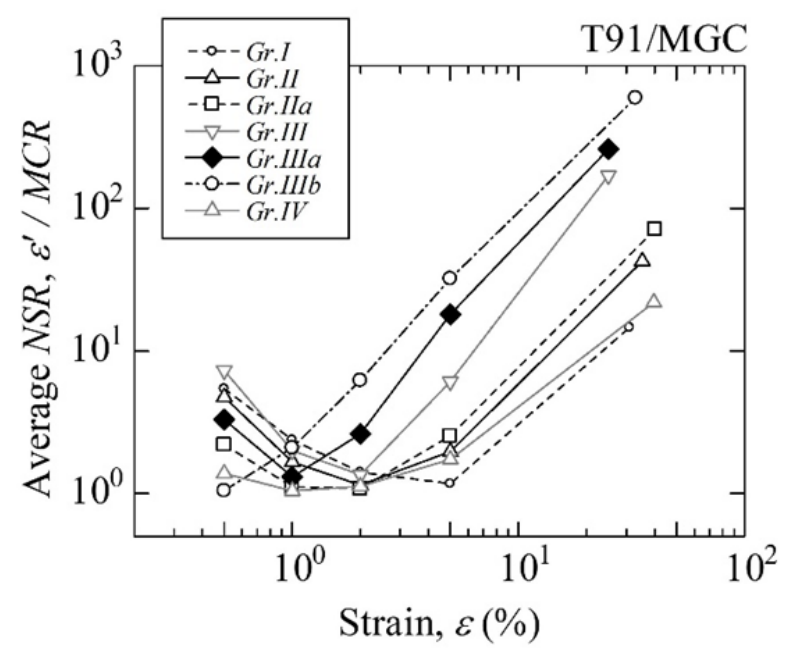

Figure 19. The average NSR vs. strain relationship for T91/MGC

The changes in the average NSR for MGC shown in Figure 19 are qualitatively similar to that of MJP and MJT 
shown in Figures 13 and 16. The rapid strain hardening in transient creep for Gr.IIIa triggers the subsequent increase in the creep rate during accelerating creep which leads to the break down in rupture strength, although transient creep is not observed for Gr.IIIb. Figure 20 shows the variations of $Q V C$ as functions of strain for each group, provided that the $Q V C$ s during creep for Grs.IIIb and $I V$ were not obtained because of insufficient number of data. The Z-phase is formed during the entire duration of creep for Grs.III, IIIa, and IIIb. The values of $Q$ at rupture for Grs.IIIa and IIIb where the break down in rupture strength is observed (Figure 18) are lower than $500 \mathrm{kJmol}^{-1}$ and are larger than the activation energy for self-diffusion of alpha-iron, but the values of $C$ are approximately $<20$ and particularly $C$ for $G r . I I I b$ is extremely low. On the other hand, $Q>600 \mathrm{kJmol}^{-1}$ and $C>30$ are obtained for Grs. I, II, and IIa at rupture, which means that the hard microstructure is maintained until rupture.
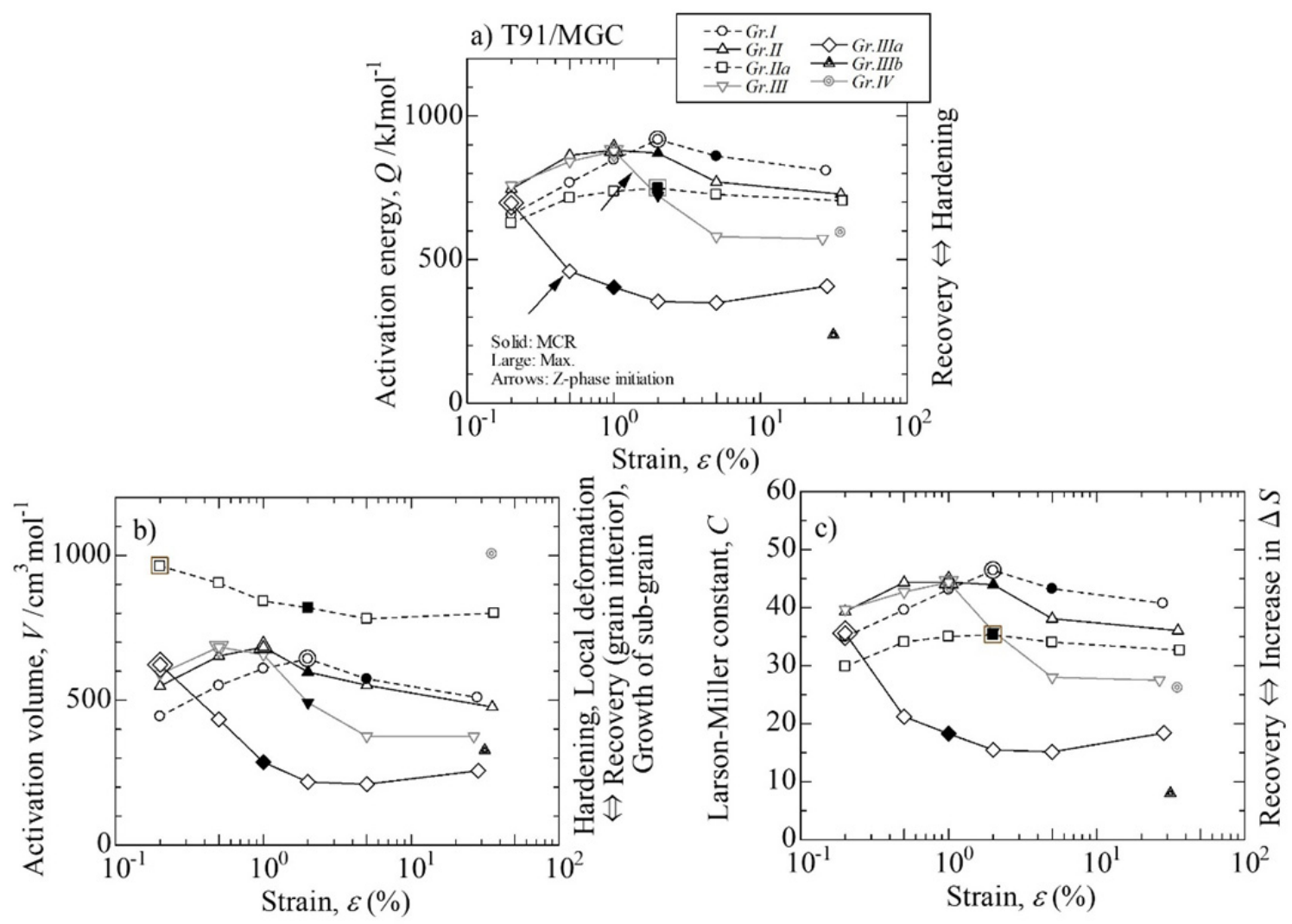

Figure 20. The results of the $Q V C$ analysis are shown as functions of creep strain for each group of T91/MGC steel; a) the activation energy, b) the activation volume, and c) the Larson-Miller constant

The creep data at $600{ }^{\circ} \mathrm{C}$ and $70 \mathrm{MPa}$ belongs to Gr.IIIa and the time vs. strain relationship used in Figures 19, 20 , and $\mathrm{A} 7$ is converted essentially from the $t-\dot{\varepsilon}$ diagram and the $t-\varepsilon$ diagram in the previous work (Sawada et al., 2011; Kimura et al., 2012). The relevant problems on the accuracy of the creep data for Gr.IIIa at $600{ }^{\circ} \mathrm{C}$ and $70 \mathrm{MPa}$ will be discussed in Sections 4.5.6 and 5.2.3.

\subsubsection{Gr.I}

The average test conditions are $575^{\circ} \mathrm{C}, 193 \mathrm{MPa}, t_{2}=1406 \mathrm{~h}$, and $t_{r}=3891 \mathrm{~h}$. The $Q$ and $V$ increase up to $\varepsilon=2 \%$, and then decrease. The Z-phase is not formed for Gr.I. The initial increase in $Q$ and $V$ is caused by SBSD and grain boundary strengthening due to $\mathrm{M}_{23} \mathrm{C}_{6}$ similar to those of each Gr.I of MJP and MJT. However, the peak value of $Q=917.7 \mathrm{kJmol}^{-1}$ of MGC is higher than $Q=806.1$ and $854.7 \mathrm{kJmol}^{-1}$ for Grs.I of P92/MJP and MJT, respectively. The main reason for this may arise from the difference in initial hardness (see Table 3), however it cannot be denied that the precipitation of the Laves phase assists the grain boundary strengthening in transient creep of MGC. The reason for this is that the nose point of the TTP curve for the Laves phase in an equivalent Grade 91 steel is at $575{ }^{\circ} \mathrm{C}$ for $1700 \mathrm{~h}$ (Tamura et al., 1988) and this nose 
temperature for $\mathrm{Fe}_{2} \mathrm{Mo}$ is just the same as the average temperature of Gr.I. Moreover, precipitation is generally promoted by creep strain and therefore, the Laves phase strengthening may be possible even in transient creep for Gr.I of MGC, $\varepsilon<2 \%$. After $\varepsilon>2 \%$, the QVC gradually decrease due to recovery and coarsening of precipitates except MX. However, MCR occurs at $\varepsilon=5 \%$, and therefore HRHD should be in progress between $2-5 \%$ strain.

\subsubsection{Gr.II}

The average test conditions are $610{ }^{\circ} \mathrm{C}, 136 \mathrm{MPa}, t_{1}=830 \mathrm{~h}, t_{2}=2198 \mathrm{~h}, t_{5}=3392 \mathrm{~h}$ and $t_{r}=3709 \mathrm{~h}$. The $Q$ and $V$ increase up to $\varepsilon=1 \%$, and then decrease. The initial increase in $Q$ and $V$ is caused by SBSD and grain boundary strengthening due to $\mathrm{M}_{23} \mathrm{C}_{6}$ and the Laves phase like the cases for Gr.II of MJP and MJT, because the Laves phase is confirmed in a specimen of this steel ruptured at $600^{\circ} \mathrm{C}$ for $40.4 \mathrm{~h}$ by Suzuki et al. (2000). The QVC behavior along the strain axis of Gr.II is similar to the compressed form of the behavior for Gr.I, and therefore, it is reasonable to consider that in Gr.II the creep phenomenon of Gr.I occurs in a shorter time by increasing the temperature and that the HRHD should occur between $1-2 \%$ strain. In accelerating creep, recovery of the martensite structure and coarsening of $\mathrm{M}_{23} \mathrm{C}_{6}$ and the Laves phase occur moderately, because the start time for the Z-phase at $610{ }^{\circ} \mathrm{C}$ is approximately $6200 \mathrm{~h}$ (Sawada et al., 2007) and thus the Z-phase is not formed through the entire duration until rupture and the strengthening particles of $\mathrm{XM}$ are not consumed. The average temperature for Gr.II of MGC is $\sim 30{ }^{\circ} \mathrm{C}$ lower than those for each Gr.II of MJP and MJT. This may result from the fact that the nose temperature of the TTP curve of $\mathrm{Fe}_{2} \mathrm{Mo}$ is lower than that of $\mathrm{Fe}_{2} \mathrm{~W}$ (Tamura et al., 1988). On the other hand, the maximum value of $Q=881.3 \mathrm{kJmol}^{-1}$ for Gr.II of MGC is smaller than those for MJP and MJT, $Q \sim 1000 \mathrm{kJmol}^{-1}$. This may arise because the amount of Laves phase precipitated for MGC is estimated to be less than those for MJP and MJT which is inferred from the total amount of Mo and W for each steel. Therefore, the strengthening effect in T91/MGC is not as great as in Gr.II.

\subsubsection{Gr.IIa}

The average test conditions are $663{ }^{\circ} \mathrm{C}, 77.5 \mathrm{MPa}, t_{2}=2598 \mathrm{~h}$ and $t_{r}=4428 \mathrm{~h}$. The $M C R$ occurs at $2 \%$ strain. The Z-phase does not form during creep (Suzuki et al., 2000). The $Q$ and $C$ increase slightly up to $\varepsilon=2 \%$, and then decrease. $V \sim 1000 \mathrm{~cm}^{3} \mathrm{~mol}^{-1}$ is already attained at $\varepsilon=0.2 \%$, and then decreases. The high value of $V$ indicates that a highly recovered state with the growth of sub-grains continues through the entire duration of creep. However, up to $2 \%$ strain of transient creep the $Q$ and $C$ increase and the $V$ decreases, which shows that HRHD with the DFRP mechanism for $\mathrm{M}_{23} \mathrm{C}_{6}$ occurs. The $Q_{s}, V_{s}$, and $C_{s}$ analyses showed that the creep rate is controlled by the increase in $\left[Q_{s}\right]$ up to $\varepsilon=0.5 \%$, the increase in $\left[Q_{s}\right]$ and the decrease in $\left[V_{s}\right]$ between $\varepsilon=0.5-2.0 \%$, and the decrease in $\left[Q_{s}\right]$ in accelerating creep, although the table is omitted. Therefore, to maintain transient creep a decrease in $V$ or the occurrence of HRHD is the necessary condition.

\subsubsection{Gr.III}

The average test conditions are $580{ }^{\circ} \mathrm{C}, 142 \mathrm{MPa}, t_{0.2}=79.6 \mathrm{~h}, t_{0.5}=1614 \mathrm{~h}, t_{1}=7788 \mathrm{~h}, t_{2}=17420 \mathrm{~h}$, and $t_{r}=24613 \mathrm{~h}$. Z-phase begins to form at approximately $13500 \mathrm{~h}$, i.e., between $t_{1}-t_{2}$. The $M C R$ occurs at $\varepsilon \sim 2 \%$. The $Q V C$ values increase up to $\sim 1 \%$ strain and then decrease largely for each. Laves phase precipitate between $t_{0.2}-t_{0.5}$ (Tamura et al., 1988). The SBSD with the DFRP of $\mathrm{M}_{23} \mathrm{C}_{6}$ operates up to $\varepsilon=0.2 \%$ and then grain boundary strengthening by Laves phase is added for $\varepsilon=0.2-0.5 \%$. Looking at around the peaks of $Q V C$ in detail, the $V$ begins to decrease at $\varepsilon \sim 0.5 \%$, though the $Q$ and $C$ are still increasing up to $\sim 1 \%$. Therefore, HRHD occurs at least $\varepsilon=0.5-1 \%$ in transient creep accompanying the DFRP mechanism for $\mathrm{M}_{23} \mathrm{C}_{6}$ and grain boundary strengthening by Laves phase, and the HRHD continues with the decreases in $Q$ and $C$ until rupture passing through the $M C R$ point. Table 13 shows the results of the $Q_{s}$, $V_{s}$, and $C_{s}$ analyses for Gr.III. The table shows that the determinant term for the creep rate is $\left[Q_{s}\right]$ through the whole duration of creep, but $-\left[Q_{S}\right]+\left[V_{s}\right]$ is the determinant terms for $\varepsilon=0.5-1 \%$ in transient creep. Therefore, the decrease in $\left[V_{s}\right]$, i.e., the occurrence of HRHD, is essentially necessary to keep transient creep between $\varepsilon=0.5-1 \%$. Although Figure 19 shows that $M C R$ occurs at $\varepsilon=2 \%$, Table 13 shows that an accelerating creep begins between $\varepsilon=1-2 \%$. In this strain range all of the $Q V C$ decrease and Z-phase is possibly formed, and therefore, recovery accompanying HRHD begins and is continuing up to rupture accompanying the growth of sub-grains, coarsening of precipitates, and consuming some of MX particles. Time to ruptures in Gr.III are inferior to those estimated from Gr.II. This can be explained from the degree of recovery during accelerating creep, i.e., the decrease in $Q$ of Gr.III is larger than that of Gr.II which is promoted by the occurrence of the HRHD behavior due to the lower values of $V$. 
Table 13. Changes in $Q_{s}, V_{s}, C_{s}$, and $\log (\dot{\varepsilon})$ between two adjacent strains for Gr.III of T91/MGC steel

\begin{tabular}{cccccc}
\hline Strain range & {$[0.2-0.5 \%]$} & {$[0.5-1 \%]$} & {$[1-2 \%]$} & {$[2-5 \%]$} & {$\left[5 \%-t_{r}\right]$} \\
\hline$-\Delta\left[Q_{s}\right]$ & $-\mathbf{4 . 9 5}$ & $\mathbf{- 0 . 8 0}$ & $\mathbf{1 3 . 8 8}$ & $\mathbf{2 2 . 8 6}$ & $\mathbf{1 . 7 8}$ \\
$\Delta\left[V_{s}\right]$ & 1.15 & $\mathbf{- 0 . 4 6}$ & -1.97 & -2.33 & 0.20 \\
$\Delta C_{s}$ & 3.04 & 0.81 & -11.89 & -19.83 & -0.56 \\
$\log (\dot{\varepsilon}, \% / \mathrm{h})$ & -0.76 & -0.45 & 0.02 & 0.70 & 1.42 \\
\hline
\end{tabular}

\subsubsection{Gr.IIIIa}

The average test conditions of Gr.IIIa are $581^{\circ} \mathrm{C}, 116 \mathrm{MPa}, t_{0.2}=1260 \mathrm{~h}, t_{0.5}=12377 \mathrm{~h}, t_{1}=29529 \mathrm{~h}$, $t_{2}=47705$, and $t_{r}=58699 \mathrm{~h} . M C R$ occurs at $\varepsilon \sim 1 \%$. The start time of the Z-phase formation is approximately $13500 \mathrm{~h}$ at the average temperature corresponding to $\sim 0.5 \%$ strain (Sawada et al., 2007; 2011) and the size of the Z-phase is rather small, $\sim 50 \mathrm{~nm}$ in a ruptured specimen at $600{ }^{\circ} \mathrm{C}$ for $10000 \mathrm{~h}$ just above the average temperature of Gr.IIIa. On the other hand, the Laves phase is coarsened up to $7700 \mathrm{~nm}$ for these conditions (Suzuki et al., 2000). The QVC values at $\varepsilon=0.2 \%$ are already considerably large, $697.2 \mathrm{kJmol}^{-1}$, $622.4 \mathrm{~cm}^{3} \mathrm{~mol}^{-1}$, and 35.62 , respectively. These values decrease rapidly with an increase in strain up to $1 \%$ and then remain roughly constant at $\sim 400 \mathrm{kJmol}^{-1}, 200 \mathrm{~cm}^{3} \mathrm{~mol}^{-1}$, and 18 up to rupture, respectively. The break down in rupture strength occurs in Gr.IIIa. This breakdown in strength is already observed at $t_{0.5}$ as shown in Figure A7b). The results of the $Q_{s}, V_{s}$, and $C_{s}$ analyses for Gr.IIIa shown in Table 14 indicate that $\left[V_{s}\right]+C_{s}$ are the determinant terms for the strain rate up to $\varepsilon=1 \%$ of transient creep. On the other hand, in the later part of transient creep for Gr.III, $-\left[Q_{s}\right]+\left[V_{s}\right]$ was the determinant term, as shown in Table 13. This point for Gr.IIIa is different from Gr.III, however, either way $\left[V_{s}\right]$ is the key determinant term. Namely, the HRHD takes place and the Laves phase near the heterogeneously recovered zone is coarsened remarkably. After $\varepsilon=0.5 \%$, the Z-phase is formed consuming some of the MX particles and consequently fully recovered zones are formed, which leads to a decrease in $C_{s}$. As a result, the creep strength begins to decline dramatically from $\varepsilon=0.5 \%$ as shown in Figure A7b).

In accelerating creep, a decrease in $\left[Q_{S}\right]$ is essentially the determinant term and consequently the breakdown in rupture strength becomes prominent in Gr.IIIa as seen in Figure 18. However, in the range of $\varepsilon=2-5 \%$ of accelerating creep both $\left[Q_{s}\right]$ and $C_{s}$ increase with increasing strain and thus $C_{s}$ is the determinant term. Similar phenomena are frequently observed in the $Q_{s}, V_{S}$, and $C_{s}$ analyses near rupture, but since these data contain the effect of necking, these data are not included in this discussion. However, that $C_{s}$ is the determinant term in accelerating creep without necking shown in the range of $\varepsilon=2-5 \%$ of Table 14 , is also experienced in Gr.III of $9 \mathrm{Cr}-4 \mathrm{~W}$ steel (in the range of $\varepsilon=5-10 \%$ of Table 7). Therefore, further metallurgical investigation is necessary to investigate the increase in $C_{s}$ during accelerating creep.

Table 14. Changes in $Q_{s}, V_{s}, C_{s}$, and $\log (\dot{\varepsilon})$ between two adjacent strains for Gr.IIIa of T91/MGC steel

\begin{tabular}{cccccc}
\hline Strain range & {$[0.2-0.5 \%]$} & {$[0.5-1 \%]$} & {$[1-2 \%]$} & {$[2-5 \%]$} & {$\left[5 \%-t_{r}\right]$} \\
\hline$-\Delta\left[Q_{S}\right]$ & 17.09 & 9.94 & $\mathbf{5 . 5 2}$ & -6.39 & $\mathbf{1 1 . 5 5}$ \\
$\Delta\left[V_{S}\right]$ & $\mathbf{- 2 . 2 7}$ & $\mathbf{- 2 . 1 6}$ & -0.88 & $\mathbf{0 . 2 9}$ & -0.91 \\
$\Delta C_{S}$ & $\mathbf{- 1 5 . 9 1}$ & $\mathbf{- 8 . 0 2}$ & -4.24 & $\mathbf{6 . 9 7}$ & -9.40 \\
$\log (\dot{\varepsilon}, \% / \mathrm{h})$ & -1.08 & -0.23 & 0.40 & 0.88 & 1.24 \\
\hline
\end{tabular}

Table 15. Factorial analysis for $t_{r}$ and $t_{\varepsilon}$ of Grs.III and IIIa under the average test conditions for Gr.IIIa of T91/MGC steel $\left(581^{\circ} \mathrm{C}, 116 \mathrm{MPa}\right)$ and the detrimental terms for $\log \left(t_{I I I} / t_{I I I}\right)$ are shown in bold-italics

\begin{tabular}{ccccccc}
\hline Strain $(\%)$ & 0.2 & 0.5 & 1 & 2 & 5 & 25.8 \\
\hline$\Delta[Q]$ & -3.74 & $\mathbf{- 2 3 . 4 3}$ & $\mathbf{- 2 9 . 0 4}$ & $\mathbf{- 2 2 . 5 9}$ & $\mathbf{- 1 4 . 1 6}$ & $\mathbf{- 1 3 . 9 4}$ \\
$-\Delta[V]$ & -0.23 & 1.77 & 2.63 & 1.94 & 1.17 & 1.18 \\
$-\Delta C$ & 4.05 & 21.48 & 26.11 & 20.40 & 12.84 & 12.62 \\
$\log \left(t_{\text {IIIa }} / t_{\text {III }}\right)$ & 0.08 & -0.18 & -0.30 & -0.25 & -0.15 & -0.13 \\
\hline
\end{tabular}


Again, Table 15 shows the results of the factorial analysis on the difference in creep strength between Grs.III and IIIa. It is found from the table that during the entire duration of creep (except for $\varepsilon=0.2 \%$ ), the decrease in $Q$ is the determinant factor is caused by HRHD and the consequent coarsening of the nearby precipitates and consumption of some MX particles. The above considerations coincide with the previous observations (Kushima et al., 1999; Kimura et al., 2002; Suzuki et al., 2003, Sawada et al., 2011).

The $t_{\varepsilon} \mathrm{s}$ at $600{ }^{\circ} \mathrm{C}$ and $70 \mathrm{MPa}$ for Gr.IIIa shown in Figure A7 are essentially calculated based on the $t-\dot{\varepsilon}$ diagram shown in the literature as explained in Section 4.5.1. However, the $t_{\varepsilon} \mathrm{s}$ calculated based on the $t-\varepsilon$ diagram seems to be shorter, especially for $t_{0.2}$ and $t_{0.5}$, than those calculated based on the $t-\dot{\varepsilon}$ diagram, although the reading error for smaller strains in a $t-\varepsilon$ diagram are inevitable. If we use the $t_{\varepsilon} \mathrm{s}$ calculated based on the $t-\varepsilon$ diagram instead, the slopes of the regression line for Gr.IIIa at $600{ }^{\circ} \mathrm{C}$ might be steeper than that shown in Figure A7 and therefore the values of QVC for $\varepsilon=0.2$ and $0.5 \%$ might be smaller than those shown in Figure 20. In such cases, signs for the degradation in long term rupture strength for Gr.IIIa may be recognized within a further shorter duration of time.

\subsubsection{Gr,IIIlb}

In Figure 18, Gr.IIIb is clearly different from Gr.IIIa, but the $t_{\varepsilon}$ data are not reported. The average test condition is $625^{\circ} \mathrm{C}$ and $66 \mathrm{MPa}\left(t_{r}=48547 \mathrm{~h}\right)$ and Gr.IIIb is positioned at a higher temperature and lower stress site than those for Gr.IIIa. The $M C R$ point is at $\varepsilon=1 \%$. The $Q V C$ values at rupture are $237.8 \mathrm{kJmol}^{-1}$, $327.2 \mathrm{~cm}^{3} \mathrm{~mol}^{-1}$, and 7.97 respectively. The value of $Q$ is slightly smaller than the activation energy for the self-diffusion of $\alpha$-Fe and the value of $V$ is comparable to that for Gr.IIIa. However, the value of $C$ is extremely low as heat-resistant steel and comparable to that for the notch-creep test of Grade 91 (Sikka et al., 1983). The start time of Z-phase formation is approximately $4000 \mathrm{~h}$ at the average temperature and the $t_{r}$ is affected by the Z-phase. Therefore, referring to the low values of $Q$ and $C$, the microstructure in a gauge portion at rupture must be recovered completely. However, the value of $V$ is slightly larger than that for that of Gr.IIIa. Therefore, the degree of HRHD is not so remarkable as compared to that of Gr.IIIa and thus, the breakdown in strength tends to be moderated.

\subsubsection{Gr.IV}

The average test condition is $725^{\circ} \mathrm{C}$ and $40 \mathrm{MPa}\left(t_{r}=6800 \mathrm{~h}\right)$ and $G r . I V$ is positioned at a higher temperature and lower stress site than Grs.IIa and IIIa. The MCR point is near $\varepsilon=1 \%$. The QVC values at rupture are $594.8 \mathrm{kJmol}^{-1}, 1005.3 \mathrm{~cm}^{3} \mathrm{~mol}^{-1}$, and 26.20 , respectively. The $t_{\varepsilon}$ data are also not reported like Gr.IIIb. In Gr.IV neither the Laves phase nor the Z-phase is formed, and therefore Gr.IV of T91 differs from Gr.IV of Grade 92 shown in Figures 12 and 15, though the values of $Q V C$ are similar. If the boundary of Gr.IV could expand to a lower temperature and longer time duration, we can expect that the trend of the breakdown in strength of Gr.IIIa will be moderated. However, a temperature of $600{ }^{\circ} \mathrm{C}$ is the far beyond the realm of Gr.IV and moreover the Z-phase is inevitably formed at $600{ }^{\circ} \mathrm{C}$. Therefore, to investigate the creep behavior of Grade 91, long-term creep tests longer than $100000 \mathrm{~h}$ are desired.

\section{Discussion}

\subsection{Degradation of 100000 h Rupture Strength of 9Cr Martensitic Steel with High Strength}

The rupture strengths of P92/MJP, T92/MJT, and T91/MGC decrease unexpectedly after tens of thousands of hours at a specific temperature between $600-650{ }^{\circ} \mathrm{C}$ as explained in Sections $4.3-4.5$. Figure 21 compares the rupture strength of these steels at $600{ }^{\circ} \mathrm{C}$. The $\sigma-t_{r}$ relationship for a plate of Grade $91 / \mathrm{MgC}$ is also added in Figure 21 referring to a data sheet of No.43A (NIMS, 2014). The Ni content of $\mathrm{MgC}$ heat is $0.04 \%$, which is rather low compared to that of T91/MGC $(0.28 \%)$ shown in Table 3. The $\sigma-t_{r}$ relationships of all data for Grade $91 / \mathrm{MgC}$ and the related results of the regression analyses are shown in Figure A8. Rupture data of $t_{r}>100000 \mathrm{~h}$ for Grade P91 (Panait, 2010b) is also plotted in Figure 21 (symbolized as P91/Panait). The rupture strength decreases along Gr.I $\rightarrow$ Gr.II $\rightarrow$ Gr.III $\rightarrow$ Gr.IIIa (Gr.IIIb) in S-shape for all the steels. In Gr.II, as explained in Sections 4.3.3, 4.4.3, and 4.5.3 these steels are strengthened by the Laves phase. In the Gr.IIIa region of Figure 21, the strengthening effect of MX is partially diminished by the formation of the Z-phase and as a result, the degradation in strength obviously occurs for these steels. The behavior of Gr.III lies between that of Grs.II and IIIa for all the steels. The $100000 \mathrm{~h}$ rupture strength at $\sim 60{ }^{\circ} \mathrm{C}$ can be estimated from rupture data mainly in Gr.IIIa regions and the maximum allowable tensile strength is determined with reference to the 100 $000 \mathrm{~h}$ rupture strength at a specific temperature. Although the test conditions for Gr.IIIa are not defined strictly, Gr.IIIa for many high Cr martensitic steel with high strength are usually observed in a similar temperature and stress range. Rupture strengths for $100000 \mathrm{~h}$ of these steels are estimated from Equation 1 substituting the QVC values obtained for each steel and a specific temperature, and the results are summarized in Table 16. The 
rupture strength of Grade $91 / \mathrm{MgC}$ at $625^{\circ} \mathrm{C}$ is not obtained due to the lack of long-term data as shown in Figure A8. In the calculation when the regression lines around $100000 \mathrm{~h}$ become complicated, the regression line for the appropriate data group is selected by considering the following situation: (i) the rupture strength decreases in the S-shape for all the steels investigated and (ii) there is a tendency for the slope of a $\sigma-\log t_{r}$ relationship to become gentler beyond $100000 \mathrm{~h}$ compared to that of Gr.IIIa.

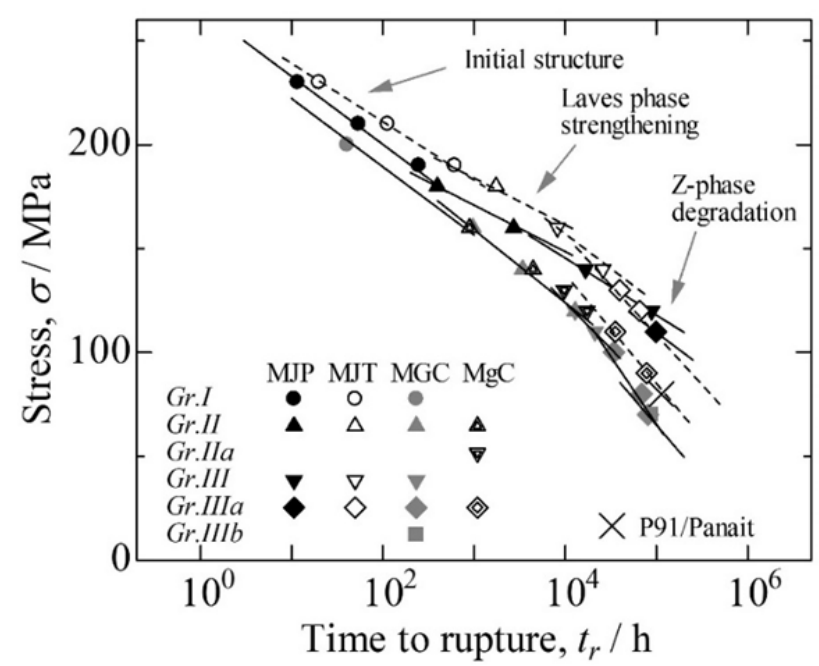

Figure 21. Comparison among the $\sigma-t_{r}$ relationships at $600^{\circ} \mathrm{C}$ of P92/MJP, T91/MGC, and Grade $91 / \mathrm{MgC}$. Rupture data for P91/Panait is presented by Panait et al. (2010b)

Table 16. Estimated rupture strength for $100000 \mathrm{~h}$ at temperatures indicated for P92/MJP, T92/MJT, T91/MGC, and Grade 91/MgC. Symbols in parentheses denote each corresponding data group used for the estimation

\begin{tabular}{|c|c|c|c|c|}
\hline $\mathrm{MPa}$ & MJP & MJT & MGC & $\mathrm{MgC}$ \\
\hline $600^{\circ} \mathrm{C}$ & 109.4 (IIIa) & 108.5 (IIIa) & 65.9 (IIIa) & 85.1 (IIIa) \\
\hline $625^{\circ} \mathrm{C}$ & 84.4 (IIIa) & 78.9 (IIIa) & $46.3(I I I b)$ & - \\
\hline $650^{\circ} \mathrm{C}$ & $59.5(I I I a)$ & $52.6(I V)$ & $43.9(I V)$ & $40.3(I I I b)$ \\
\hline
\end{tabular}

The rupture strength for the steels are MJP $>$ MJT $>\mathrm{MgC}>\mathrm{MGC}$ and those for Grade 92 are naturally higher than those for Grade 91. In most cases shown in Table 16 the $100000 \mathrm{~h}$ strength are calculated using data in Gr.IIIa, where the degradation in rupture strength is a critical issue. Tamura (2015a) showed by analyzing long-term rupture data of Grade 122 (NIMS, 2013) that the estimation method based on an exponential law introduced here is appropriate compared to the methods based on the power law usually used (Kimura, 2009) and also indicated that in any case long-term rupture data is necessary to ensure high accuracy of the estimation for $100000 \mathrm{~h}$ rupture strength. If the longest rupture data of high strength steel is limited to within $10000 \mathrm{~h}$, the effect of the Laves phase strongly reflects the estimated $100000 \mathrm{~h}$ rupture strength which results in over estimation. For the estimation of $100000 \mathrm{~h}$ rupture strength of high strength martensitic steel with high accuracy, rupture data longer than approximately $70000 \mathrm{~h}$ is required, because microstructure changes unexpectedly around the Gr.IIIa region. It should be remembered that the applied load in actual functional power plants is far lower than the $100000 \mathrm{~h}$ rupture strength, and therefore, it is difficult to estimate either the microstructure or the related mechanical behavior or both, although the changes in the microstructure are considered to be moderated compared to those of Gr.IIIa. It is again found from Table 16 and Figure 21 that there are not only certain levels of differences between Grade 91 and Grade 92, but also there are still differences among the heats belonging to the same steel grade. Such differences are also confirmed in the other heats of Grades 91 and 92 than those shown in Table 16 (NIMS, 2007; 2013 ; 1014; 2018). Furthermore, the reasons for these differences have been studied (Kimura et al., 2013; Sawada et al., 2014a; 2014b; 2019a), however they have not been sufficiently clarified. Therefore, though the creep strength of Grade 92 is considered to be credible, there is room for further improvement. 


\subsection{Causes of Degradation}

\subsubsection{A Question}

Many researchers (Kushima et al., 1999; Suzuki et al., 2003; Sawada et al., 2006; Danielsen, 2007; Hald, 2008; Kimura et al., 2013) agree that the main cause of the unexpected degradation in long-term rupture strength is the partial disappearance of the strengthening factors of MX due to the formation of the Z-phase. Sawada et al. (2007) indicated that TTP curves of the Z-phases for Grades 91 and 92 are very similar. Moreover, Kocer, et al. (2009) reported that the $\mathrm{Cr}$ content is the main driving force for the formation of the Z-phase which is secondarily impacted by the $\mathrm{Nb}, \mathrm{V}$, and $\mathrm{N}$ of the alloy system. Therefore, considerations based on the thermodynamic stability and the TTP diagram for the Z-phase in martensitic steel should show few differences in the degree of degradation in rupture strength of Grades 91 and 92 steels caused by the formation of the Z-phase. Namely, the differences in the degradation shown in Figure 21 should be explained by considering the formability of the Z-phase. Sawada et al. (2006) indicated that the number density of the Z-phase $\left(\rho_{Z}\right)$ in a gauge portion is higher than of a grip portion of a ruptured specimen and, therefore that the larger rupture elongation of T91/MGC causes the higher $\rho_{Z}$ which leads to severe degradation in T91/MGC compared to that of T92/MJT. Sawada et al. (2011) also reported using creep-interrupted specimens of T91/MGC tested at $600{ }^{\circ} \mathrm{C}$ under $70 \mathrm{MPa}$ (Gr.IIIa conditions) that the number density of MX decreases with increasing creep strain in response to the increase in the $\rho_{Z}$. However, similar differences in degradation as shown in Figure 21 are confirmed for time to a specific strain regardless of the values of rupture elongation. Figure 22 shows the relationships between the $\sigma-t_{1}$ relationships at $600{ }^{\circ} \mathrm{C}$ for MJP, MJT, and MGC. The values of the start time of the Z-phase formation at $600{ }^{\circ} \mathrm{C}$ for T92/MJT and T91/MGC are $\sim 8000$ and 10000 h, respectively (Sawada et al., 2007) and each time is within each Gr.IIIa region in Figure 22. Therefore, the Z-phase is considered to have already been formed within the time duration of $1 \%$ strain in Gr.IIIa of both steels. The $\rho_{Z}$ s for MGC and MJT can be estimated as follows: The $\rho_{Z}$ of MGC crept to a $1 \%$ strain at $600{ }^{\circ} \mathrm{C}(70$ $\mathrm{MPa}, 52581 \mathrm{~h}$ ) can be read as $2 \mathrm{E} 11 \mathrm{~m}^{-2}$ from the previous work (Sawada et al., 2011) and on the other hand, although the $\rho_{Z}$ of creep-interrupted specimens of MJT is not yet investigated, the $\rho_{Z}$ value of MJT crept to $1 \%$ strain at $600{ }^{\circ} \mathrm{C}(100 \mathrm{MPa}, 73000 \mathrm{~h})$ is estimated to be a maximum of $1.4 \mathrm{E} 11 \mathrm{~m}^{-2}$ from the $\rho_{Z}$ data in ruptured specimens (Sawada et al., 2006). Even under the same specific strain condition of $1 \%$, the $\rho_{Z}$ of T91/MGC is higher than that of T92/MJT, which should also be correlated to the degradation in strength shown in Figure 22. Therefore, if the reasons for the degradation in strength for $t_{r}$ and $t_{1}$ in each Gr.IIIa shown in Figures 21 and 22 are the same, considering the variations of $\rho_{Z}$ mentioned above it is insufficient to consider that the larger rupture elongation is responsible for the formation of a larger amount of the Z-phase, which leads to lower rupture strength of MGC. Therefore, a new model is needed to explain the degradation in long term rupture strength of martensitic steel.

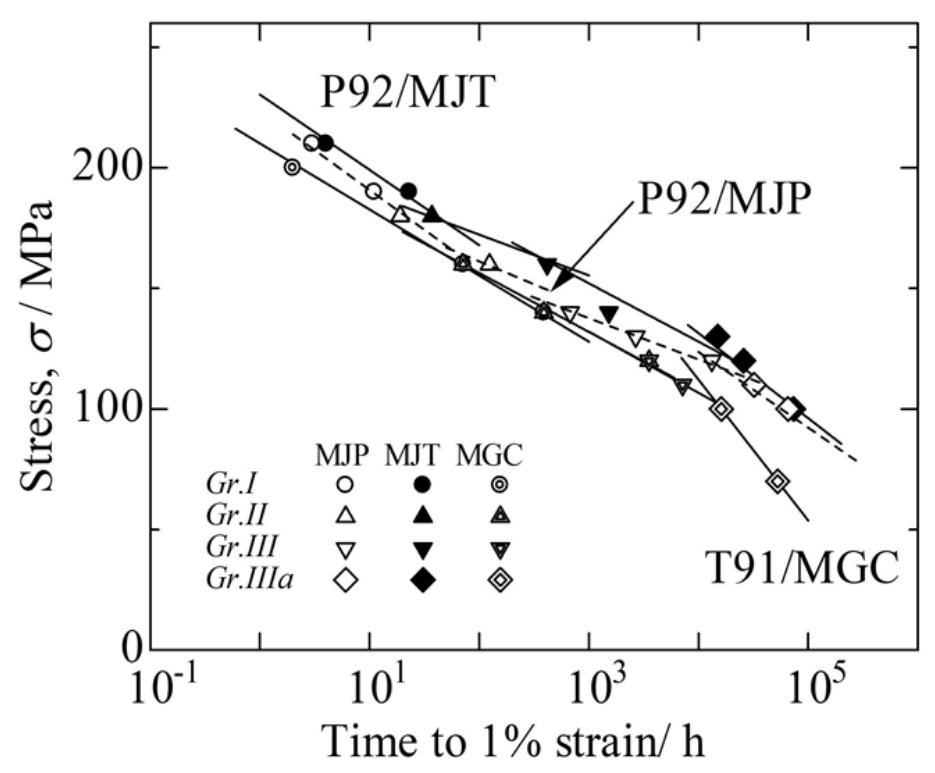

Figure 22. Comparison between the $\sigma-t_{1}$ relationships at $600{ }^{\circ} \mathrm{C}$ of P92/MJP, T92/MJT, and T91/MGC 


\subsubsection{Modeling of Microstructural Variations during Creep of Gr.IIIa}

Table 17 shows the $t_{r}$ at $600{ }^{\circ} \mathrm{C}$ under a stress indicated in the Gr.IIIa region, $\rho_{Z}$, and the $E L$ for each steel. For the data of P91/Panait (Panait et al., 2010b) the $\rho_{Z}$ is not yet investigated, but the $\rho_{Z}$ is reported as a few compared to that of $\mathrm{MX}$, and the $E L$ is estimated from the creep curves shown in their paper. Table 17 certainly shows a tendency that $\rho_{Z}$ is higher and thus $t_{r}$ is shorter for steels with higher $E L$ values. However, when we compare the data with $t_{r}=30000-40000 \mathrm{~h}$ among MJP, MJT, and MGC, it is found from Table 17 that this

Table 17. Comparison of the time to rupture at $600{ }^{\circ} \mathrm{C}$ under the stress indicated, the number density of Z-phase in a ruptured specimen $\left(\rho_{Z}\right)$, Rupture elongation $(E L)$, and the activation volume for Gr.IIIa among the steels indicated. Creep data of Grade P91 is presented by Panait et al. (2010b)

\begin{tabular}{|c|c|c|c|c|c|}
\hline Steel & Stress / MPa & $t_{r} / \mathrm{h}$ & $\rho_{z} / 1 \mathrm{E} 11 / \mathrm{m}^{-2}$ & $E L(\%)$ & $V / \mathrm{cm}^{3} \mathrm{~mol}^{-1}$ \\
\hline P92/MJP & 130 & 38067 & & 20 & 505.1 \\
\hline T92/MJT & 130 & 39539 & 1.5 & 11 & 348.5 \\
\hline \multirow{2}{*}{ T91/MGC } & 100 & 34141 & 3.4 & 22 & \multirow{2}{*}{256.4} \\
\hline & 70 & 80737 & 7.6 & 33 & \\
\hline Grade $91 / \mathrm{MgC}$ & 90 & 78237 & 1.9 & 28 & 318.2 \\
\hline Grade P91/Panait & 80 & 113431 & (a few) & 7.3 & \\
\hline
\end{tabular}

Table 18. Average temperature, time to rupture, $Q V C_{\max }$, and $M C R$ for each data group of P92/MJP, T92/MJT, and T91/MGC. The minimum creep time for detectable degradation as compared with an adjacent shorter time data group (MTD) and the start time for Z-phase formation $(T Z F)$ are also shown. Strains corresponding to each time are shown in $\%$ in each parenthesis

\begin{tabular}{|c|c|c|c|c|}
\hline \multirow{2}{*}{ Steel } & \multirow{2}{*}{ Items } & \multicolumn{3}{|c|}{ Time $/ \mathrm{h}$ (strain, \%) } \\
\hline & & Gr.II & Gr.III & Gr.IIIa \\
\hline \multirow{6}{*}{ P92/MJP } & Av. Temp. $/{ }^{\circ} \mathrm{C}$ & 640 & 634 & 634 \\
\hline & Av. $t_{r} / \mathrm{h}$ & 2974 & 18618 & 62811 \\
\hline & $Q V C_{\max }$ & $1015(2)$ & $1857(1)$ & $4968\left(0.5^{* *}\right)$ \\
\hline & $M T D$ & - & $9453(2)$ & $27074(1)$ \\
\hline & $M C R$ & $2658(5)$ & $9453(2)$ & $27074(1)$ \\
\hline & $T Z F$ & 3500 & 3700 & 3700 \\
\hline \multirow{6}{*}{ T92/MJT } & Av. Temp. $/{ }^{\circ} \mathrm{C}$ & 637 & 613 & 622 \\
\hline & Av. $t_{r} / \mathrm{h}$ & 5136 & 25317 & 49878 \\
\hline & $Q V C_{\max }$ & $174(1)$ & $220\left(0.5^{* *}\right)$ & $4815\left(0.5^{* *}\right)$ \\
\hline & $M T D$ & - & $1990(1)$ & $27220(1)$ \\
\hline & $M C R$ & $4608(5)$ & $14733(2)$ & $27220(1)$ \\
\hline & $T Z F$ & 3600 & 4700 & 4300 \\
\hline \multirow{6}{*}{ T91/MGC } & Av. Temp. $/{ }^{\circ} \mathrm{C}$ & 610 & 580 & 583 \\
\hline & Av. $t_{r} / \mathrm{h}$ & 3709 & 24613 & 58699 \\
\hline & $Q V C_{\max }$ & $830(1)$ & $7288\left(1^{*}\right)$ & $1260\left(0.2^{* *}\right)$ \\
\hline & $M T D$ & - & $17420(2)$ & $12377\left(0.5^{* *}\right)$ \\
\hline & $M C R$ & $2197(2)$ & $17420(2)$ & $29527(1)$ \\
\hline & $T Z F$ & 6200 & 13500 & 13500 \\
\hline
\end{tabular}

Note. Bold-italics: shorter time than TZF

* Strain for Vmax is $0.5 \%$ and time to $0.5 \%$ strain is $1614 \mathrm{~h}$.

** No available data below the indicated strain. 
tendency does not always hold true for the case of P92/MJP. The $t_{r}$ of MJP is longer than that for MGC, though the ELs for both steels are comparable. Instead, the corresponding values of $V$ for Gr.IIIa listed in Table 17 seem to show the better correlation among $V, \rho_{Z}$, and $t_{r}$. Certainly, though the correlation between $V$ and $t_{r}$ under $130 \mathrm{MPa}$ is not so good for MJP and MJT, the tendency of correlation between $V$ and $t_{r}$ may be true under lower stresses. Figure 21 seems to indicate that the $t_{r}$ of MJP can be longer than that of MJT under a lower stress corresponding to beyond hundreds of thousands of hours. The slope of the $\sigma-\log t_{r}$ relationship denotes that the rate of degradation in strength is inversely proportional to $V$ according to Equation 10. Namely, the lower $V$ may evoke the higher $\rho_{Z}$ in the Gr.IIIa region of the high $\mathrm{Cr}$ martensitic steel containing MX. Therefore, it can be naturally understood that $V$ is considered to be correlated with $\rho_{Z}$ and $t_{r}$ as seen in Table 17. Moreover, a similar correlation should hold even for a specific strain of $1 \%$ as estimated from Figure 22 . Furthermore, such a decrease in $V$ should be correlated with an increasing degree of HRHD as explained in Sections $4.1-4.5$, and thus with a decrease in $t_{r}$.

In Sections 4.3 - 4.5, the variations in $Q V C$ during creep of P92/MJP, T92/MJT, and T91/MGC were explained for each data group based on the metallurgical observations. Degradation in strength for Grs.IIIa of P92/MJP and T91/MJT, and Grs.IIIa and IIIb of T91/MGC is a critical issue. In these data groups the formation of the Z-phase is common at $600{ }^{\circ} \mathrm{C}$ and therefore, we should be able to construct a metallurgical model of the microstructural variations for the degradation in strength through the creep behavior of these three steels. The formation of the Z-phase is also confirmed for Grs.II and $I I I$ at $600{ }^{\circ} \mathrm{C}$. Therefore, the average temperature, the average $t_{r}$, the time to the $Q V C_{\text {max }}, M T D, M C R$, and TZF for Grs.II, III, and IIIa of MJP, MJT, and MGC, respectively, are surveyed and the results are shown in Table 18, where MTD denotes the minimum creep time for the detectable degradation at $600{ }^{\circ} \mathrm{C}$ when compared to an adjacent shorter time data group, which was determined using both the average $t_{\varepsilon, r}-\sigma$ and $t-\varepsilon$ diagrams for each data group, and TZF is the start time for the Z-phase formation estimated from Sawada's work (Sawada et al., 2007). In Table 18, the corresponding creep strains to each time are also shown in parentheses.

Combining the information obtained in Sections $4.3-4.5$ and Table 18, the microstructural degradation processes during creep in Gr.IIIa of high Cr martensitic steel with high strength are considered to be as follows: From the beginning of transient creep, SBSD and grain boundary strengthening by $\mathrm{M}_{23} \mathrm{C}_{6}$ and the Laves phase occurs and the $Q V C$ reach maximum for each. Subsequently, the $Q V C$ begin to decrease and simultaneously HRHD begins in the vicinity of the weakest boundaries. At that time, $\mathrm{M}_{23} \mathrm{C}_{6}$ and the Laves phase on the boundaries are quickly coarsened and thus the $Q$ decreases, because diffusion of atoms becomes active according to an increase in creep strain in an HRHD zone.

Additionally, in a heavily deformed HRHD zone, some of the strengthening particles of MX should be dissolved into the matrix and be transported towards the nearby boundaries by gliding dislocations. When the dislocations reach the vicinity of the boundaries, the more stable compound of the Z-phase rather than MX is newly formed from the super-saturated matrix with the constituent elements of $\mathrm{MX}$, i.e., $\mathrm{Nb}$ and $\mathrm{V}$, along with an abundant supply of $\mathrm{Cr}$ from near the boundaries, or the excess $\mathrm{Nb}$ and $\mathrm{V}$ atoms are used to coarsen the pre-existing Z-phase, hereby the $Q$ decreases further. As result of these sequential reactions, the creep strength at a strain in transient creep of Gr.IIIa becomes lower compared to that of Gr.III. The detailed mechanisms for the promotion, acceleration or both of the diffusion and dissolution of MX, as well as the transportation towards the boundaries of $\mathrm{Nb}$ and $\mathrm{V}$ are discussed in the later section of 5.2.6. Even when $Q$ continues to decrease in this manner with increasing strain transient creep holds if $\Delta\left[V_{s}\right]+\Delta C_{s}<\Delta\left[Q_{s}\right]<0$ is satisfied, as shown in Tables 11 and 14 . When $Q$ decreases further due to the increase in the formation of the Z-phase accompanying the consumption of some MX particles, and the decrease in $\left[Q_{s}\right]$ is equal to the decrease in $\left[V_{s}\right]+C_{s}$, the creep rate reaches an $M C R$ point and then, accelerating creep begins.

In accelerating creep, an increase in the sub-grain size, the coarsening of the Z-phase consuming some MX particles, and the coarsening of both the Laves phase and $\mathrm{M}_{23} \mathrm{C}_{6}$ are progressing and eventually time to rupture is shortened in Gr.IIIa unexpectedly. These degradation processes are illustrated schematically in Figure 23. Two points are significant in the illustration: First, SBSD followed by HRHD processes are the essence of transient creep of high $\mathrm{Cr}$ martensitic steel with high strength and second, degradation in strength begins to occur even in the transient creep region, because the Z-phase begins to form dissolving some MX particles with the aid of straining within a heavily deformed HRHD zone. This model denotes that a chronological sequence for the events in Gr.IIIa is roughly expressed as

$$
\text { SBSD, DFRP of } \mathrm{M}_{23} \mathrm{C}_{6} \text {, Laves phase } \leq Q V C_{\text {max }} \leq \mathrm{HRHD} \leq T Z F \leq M T D \leq M C R<t_{r}
$$


This model is able to clearly explain the mechanism for the degradation in strength. The values of time indicated in Table 18 for Gr.IIIa are roughly satisfied Equation 12. However, there are still some questions about this model concerning the existence of $Q_{\max }$, the formation of the Z-phase at a small nominal strain no larger than an $M C R$ point as much as degrading the strength, and the existence of HRHD. These problems shall be discussed in the subsequent sections.

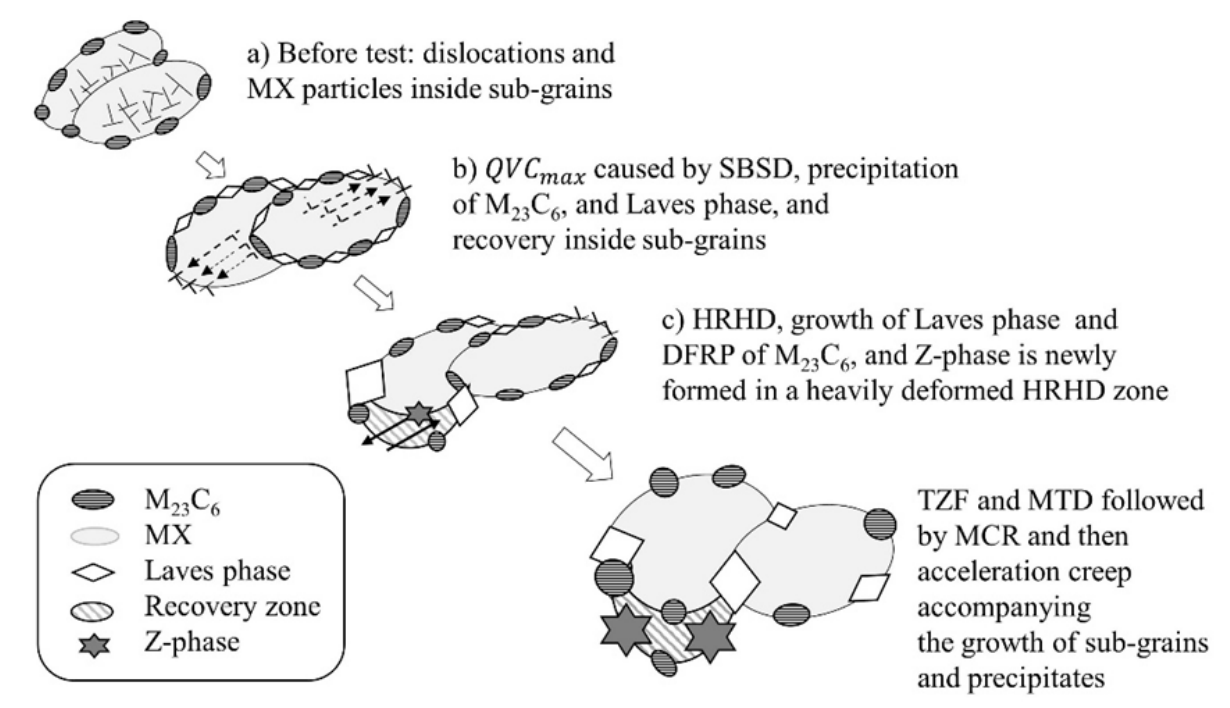

Figure 23. Schematic illustration for microstructural degradation during creep for Gr.IIIa of high Cr martensitic steel with high strength

\subsubsection{Existence of $Q_{\max }$ in Gr.IIIa}

The $Q_{\max } \mathrm{s}$ are not clearly confirmed in Gr.IIIa for all steels investigated as shown in Table 18 . This problem can be explained as follows: Figure 24 compares the relationships between $\varepsilon$ and $Q$ for Grs.III and IIIa (IIIb) of MJP, MJT, and MGC. As shown in Figure 24, the hardening process due to SBSD, $\mathrm{M}_{23} \mathrm{C}_{6}$, and Laves phase is not confirmed for Gr.IIIa like a process b) in Figure 23. However, we can believe the existence of the hardening process at a very early stage of transient creep in Gr.IIIa. The first reason is that the values of $Q_{\max }$ for each Gr.IIIa are as large as those for not only Gr.III of all the steels discussed but also Gr.III of 9Cr-4W steel, where the hardening caused by dislocations, $\mathrm{M}_{23} \mathrm{C}_{6}$, and Laves phase are confirmed as shown in Figures 9, 14, 17, and 20. The second reason is that we can infer the existence of peaks in $Q$ for Gr.IIIa of all the steels discussed by analogy: In the adjacent data group of $G r . I I I$ we can easily believe the existence of a peak in $Q$ for MJT maybe at a small strain below $0.5 \%$ like those for MJP and MGC, because the creep behavior for all the steels discussed is similar with each other as explained in Sections in $4.3-4.5$ except that the peaks in QVC are lacking for MJT and moreover, the degradation behavior during creep for Gr.IIIa of all the steels discussed are similar to the behavior of Gr.III to some extent, though the SBSD is weaken in Gr.IIIa due to higher recovery rate under low stresses. Moreover, the follows are other evidences for the real existence of the boundary hardening process in an early stage of transient creep for Gr.IIIa. As shown in Table 18 the $Q_{\max }$ for Gr.IIIa of MGC occurs at $\varepsilon=0.2 \%$ for $1260 \mathrm{~h}$ in average. However, it can be evaluated by analyzing creep data of T91/MGC tested at $600{ }^{\circ} \mathrm{C}$ and $70 \mathrm{MPa}$ (Sawada et al., 2011), which is a member of Gr.IIIa in this study, that a creep strain of $0.2 \%$ at $600{ }^{\circ} \mathrm{C}$ and $70 \mathrm{MPa}$ for $Q_{\max }$ is corresponding to creep time of approximately $4000 \mathrm{~h}$, as shown in Figure A7. At this time, the dislocation density inside sub-grains of a crept specimen at $600{ }^{\circ} \mathrm{C}$ and $70 \mathrm{MPa}$ tends to decrease, but the hardness does not change as compared with that of before the test (Sawada et al., 2011). Therefore, grain boundary hardening, i.e., SBSD, $\mathrm{M}_{23} \mathrm{C}_{6}$, and the Laves phase should possibly occur, because Laves phase is very fine, approximately $100 \mathrm{~nm}$ at $600{ }^{\circ} \mathrm{C}$ for $1000 \mathrm{~h}$ near the condition of which the $Q$ shows the maximum for $\mathrm{Gr}$.IIIa of MGC (Suzuki et al., 2000). An above-mentioned combination of $\varepsilon=0.2 \%, 600^{\circ} \mathrm{C}, 70 \mathrm{MPa}$, and $4000 \mathrm{~h}$ for MGC is estimated from the published data of a $t-\dot{\varepsilon}$ diagram, as explained in Sections 4.5.1 and 4.5.6. When we estimate the corresponding time using a $t-\varepsilon$ diagram, an approximately $1000 \mathrm{~h}$ is obtained for $t_{0.2}$ instead 
referring to the data $t_{0.2}=261 \mathrm{~h}$ under $100 \mathrm{MPa}$ as shown in Figure A7. In this case, the grain boundary hardening with recovery of inside sub-grains is also confirmed. Therefore, in any case, the estimated microstructure for the $t_{0.2}$ of a crept specimen of MGC at $600{ }^{\circ} \mathrm{C}$ under $70 \mathrm{MPa}$ possibly supports the existence of $Q_{\max }$ at a very early stage of transient creep for Gr.IIIa.

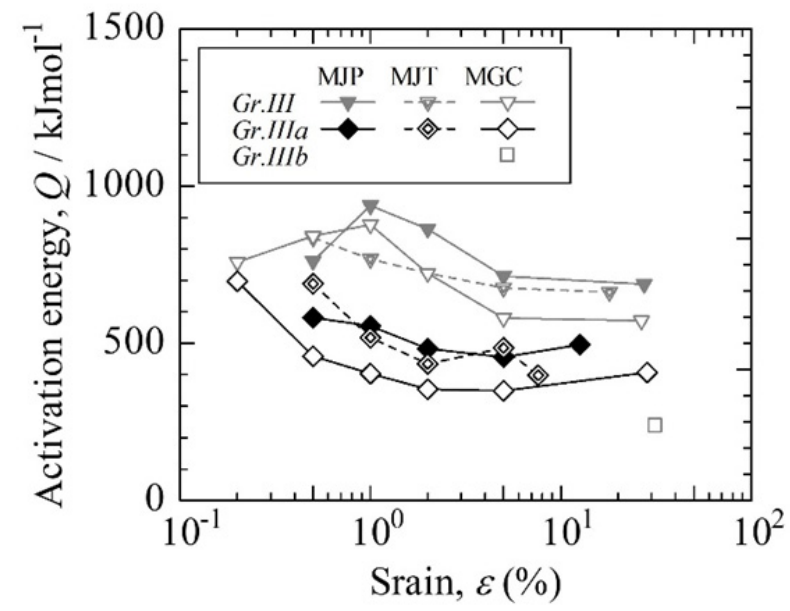

Figure 24. Comparison among the activation energy during creep for Grs.III, IIIa, and IIIb of MJP, MJT, and MGC

\subsubsection{Formation of an HRHD Zone}

A concept of HRHD is evoked from the following two points of views: The first one is that the three parameters of $Q V C$ decrease after $Q V C_{\max }$ simultaneously in a later section of transient creep as seen in Figures 4, 9, 14, 17 , and 20, which imagines that recovery should take place within a small nominal strain range after remarkable strain hardening by SBSD, DFRP of $\mathrm{M}_{23} \mathrm{C}_{6}$, and precipitation of fine Laves phase at the beginning of creep. The second one is that the $Q V C$ for the welded joints are small as compared to those of the base metal as shown in Figure 1, Tables 1, and 2. Hence, it follows that we have explained the creep phenomena of the martensitic steel on the assumption that HRHD takes place by analogy when $V$ decreases as explained in Sections 4.1 to 4.5. Namely, if we can assume the existence of a locally recovered zone, we can easily suppose the strain concentration in this area and therefore, we can judge that the formation of Z-phase is promoted in an HRHD zone based on the experimental results that creep strain promotes the formation of Z-phase (Sawada et al., 2006; 2011). Therefore, the accelerating formation of Z-phase in an HRHD zone is believed even within a small nominal strain not larger than a strain at an $M C R$ point. The formation of Z-phase near an HRHD zone progresses accompanying the dissolution or disappearance of a strengthening factor of MX, which results in the degradation in strength even in a small strain of $1 \%$ as shown in Figure 22. There is no contradiction in the above explanation of the creep phenomena of martensitic steel and therefore, we come to believe the existence of HRHD. We already confirmed that $V$ decreases for the welded joint as shown in Figure 1 and so that, small values of $V$ observed in transient creep of martensitic steel evoke us the occurrence of local or heterogeneous deformation like a welded joint and the decrease in rupture strength. Sawada, Bauer, Kauffmann, Mayr, and Klenk (2010) reported that the $\rho_{Z}$ 's in HAZ of the ruptured specimens at $600{ }^{\circ} \mathrm{C}$ of the welded joints of Grade E911 are approximately one order of magnitude larger than those for the base metal, which depend on the time to rupture and the location of sampling in HAZ. The $\rho_{Z}$ of base metal is approximately $1.7 \mathrm{E} 10 \mathrm{~m}^{-2}$ and is similar with that of T92/MJT (Sawada et al., 2006). The increases in $\rho_{Z}$ for the welded joints as compared with the base metal were explained due to the effects of the increased precipitation site for Z-phase and the multi-axial stress in HAZ. They showed rupture data only at $600{ }^{\circ} \mathrm{C}$ and therefore, though the values for $Q$ and $C$ cannot be calculated, the values of $V$ calculated from their data using Equation 10 are $298 \mathrm{~cm}^{3} \mathrm{~mol}^{-1}$ for up to 10000 $\mathrm{h}$ and $73 \mathrm{~cm}^{3} \mathrm{~mol}^{-1}$ for $10000-35000 \mathrm{~h}$, respectively. These values of $V$ are extremely lower than those for Grade 92 shown in Figures 14 and 20 and the $t_{r}$ 's are much shorter than those for T92/MGC. These data certainly show that local deformation of the welded joint causes a decrease in $V$, an increase in $\rho_{Z}$, and thus a decrease in $t_{r}$, and certainly these data also satisfies a sufficient condition for the existence of HRHD during creep of high $\mathrm{Cr}$ martensitic steel as explained in Sections 4.3-4.4. 
Experimentally, a locally recovered zone is certainly observed in a ruptured specimen for T91/MGC (Kushima et al.,1999) and T92/MJT (Sawada \& Kimura, 2019b) and also in the crept specimens for accelerating creep of MGC including an MCR point (Kimura et al., 2002). However, a locally recovered area in a crept specimen to a small strain in transient creep has not yet been confirmed. Moreover, not only the size, shape, and distribution, but also the degree of straining of a locally recovered zone, i.e., the characteristics of an HRHD zone, are not yet investigated systematically. These factors may influence the formability of Z-phase near HRHD zones, and thus the degradation in strength. When such information on an HRHD zone is clarified, the variations in the degradation in strength for Gr.IIIa as shown in Figure 21 can be explained more deeply. Morooka, Tomoda, Adachi, Morito, and Kamiyama (2008) observed heterogeneous deformation inside a sub-block of martensitic structure employing both an electron back-scattered diffraction method and a neutron diffraction method, and therefore, the characterization of an HRHD zone during creep shall be clarified more deeply in future.

\subsubsection{Formation of Z-Phase under Low Stresses}

Danielsen (2007) and Danielson and Hald (2009) proposed a new model for the formation of Z-phase particles by $\mathrm{Cr}$ diffusion onto an MX particle from the high $\mathrm{Cr}$ martensite matrix. Cipolla et al. (2010) supported the Danielsen's model and gave the experimental evidence using a model alloy of carbon-free Fe-12Cr-1.3Ni-NbVN alloy aged at $600-700{ }^{\circ} \mathrm{C}$ for maximum $10000 \mathrm{~h}$. However, it is found from simple calculations on T91/MGC that the diffusion distance of $\mathrm{Cr}$ in alpha-iron at $600{ }^{\circ} \mathrm{C}$ is more than one order of magnitude larger as compared with the estimated inter-particle distance of MX: Sawada et al. (2011) reported that the number density of MX $\left(\rho_{M X}\right)$ of as received T91/MGC is approximately $8 \mathrm{E} 12 \mathrm{~m}^{-2}$ and, therefore the inter-particle distance of MX is estimated to be $\sqrt{1 / \rho_{M X}}=0.35 \mu \mathrm{m}$. On the other hand, the diffusion distance of $\mathrm{Cr}$ in alpha-iron is $4.1 \mu \mathrm{m}$ at $600{ }^{\circ} \mathrm{C}$ for $1000 \mathrm{~h}$. Here, the calculation of the diffusion distance for $\mathrm{Cr}$ atom, i.e., $2 \sqrt{D t}$, is performed assuming $D_{0}=0.00023 \mathrm{~m}^{2} \mathrm{~s}^{-1}, Q=238.8 \mathrm{kJmol}^{-1}$ (Oikawa, 1986), $t=1000 * 3600 \mathrm{~s}$, and $T=873 \mathrm{~K}$ in the relation $D=D_{0} \exp (-Q / R T)$. Therefore, we can understand the diffusion distance of $\mathrm{Cr}$ is much larger than the inter-particle distance of MX during creep of T91/MGC even for the conditions of $600{ }^{\circ} \mathrm{C}$ for $1000 \mathrm{~h}$. The inter-particle distance of MX increases with not only increasing creep time and temperature, but also the amount of Z-phase formed, however the diffusion distance of $\mathrm{Cr}$ in alpha-iron also increases with increasing time and temperature. The inter-particle distance of MX in a ruptured specimen of T91/MGC at $600{ }^{\circ} \mathrm{C}$ and $70 \mathrm{MPa}$ $\left(t_{r}=80737 \mathrm{~h}\right)$ is estimated as $\sqrt{1 / \rho_{M X}}=1.41 \mu \mathrm{m}$ (Sawada et al., 2011) and the corresponding diffusion distance becomes $37.1 \mu \mathrm{m}$. Namely, except below $10 \mathrm{~h}$ at $600{ }^{\circ} \mathrm{C}$, the diffusion distance of $\mathrm{Cr}$ in alpha-iron is always larger than the inter-particle distance of MX at $600^{\circ} \mathrm{C}$. Therefore, according to Danielsen's model (2007), fine particles that are similar in composition to the $\mathrm{Z}$-phase, $(\mathrm{Cr}, \mathrm{Nb}, \mathrm{V}) \mathrm{X}$, must be easily formed within a very short time duration, e.g., several tens of hours, because the Z-phase is thermodynamically more stable than MX (Kocer et al., 2009). However, such particles are not observed until at least $\sim 10000 \mathrm{~h}$ at $600{ }^{\circ} \mathrm{C}$ (Sawada et al., 2007). Based on the classical nucleation and growth theory of precipitation (Turnbull \& Fisher; 1949), the interfacial energy of a Z-phase particle with a tetragonal structure in the martensitic steel matrix is considered to be substantially larger than that of the MX phase with a cubic structure. Therefore, numerous $\mathrm{Nb}$ and $\mathrm{V}$ atoms are expected to be transported to the large-angle boundaries with the aid of moving the dislocations, PAGBs, and the Z-phase where the tetragonal structure is formed. This mechanism will be explained in Section 5.2.6. An alternative interpretation is possible: Even if the fine particles of $(\mathrm{Cr}, \mathrm{Nb}, \mathrm{V}) \mathrm{X}$ could be formed within a short time duration based on Danielsen's model, provided that their structure is cubic and their interfacial energy is low, these structures are unstable due to an interaction with the dislocations (Tamura, Iida, Kusuyama, Shinozuka, \& Esaka, 2004), which will also be explained in Section 5.2.6. Therefore, the number density of the $(\mathrm{Cr}, \mathrm{Nb}, \mathrm{V}) \mathrm{X}$ particles will remain extremely low. Also, when the size is below $20 \mathrm{~nm}$, these fine particles were not detected by the elemental mapping method as Z-phase particles (Sawada et al., 2007). Sawada, Kubo, and Abe (2003) demonstrated that when transmission electron microscopy was used, the number density of MX particles $<20 \mathrm{~nm}$ decreases to a very low level after aging for $727 \mathrm{~h}$ at $700{ }^{\circ} \mathrm{C}$ when compared with that of the as-received specimen. Therefore, even if the $(\mathrm{Cr}, \mathrm{Nb}, \mathrm{V}) \mathrm{X}$ particles could be formed, the particles should rapidly grow. In this case, it is difficult to explain why the creep rate continues to decrease consuming the MX strengthening particles during a transient creep range. Although Danielsen's model is simple and straightforward, it is not easily accomplished in high-strength $9 \mathrm{Cr}$ martensitic steel.

Sawada et al. (2011) demonstrated that the number density of MX particles in T91/MGC decreases during creep at $600{ }^{\circ} \mathrm{C}$ and reaches $<10 \%$ of the initial the initial value at rupture $\left(t_{r}=80737 \mathrm{~h}\right)$. Furthermore, the massive Z-phase is formed mainly in the vicinity of the boundaries consuming nearby MX particles. The $\rho_{Z}$ increases with an increase in strain up to a comparable value to that of the MX at rupture. This is clear evidence that the formation of the Z-phase is the main reason for the degradation in the long-term creep strength. By combining 
this data with the $\rho_{Z}$ values for the grip portions (Sawada et al.,2014b) and utilizing the relevant creep curves (Sawada et al., 2011), we can obtain the relationship between $\rho_{Z}$ and $\varepsilon$. The results are shown in Figure 25 . From the figure, $\rho_{Z}$ increases steeply with an increase in strain for $\varepsilon \leq 0.5 \%$. The $\rho_{Z}$ in the gauge portion is definitely larger than that in the grip portion, even though the differences are not so large. On the other hand, the $\rho_{Z}$ in a gauge portion increases gradually with an increase in the logarithm of the creep strain for $\varepsilon \geq 1 \%$ and is approximately three times larger than that of the grip portion. In other words, the majority of the Z-phase was not formed thermally, but formed readily with the aid of the creep strain. The observed creep range, $t \geq$ $10000 \mathrm{~h}$ and $\varepsilon \geq 0.26 \%$, corresponds to accelerating creep because $M C R$ is estimated to occur at $6585 \mathrm{~h}$ and $\varepsilon=0.23 \%$ from the creep curves reported in the literature (Sawada et al., 2011). The rate of increase in $\rho_{Z}$ decreases during accelerating creep (when $\varepsilon \geq 1 \%$ ), which may be due to the coarsening of the Z-phase. There is no data confirming the Z-phase formation in transient creep of MGC at $600{ }^{\circ} \mathrm{C}$ under $70 \mathrm{MPa}$. Instead, the $\mathrm{Z}$-phase formation is estimated to begin approximately near the $M C R$ point, i.e., $6585 \mathrm{~h}$. This estimation does not contradict the observation that the Z-phase begins to form in rupture specimens between $t_{r}=3415(140 \mathrm{MPa})$ and $t_{r}=12859 \mathrm{~h}(120 \mathrm{MPa})$ (NIMS, 2007; Sawada et al., 2007). Therefore, Equation 12 must be interpreted as $S B S D \leq Q_{\max } \leq H R H D \leq T Z F \approx M C R<t_{r}$ for $\mathrm{MGC}$ at $600{ }^{\circ} \mathrm{C}$ under $70 \mathrm{MPa}$, although the existence of both $Q_{\max }$ and HRHD are not yet experimentally confirmed. Under stresses considerably lower than $70 \mathrm{MPa}$, $t_{0.2}$ becomes longer than $10000 \mathrm{~h}$ (Figure A7). Thus, the Z-phase is formed within the time $t_{0.2}$ even though we cannot confirm whether the $t_{0.2}$ belongs to the transient creep. The occurrence of the HRHD is expected to promote the formation of the Z-phase. However, since the sub-grains grow at the same time, the HRHD effect, i.e., the effect of strain concentration, on the formability of the Z-phase will be mitigated.

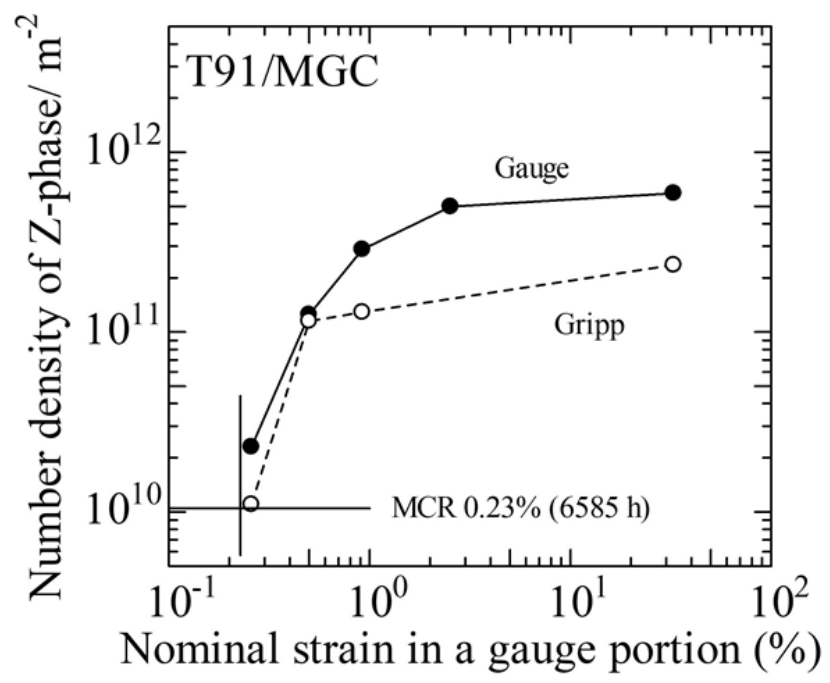

Figure 25. Number density of Z-phase as functions of creep strain for a gauge and grip portion of T91/MGC tested at $600{ }^{\circ} \mathrm{C}$ and $70 \mathrm{MPa}$

We have not yet observed any definite evidence for the formation of the Z-phase in the transient creep after the peak of QVC for Gr.IIIa of MGC along with Grs.III and IIIa of Grades 91 and 92 steel. However, if we assume the existence of an HRHD zone, it can be reasonably believed that the concentrated strain generated in an HRHD zone increases the transportation velocity and the volume of solute atoms, thus promoting the dissolution of MX and the local formation of the Z-phase. The average creep behavior for Grs.III and IIIa of MJP, MJT, and MGC under this scenario is shown in Table 18.

Even though we reasonably deduce the existence of the local Z-phase formation in an HRHD zone during transient creep, the distribution of the Z-phase may not necessarily be heterogeneous. That does not mean the observed values of the $\rho_{Z}$ shown in Figure 25 may not be accurate. Our deduction does not contradict the experimental results made by Sawada (2007) and the formation of the Z-phase in transient creep is possible. The size of an HRHD zone formed in the transient creep may be smaller than the size of the primary austenite grains from the following microstructural point of views: The inter particle distance of the Z-phase is estimated from Figure 25 as 
$2-3 \mu \mathrm{m}(0.5-1 \%$ strain, $30000-50000 \mathrm{~h})$, which corresponds to the situation that several particles $\sim 100 \mathrm{~nm}$ in diameter of the Z-phase (Suzuki et al., 2003) are on the PAGBs for each primary austenite grain $\sim 10 \mu \mathrm{m}$ in diameter (NIMS, 2014). Such situations are frequently confirmed in high-Cr martensitic steel (Sawada et al., 2007). Moreover, the size of a heterogeneously recovered zone observed in the vicinity of the PAGBs (Kushima et al.,1999; Sawada \& Kimura, 2019b; Kimura et al., 2002) is extremely small, several micrometers at most.

\subsubsection{Dislocation Assisted Acceleration of Precipitation Phenomena}

Sawada et al. $(2006 ; 2014 \mathrm{~b})$ reported that the $\rho_{Z}$ in the gauge portion at the rupture of T91/MGC is larger than that in a grip. Moreover, the $\rho_{Z}$ in the gauge portion of T91/MGC is higher than that of T92/MJT with a shorter rupture elongation. This accelerated formation of the Z-phase in T91/MGC is interpreted to be caused by a creep strain (Sawada et al., 2006; 2014b). However, Table 17 shows that an increase in $\rho_{Z}$ is not satisfactorily explained by a nominal strain. Moreover, the mechanism of and reason for the creep strain in the reactions involved in the formation of the Z-phase have not been successfully elucidated. Z-phase particles are generally massive and are typically formed on the boundaries in high-Cr martensitic steel with high strength (Suzuki et al., 2000; Kimura et al., 2002; Sawada et al., 2006). Models for the coarsening of grain boundary precipitates are constructed by combining lattice diffusion and boundary diffusion (Hori \& Saji, 1981; Hassan \& Corney, 2017). That is, solute atoms are transported onto the boundaries by lattice diffusion and then migrate to the targeting precipitates by grain boundary diffusion. The coarsening of Z-phase particles on the boundaries can be understood based on this model because boundary diffusion is faster than lattice diffusion (Shewmon, 1963). Thus, boundary precipitates grow faster than those inside grains (Brailsford \& Aaron, 1969). According to this model, the total amount of precipitates on the boundaries should be ultimately controlled by the total amount of constituent elements transported from inside of grains because the diffusion velocity inside grains is slower than that along the boundaries at average temperatures at which the creep tests are usually conducted. This suggests that the total amount, rather than the size, of precipitates on the boundaries cannot be accelerated when compared to that of the precipitates inside the grains. However, the aforementioned consideration is not correct. The number density of MX particles during the creep of T91/MGC decreases with increasing creep time (Sawada et al., 2011) and the amount of MX disappeared is equivalent to that consumed to form the Z-phase. Moreover, the number density of MX particles during creep in the gauge portion is smaller than that in a grip (Sawada et al., 2014b). Thus, the amount of Z-phase should increase with increasing creep time, considering that there are no significant changes in the size of the MX particles (Suzuki et al., 2000; 2003). Therefore, in addition to the lattice diffusion inside the grains, additional transport systems for the constituent elements of the Z-phase should operate during creep. Numerous dislocations inside the sub-grains in martensitic steel and dislocation pipe diffusion (Shewmon, 1963) may assist in the transportation of the solute atoms. However, there are many excess dislocations in the sub-grains of martensitic steel with high strength, even in the grip portion after long-term testing (Panait et al., 2010b; Fedoseeva, Dudova, \& Kaibyshev, 2016). The existence of excess dislocations or the role of pipe diffusion is not essential for explaining the accelerated formation and coarsening of Z-phase particles in the gauge portion of high-strength martensitic steels. Instead, straining itself or the role of moving dislocations during creep must be considered when explaining the observable accelerating formation of the Z-phase in high-strength martensitic steel. Similar strain-assisted precipitation and growth phenomena are frequently observed during creep; for example, the sizes of the $\mathrm{M}_{23} \mathrm{C}_{6}$ carbides in a gauge portion in the crept specimens of Grade P92 are larger than those for a grip (Ennis, Zielinska-Lipiec, \& Czyrska-Filemonowicz, 2000; Ennis \& Czyrska-Filemonowicz, 2003). The sizes of the Laves phase in a gauge portion in ruptured specimens are larger than those for a grip in ruptured specimens of a low-carbon $9 \mathrm{Cr}-3 \mathrm{Co}-2 \mathrm{~W}-0.4 \mathrm{MoVNbMB}$ steel (Fedorova, Kipelova, Belyakov, \& Kaibyshev, 2013). Also, the number density of the MX carbides in a gauge portion is smaller than that for a grip during the creep of T91/MGC because of the consumption of MX due to the formation of the Z-phase (Sawada et al., 2006; 2011; 2014b).

Figure 26 illustrates feasible mechanisms to accelerate the boundary precipitation including the Z-phase. Lundin, Norell, Andren, and Nyborg (1997) showed the evidence for the latent creep resistance proposed by Glen (1958), which is a type of dynamic strain aging. They observed clusters of $\mathrm{Cr}, \mathrm{V}$, and $\mathrm{N}$ along a dislocation using an atom-probe field ion microscope in a creep-interrupted specimen of $11 \mathrm{Cr}-1 \mathrm{Mo}-1 \mathrm{~W}-\mathrm{VNbN}$ steel. A concept of the latent creep resistance is supported by Kubon, Foldyna, and Vodarek (1998), Kubon, Foldyna, Hajduk, and Simecek (2000), and Azuma, et al. (2002) as one of the strengthening mechanisms for high-strength martensitic steel. The rapid transportation of these segregated solute atoms along a dislocation to the grain boundaries by pipe diffusion is one of the potential mechanisms to increase the total amount of the solute atoms to be transported because the dislocations near the boundaries are possibly connected to the boundaries and many dislocations stagnate near the boundaries of the $\mathrm{Cr}-\mathrm{Mo}(\mathrm{W})$ steel when compared with those of the plain $\mathrm{Cr}$ steel 
(Abe et al., 1992).

Hayakawa, Terada, Yoshida, Nakashima, and Goto (2003a; 2003b) and Hayakawa, et al. (2007a; 2007b) showed applying the rapid stress change tests on several Cr-Mo steels that dislocations move dragging solute atoms, Mo, during creep and the dragging solute atoms is the rate-controlling process for the creep of Cr-Mo steels. They also suggested that the effective stress to the drag of solute atoms is estimated to be, at most, $10 \%$ of the applied stress, while the remaining is the back stress for the moving dislocations. The magnitude of the equivalent back stress can also be estimated in this study. However, the estimated back stress using the exponential law is significantly greater than the applied stress. There could be a clear gap between the above two lines of approach. However, there is no discrepancy, which will be explained in Appendix B. The solute atoms of Mo dragged by the dislocations arrive near the grain boundaries along with the dislocations during creep. When the dislocations are annihilated due to recovery, Mo atoms are segregated on the boundaries, which promotes the formation or growth of the Laves phase. The solute atoms of $\mathrm{W}, \mathrm{Cr}, \mathrm{V}, \mathrm{Nb}, \mathrm{C}, \mathrm{N}$, and $\mathrm{B}$ also interact with the dislocations. Hence, these solute atoms including Mo will potentially be swept out to the grain boundaries. Consequently, the formation or growth, or both, of the $\mathrm{Cr}_{23} \mathrm{C}_{6}, \mathrm{MX}$, the Laves phase, and the Z-phase are promoted on the boundaries during creep. There are numerous $\mathrm{Mo}, \mathrm{W}$, and $\mathrm{Cr}$ atoms in the matrix among the above-mentioned solute atoms; therefore, these atoms are scarcely exhausted in the accelerated segregation reactions on the boundaries. However, $\mathrm{Nb}$ and $\mathrm{V}$, which are the constituent elements of the Z-phase and the solubility limits for each are considered to be considerably small in high-Cr high-strength martensitic steels. Moreover, the total amount of $\mathrm{Nb}$ and $\mathrm{V}$ is not so large; thus, these elements are easily exhausted in the matrix. Therefore, to continue the accelerating formation of the Z-phase with increasing creep strain, a special mechanism other than pipe diffusion, lattice diffusion, and dragging of solute atoms by dislocations is required.

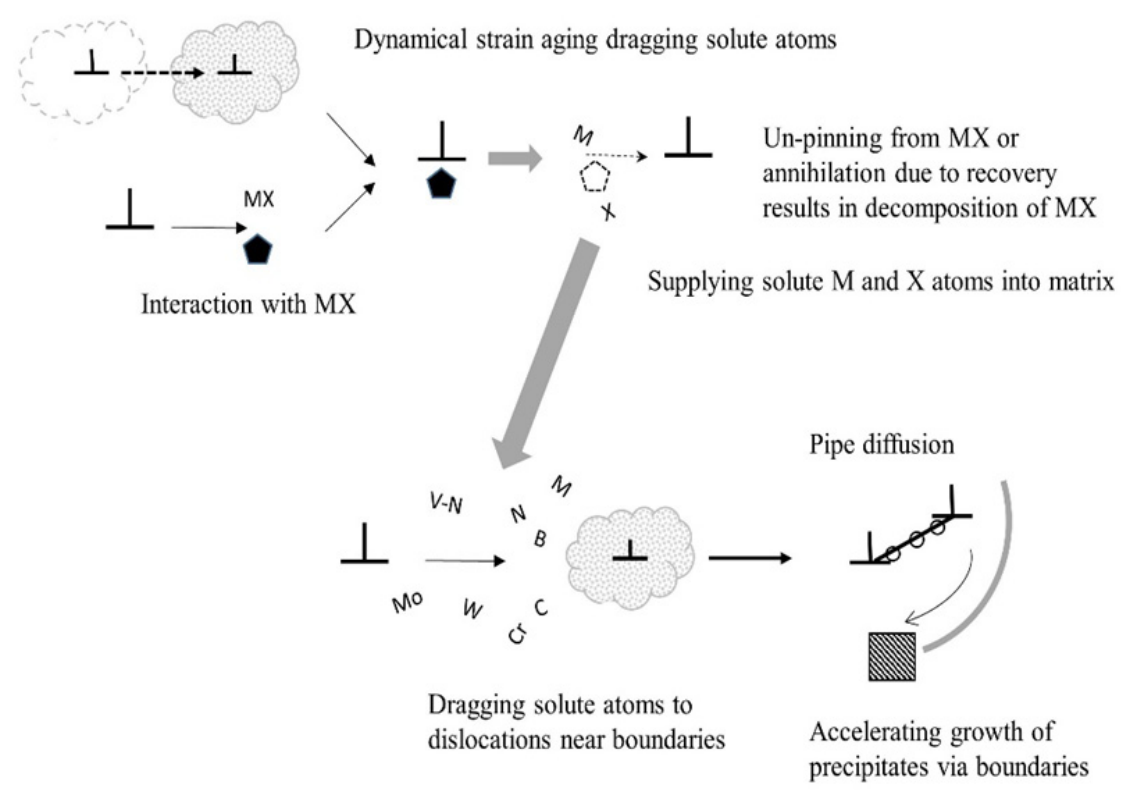

Figure 26. Schematic illustration for accelerating growth of boundary precipitates in high $\mathrm{Cr}$ martensitic steel with high strength

Tamura et al. (2004) observed that in a low-carbon $7 \mathrm{Cr}-0.4 \mathrm{~V}-0.09 \mathrm{~N}$ martensitic steel the soluble $\mathrm{V}$ in the matrix discontinuously increases, then decreases over a short time during tempering at $740-800{ }^{\circ} \mathrm{C}$ for up to $100 \mathrm{~h}$, even though the precipitation of $\mathrm{VN}$ is approximately complete after $20 \mathrm{~h}$ at least. A similar phenomena also occurs for $\mathrm{NbC}$ in a $9 \mathrm{Cr}-1 \mathrm{Mo}-0.18 \mathrm{~V}-0.085 \mathrm{Nb}$ system. Tamura, Nakamura, Shinozuka, and Esaka (2008) showed that in a low-carbon $7 \mathrm{Cr}-0.1 \mathrm{~V}-0.06 \mathrm{Nb}-0.077 \mathrm{~N}$ system, the amount of $\mathrm{MX}$ decreases gradually at the macroscopic scale during tempering at $750{ }^{\circ} \mathrm{C}$, whereas that of $\mathrm{Cr}_{2} \mathrm{~N}$ increases and the $\mathrm{V}$ and $\mathrm{Nb}$ contents in $\mathrm{MX}$ increase, whereas the $\mathrm{Cr}$ content does not. During the tempering of this steel up to $1000 \mathrm{~h}$, discontinuous changes in the chemical compositions of MX are clearly observed at least three times accompanying the discontinuous 
changes in the amount and the size. Here, the chemical composition of MX with the highest $\mathrm{Cr}$ content, i.e., $50 \mathrm{Cr}-35 \mathrm{~V}-15 \mathrm{Nb}$ in at\%, corresponds to that of the Z-phase (Suzuki et al., 2003; Sawada et al., 2006 and 2007). However, the MX particles with high $\mathrm{Cr}$ content have a cubic structure rather than a tetragonal structure. Therefore, the $(\mathrm{Cr}, \mathrm{M}) \mathrm{X}$ particles with high $\mathrm{Cr}$ content are not the Z-phase. Cipolla et al. (2010) demonstrated that the transient compound with the hybrid structure composed of cubic MX and the tetragonal Z-phase are formed in a $12 \% \mathrm{Cr}$ model steel during aging at $650{ }^{\circ} \mathrm{C}$ for up to $1000 \mathrm{~h}$. However, such compound is not reported during the tempering of the low-carbon $7 \mathrm{Cr}-0.1 \mathrm{~V}-0.06 \mathrm{Nb}-0.077 \mathrm{~N}$ steel. If the particles with $50 \mathrm{Cr}-35 \mathrm{~V}-15 \mathrm{Nb}$ were the Z-phase with tetragonal structure, the $\mathrm{Cr}$ content of the particles with $50 \mathrm{at} \% \mathrm{Cr}$ would not decrease, because the Z-phase with the tetragonal structure is the thermally stable phase.

The discontinuous changes in the precipitation reactions mentioned above are interpreted by combining the information on hardness and the analytical results of XRD. The MX particles formed on the sub-boundaries remain in the original positions for a while when the sub-grains are newly formed or grown during tempering. The isolated MX particles become energetically unstable and discontinuously dissolve into the matrix, then re-precipitate on nearby stable sites, such as dislocations. Similar processes are feasible to operate for MX particles precipitated on dislocations in a stress-free specimens because internal stresses always act on the dislocations, even in tempering. In that case, the change induced for each event may be too small to detect. However, in many cases there are numerous dislocations in martensitic steel to be annealed out to enable the changes to appear as though they occur continuously against time. Similar dissolution and re-precipitation of MX particles should occur, even for an extended period of creep time for the martensitic steel containing V and $\mathrm{Nb}$ because the applied stressed, except the internal stress, act on the moving dislocations during creep. In such cases where moving dislocations interact with $\mathrm{MX}$ particles, the soluble $\mathrm{V}, \mathrm{Nb}$, and $\mathrm{N}$ contents in the matrix can maintain a higher level for each, compared to those of the value calculated using a thermodynamic calculation software (Tamura et al., 2004). High levels of 0.15 and $0.02 \%$ for $\mathrm{V}$ and $\mathrm{Nb}$, respectively are confirmed after tempering a $7 \mathrm{Cr}-0.4 \mathrm{~V}-0.09 \mathrm{~N}$ system at $740{ }^{\circ} \mathrm{C}$ for $90 \mathrm{~h}$ and a $9 \mathrm{Cr}-1 \mathrm{Mo}-0.18 \mathrm{~V}-0.085 \mathrm{Nb}$ system at $740{ }^{\circ} \mathrm{C}$ for $4 \mathrm{~h}$, respectively (Tamura et al., 2004).

Furthermore, Tamura et al. (2011) also found a new and unusual phenomenon that some finely dispersed particles of a stable phase of $\mathrm{Ti}_{2} \mathrm{Y}_{2} \mathrm{O}_{7}$ are temporally decomposed in the matrix. Then, $\mathrm{TiO}_{2}$ and $\mathrm{Y}_{2} \mathrm{O}_{3}$ are formed when mechanically alloyed $\mathrm{Cr}-\mathrm{W}$ steel with $\mathrm{Ti}_{2} \mathrm{Y}_{2} \mathrm{O}_{7}$ is austenitized and then air-cooled. In these processes, $\mathrm{Ti}_{2} \mathrm{Y}_{2} \mathrm{O}_{7}$ is not thermally decomposed, but can be decomposed with the aid of dislocations that are introduced through the $\gamma$ to $\alpha$ transformation of the matrix. Again, fine particles of $\mathrm{Ti}_{2} \mathrm{Y}_{2} \mathrm{O}_{7}$ are reproduced after further tempering because the introduced dislocations are annealed out during tempering. These sequential reactions indicate that the existence of dislocations makes possible solute atoms in a supersaturated state to segregate on the dislocations.

Therefore, it is natural to consider that high levels of soluble $\mathrm{Nb}$ and $\mathrm{V}$ are maintained continuously during creep, because during creep fresh moving dislocations are always supplied, and so, sufficient amount of $\mathrm{Nb}$ and $\mathrm{V}$ can be always supplied on the boundaries to form Z-phase during creep. In summarizing this section, it can be said that when HRHD occurs in a sub-grain during the creep of high $\mathrm{Cr}$ martensitic steel containing $\mathrm{V}$ and $\mathrm{Nb}$, many dislocations introduced assist in transporting the solute atoms to the boundaries quickly through several processes, leading to the formation of coarse precipitates of the Z-phase, Laves phase, and $\mathrm{M}_{23} \mathrm{C}_{6}$.

\subsection{Roles of Precipitates}

\subsubsection{Laves Phase}

The formation of the Z-phase is undoubtedly responsible for the degradation in strength in Gr.IIIa and it is distinctly observed that the number density of the Z-phase increases with an increase in the creep strain due to consumption of the MX particles (Sawada et al., 2011). The trend in Table 17 shows that the larger the rupture elongation, the larger the degradation in strength for Gr.IIIa at $600{ }^{\circ} \mathrm{C}$. This trend is considered approximately accurate to some extent. However, as seen in Figure 22, we can confirm the distinct differences in the degradation in strength, even for a specific strain of $1 \%$. To explain the differences in the degree of degradation shown in Figure 22, it is essential for us to know a degree of strain within an HRHD zone, not a nominal strain, because at $1 \%$ strain, the $V$ for Gr.IIIa already begins to decrease and the HRHD is expected to begin as shown in Table 18. However, unfortunately, no investigations have been performed within an HRHD zone as yet. Therefore, until the aforementioned investigation can be performed, another solution is required to explain the degradation in strength. To solve this problem, we consider the hint that even though the nose temperatures of the TTP curve of the Z-phase for Grades T91 and T92 are similar $\left(\sim 650{ }^{\circ} \mathrm{C}\right)$, the average temperatures of Grs.IIIa of MJP and MJT are 634 and $622^{\circ} \mathrm{C}$, respectively, whereas that for T91/MGC is much lower $\left(583{ }^{\circ} \mathrm{C}\right)$, 
as shown in Table 18. Moreover, the average temperature of Gr.IIIa of Grade $91 / \mathrm{MgC}$ (Figure A8) is $581{ }^{\circ} \mathrm{C}$. There is a distinct difference between the average temperatures of Grs.IIIa between Grades 92 and 91, that is, Fe-Cr-W and Fe-Cr-Mo systems, respectively. The major differences in the chemical compositions of Grades 92 and 91 are due to the Mo and $\mathrm{W}$ contents, which are not considered to directly impact the formation of the Z-phase. Instead, it is more reasonable that the above-mentioned differences in the average temperature of Gr.IIIa are attributed to the formation temperature of the Laves phase in Grades 91 and 92. Tamura et al. (1988) reported the TTP curves for $\mathrm{Fe}_{2} \mathrm{Mo}$ and $\mathrm{Fe}_{2} \mathrm{~W}$ in equivalent Grade 91 steel and $8 \mathrm{Cr}-2 \mathrm{WVTa}$ steel plates, respectively. From the aforementioned curves, the nose temperatures for $\mathrm{Fe}_{2} \mathrm{Mo}$ and $\mathrm{Fe}_{2} \mathrm{~W}$ are 575 and $654{ }^{\circ} \mathrm{C}$, respectively. The information pertaining to the nose temperatures for the Laves phases, the average temperatures of Gr.IIIa for Grades 91 and 92, and the degradation in strength for Grs.IIIa of T91/MGC, Grade 91/MgC, T92/MJT, and P92/MJP are shown in Figures 12, 15, 18, 23, A4, and A6-A8. These figures suggest that the degradation in the long-term rupture strength of high-Cr high-strength martensitic steel is related to the coarsening of the Laves phases and the consumption of several MX particles due to the formation of the Z-phase. Figures 21 and 22 show that considerable degradation is observed for T91/MGC at a temperature of $600{ }^{\circ} \mathrm{C}$ ( $\sim$ several thousands of hours), which is higher than the nose temperature for $\mathrm{Fe}_{2} \mathrm{Mo}$. At high temperatures, the diffusion rate is generally high and the nucleation rate of precipitates is low. However, at low temperatures, the opposite is true. A nose temperature is determined by the diffusion-nucleation balance. These kinematical characteristics of the precipitation phenomena around the nose point of a TTP curve are well understood both experimentally and theoretically (Arai, 1970; Medina, 1997; Milkereit, Giersberg, Kessler, \& Schick, 2014). Therefore, when the Laves phase particles in T91/MGC readily grow and are coarsened at $600{ }^{\circ} \mathrm{C}$, grain boundary strengthening becomes incomplete, even after a small strain under a low stress. This causes HRHD and strain concentration to occur in an HRHD zone. Therefore, the vicious cycle of HRHD, the coarsening of the Laves phase, and the formation of the Z-phase by consuming MX particles occurs, even from a small nominal strain, which leads to serious degradation in strength for the Gr.IIIa of MGC at $600{ }^{\circ} \mathrm{C}$ compared to the P92/MJP.

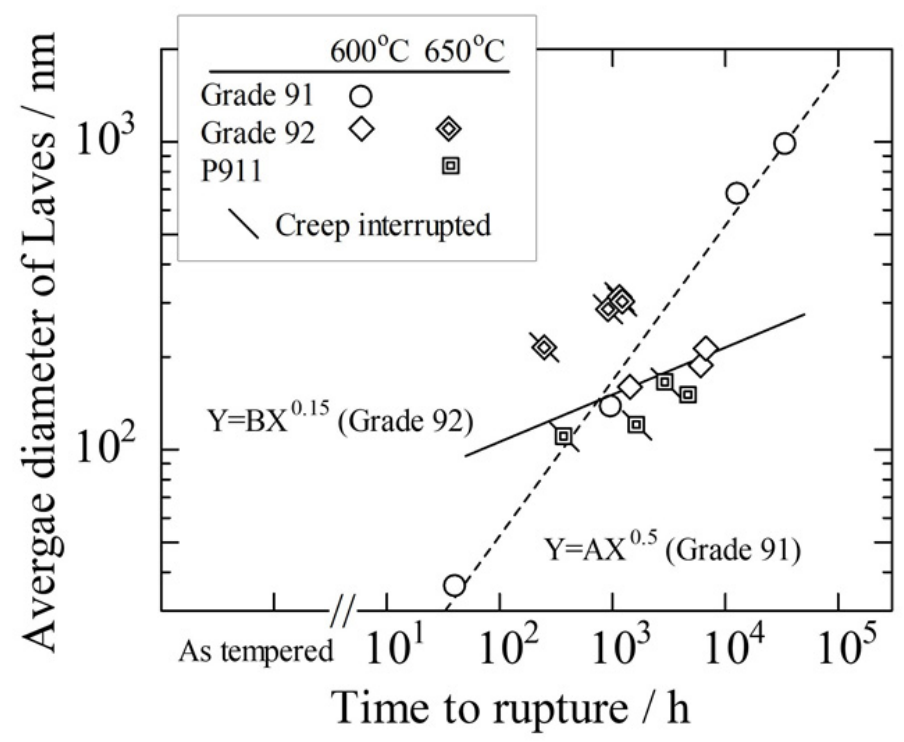

Figure 27. Average diameter of Laves phase in Grade 91, Grade 92, and P911 in a gauge portion of ruptured specimens. Creep interrupted specimens are also investigated and distinguished by thrash

Figure 27 shows the changes in the average diameter of the Laves phase observed in a gauge portion of ruptured specimens of Grade 91 (Suzuki et al., 2000) and Grade 92 (Jiang et al., 2013; Dudko et al., 2013; Nie et al., 2014). In the figure, creep-interrupted data are distinguished by thrash marks and the data at $650{ }^{\circ} \mathrm{C}$ for P911 (3Co) steel (Kipelova et al., 2012) are also added. The broken and solid lines in the figure represent the regression lines for Grade 91 and 92 at $600{ }^{\circ} \mathrm{C}$, respectively. Combining the data in the grip portions and aged specimens with the data for both the creep-interrupted and ruptured specimens, the following trends were deduced (the related figures are omitted for simplicity): i) The Laves phase is coarsened by increasing the aging 
temperature (Haetterstrand \& Andren, 2001), ii) straining promotes the growth of the Laves phase (Nie et al., 2014), and iii) the addition of boron suppresses the growth of the Laves phase (Azuma et al., 2002). As shown in Figure 27, the growth rate of the Laves phase (slope of the line) in Grade 92 appears to be lower than that in Grade 91 at $600{ }^{\circ} \mathrm{C}$, despite the lack of long-term data for Grade 92 . The growth rate of the Laves phase in Grade 92 at $600{ }^{\circ} \mathrm{C}$ is controlled by the diffusion rate of constituent elements at that temperature or $600{ }^{\circ} \mathrm{C}$, because the test temperature is below the nose temperature for $\mathrm{Fe}_{2} \mathrm{~W}\left(\sim 650{ }^{\circ} \mathrm{C}\right)$ and the diffusion rate of $\mathrm{W}$ in $\alpha-\mathrm{Fe}$ is slower than both that of Mo and the self-diffusion of $\alpha-\mathrm{Fe}$ (Oikawa, 1982). Therefore, the Laves phase particles of $\mathrm{Fe}_{2} \mathrm{~W}$ are finer than those for Grade 91. In addition, the total amount of Mo and $\mathrm{W}$ is approximately 1.4 times larger than that for Grade 91 in atomic ratio causes the clear Laves phase hardening observed in Gr.II of Grade 92. Also, in the case of Gr.IIIa of Grade 92, the strengthening effect due to the Laves phase may be observed at a small strain. Thus, HRHD is more difficult to achieve for Grade 92 than it is for Grade 91, which resulted in less degradation in strength for Grade 92, as seen in Figures 21 and 22.

From the above discussion, it is understood that the stability of the Laves phase under a given condition is considered to be the underlying cause for the breakdown in strength, even though the main reason is the partial consumption of MX due to the formation of the Z-phase. In other words, the growth and coarsening of the Laves phase triggers the beginning of HRHD, which should promote the formation of the Z-phase. The Laves phase shows a positive effect on grain boundary strengthening which delays the beginning of HRHD. However, the positive effect is easily lost when the Laves phase particles grow, which promotes the formation of the Z-phase and ultimately, the rapid degradation in strength. Therefore, it is very important to improve the stability of the Laves phase together with $\mathrm{M}_{23} \mathrm{C}_{6}$ to delay the occurrence of HRHD and the formation of the Z-phase. Hashizume et al. (2009) showed that a small addition of Re to $9 \mathrm{Cr}-4 \mathrm{~W}-3 \mathrm{Co}-\mathrm{NbVN}$ steel remarkably suppresses the growth of the Laves phase and increases the rupture strength at $650{ }^{\circ} \mathrm{C}$. Kunieda, Murata, Morinaga, and Koyama (2004) and Kunieda et al. (2006) suggest that Re retards the diffusion rate of $\mathrm{W}$ in $\alpha-\mathrm{Fe}$. The Laves phase in P911(3Co) is fine, even at $650{ }^{\circ} \mathrm{C}$, as shown in Figure 27. However, they did not completely discuss their results in their paper. Fedoseeva, Nikitin, Dudova, and Kaibyshev (2019) also showed that the addition of $0.17 \%$ Re to $10 \mathrm{Cr}-3 \mathrm{Co}-3 \mathrm{~W}$ martensitic steel is effective to suppress the coarsening rate of the Laves phase and the typical size is $200 \mathrm{~nm}$ in a ruptured specimen at $650{ }^{\circ} \mathrm{C}$ for $\sim 10000 \mathrm{~h}$. The temperature of $650{ }^{\circ} \mathrm{C}$ is close to the nose temperature for $\mathrm{Fe}_{2} \mathrm{~W}$; therefore, the growth rate may be predominantly controlled by the nucleation rate, rather than the diffusion rate at $\sim 650{ }^{\circ} \mathrm{C}$. Thus, it is considered that reducing the interfacial energy by controlling the amount of micro-alloying elements may be effective to stabilize the Laves phase for Grade 92 at $650{ }^{\circ} \mathrm{C}$. However, no literature on the aforementioned issue has been identified as yet.

\subsection{2 $\mathrm{M}_{23} \mathrm{C}_{6}$}

$\mathrm{M}_{23} \mathrm{C}_{6}$ particles formed on the PAGBs and sub-boundaries play an important role in maintaining the strength of martensitic steel and preventing the growth of sub-grains. $\mathrm{M}_{23} \mathrm{C}_{6}$ particles remain moderately sized throughout long-term creep deformation using the DFRP phenomena highlighted in Sections 4.1-4.5. Apart from the stability of the Laves phase, the DFRP reaction of $\mathrm{M}_{23} \mathrm{C}_{6}$ is very effective in delaying the occurrence of HRHD around the strain for $Q_{\max }$. Figure 28 shows the relationship between the size of $\mathrm{M}_{23} \mathrm{C}_{6}$ and the time to rupture for Grades 91 and 92 . The data shown in the figure with thrash marks are the results from the creep-interrupted specimens (Dudko et al., 2013). The size of $\mathrm{M}_{23} \mathrm{C}_{6}$ for a creep-interrupted specimen is between that for a gauge and a grip of the ruptured specimen because the size in a ruptured specimen is larger than that of a grip (Haetterstrand \& Andren, 2001; Ennis \& Czyrska-Filemonowicz, 2003; Dimmer et al., 2003, Dudko et al., 2013). Nie et al. (2014) reported the size of the $\mathrm{M}_{23} \mathrm{C}_{6}$ of Grade P92 ruptured at $700{ }^{\circ} \mathrm{C}$, but since the exact time to rupture could not be ascertained, the data was omitted. The lines for Grades 91 and 92 are guidelines for a lower limit; these are not the regression lines. Under the assumption that all data are valid, the following were deduced because there was no data directly comparing Grades 91 and 92 presented in a single paper: i) the size at $650{ }^{\circ} \mathrm{C}$ is coarser than that at $600{ }^{\circ} \mathrm{C}$, except for the data reported by Jiang et al. (2013), ii) the time exponent for Grade $92(n=0.16)$ is smaller than that for Grade $91(n=0.2)$, and iii) the growth or coarsening rate, or both, for Grade 92 appears slower than that for Grade 91, which may be due to the W and B contents in Grade 92. However, the difference between the time exponents $n=0.16-0.2$ may not be as significant because of the large scattering for both steels. We can observe the large difference between the time exponents of Grades 91 and 92 for the Laves phase, as shown in Figure 27, because the rate-determining processes for the growth or coarsening of the Laves phase for these steel differ from each other, as mentioned in the previous section. However, the rate-determining process for $\mathrm{M}_{23} \mathrm{C}_{6}$ is the same at $\sim 600{ }^{\circ} \mathrm{C}$ and the diffusion of constituent elements is a rate-controlling process because the nose temperature of the corresponding TTP curve is higher than the tempering temperature. Thus, from the above-mentioned metallurgical considerations, the time exponent 
for Grade 92 may be slightly smaller than that for Grade 91 . The size of the $\mathrm{M}_{23} \mathrm{C}_{6}$ formed on the PAGBs is considerably larger than that on the sub-boundaries (Abe, 2008; 2009), but the variations in the size of the $\mathrm{M}_{23} \mathrm{C}_{6}$ systematically investigated for Grades 91 and 92 during creep are not yet ascertained.

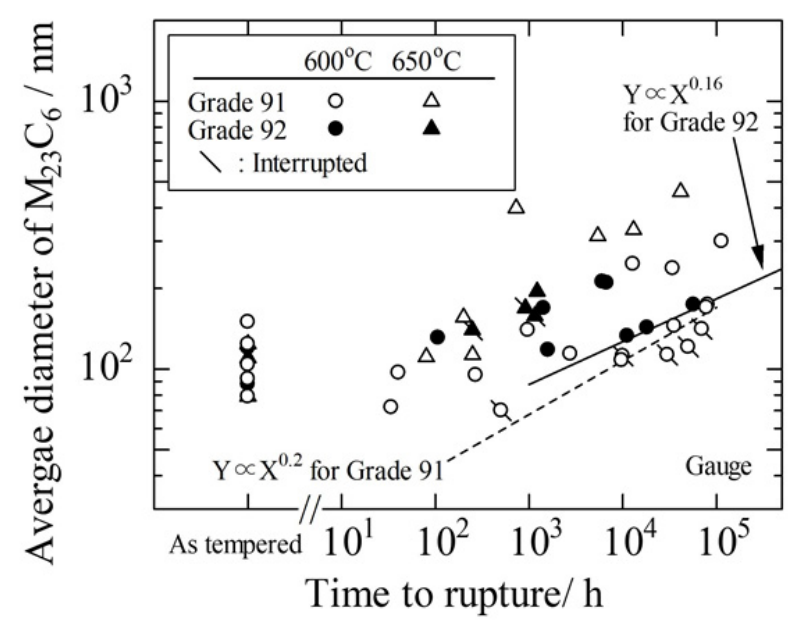

Figure 28. Relationship between time to rupture and average diameter of $\mathrm{M}_{23} \mathrm{C}_{6}$ formed at 600 and $650{ }^{\circ} \mathrm{C}$ in Grades 91 and 92 steel. Slashed marks correspond to creep interrupted specimens and the data by Jiang et al. (2013) are marked by gray, the details of which are explained in the text. A dotted and real lines are drawn for the lower limits to Grade 91 and 92, respectively, using naked eyes

$\mathrm{Cr}_{23} \mathrm{C}_{6}$ is considered to be stabilized by dissolving Fe, Mo, and W (Yi, Xu, Xia, Gang, \& Chen, 2017; Sanhueza et al., 2019). The diffusion of the constituent elements of the $\mathrm{M}_{23} \mathrm{C}_{6}$ particles is required for the nucleation and growth of the particles. The diffusion rate in $\alpha-\mathrm{Fe}$ at $\sim 600{ }^{\circ} \mathrm{C}$ increases in the order of $D_{M o}>D_{C r}>D_{F e}>$ $D_{W}$, where the suffices denote each constituent element (Oikawa, 1982). Therefore, Mo may not suppress the growth of $\mathrm{M}_{23} \mathrm{C}_{6}$ through a diffusion process. However, Mo has been utilized in many heat-resistant steel for an extended duration; thus, Mo appears to stabilize $\mathrm{M}_{23} \mathrm{C}_{6}$ through a nucleation process. On the other hand, $\mathrm{W}$ definitely suppresses the growth rate of $\mathrm{M}_{23} \mathrm{C}_{6}$ because the diffusion of $\mathrm{W}$ is slower than other constituent elements of $\mathrm{M}_{23} \mathrm{C}_{6}$. Therefore, the growth and coarsening rates of $\mathrm{M}_{23} \mathrm{C}_{6}$ in Grade 92 are expected to be slower than those in Grade 91. Yoshizawa, Igarashi, and Nishizawa (2005) showed that the coarsening rate of $\mathrm{M}_{23} \mathrm{C}_{6}$ in the W-containing martensitic steel is lower than that of W-free steel; they explain this phenomenon semi-quantitatively by assuming Ostwald ripening (Lifschitz \& Siyozov, 1961; Wagner, 1961).

The average chemical composition of the $\mathrm{M}_{23} \mathrm{C}_{6}$ of ruptured specimens for Grade 91 at $600{ }^{\circ} \mathrm{C}$ is 64.4Cr-21.8Fe-9.9Mo-NbVSi. $\mathrm{Cr}$ is slightly enriched during long-term tests, but the compositional range varies steadily during creep (Suzuki et al., 2000; Kimura et al., 2002). On the other hand, the chemical composition of $\mathrm{M}_{23} \mathrm{C}_{6}$ in Grade 92 is rarely reported: $46.1 \mathrm{Cr}-23.6 \mathrm{Fe}-27.3 \mathrm{~W}-2.2 \mathrm{Mo}-0.9 \mathrm{~V}$ at $600{ }^{\circ} \mathrm{C}$ for $t_{r}=9755 \mathrm{~h}$ (Ennis et al., 1997), 6.3Cr-8.7Fe-46.3W-7.7Mo at $600{ }^{\circ} \mathrm{C}$ for $t_{r}=8472 \mathrm{~h}$ (Nie et al., 2014), and $63 \mathrm{Cr}-19 \mathrm{Fe}-12 \mathrm{~W}-4.4 \mathrm{Mo}$ aged at $700{ }^{\circ} \mathrm{C}$ for $2000 \mathrm{~h}$ (Gao et al., 2017). This seems to imply that the compositional range of $\mathrm{M}_{23} \mathrm{C}_{6}$ in Grade 92 could be wider than that for Grade 91 because of the micro-segregation of W. Maruyama, Sawada, and Koike (2001) reported that the addition of $\mathrm{Ni}$ to $12 \mathrm{Cr}-0.5 \mathrm{Mo}-\mathrm{VNb}$ steel increases the size of the $\mathrm{M}_{23} \mathrm{C}_{6}$ particles during creep at $600{ }^{\circ} \mathrm{C}$. Ni is not a constitutional element of $\mathrm{M}_{23} \mathrm{C}_{6}$. Hence, this phenomenon can be explained as follows: The addition of Ni decreases an $\alpha-\gamma$ transformation temperature (Fujita, Yamashita, \& Miyake, 1980), which promotes the recovery and diffusion of the matrix. Therefore, the size of $\mathrm{M}_{23} \mathrm{C}_{6}$ increases with the addition of Ni. However, there is no report on the effect of a small amount of $\mathrm{Ni}$ on the size of $\mathrm{M}_{23} \mathrm{C}_{6}$ in Grades 91 and 92 . The addition of small amounts of boron to high-Cr martensitic steel is confirmed to suppress the coarsening of $\mathrm{M}_{23} \mathrm{C}_{6}$ and improve the rupture strength (Azuma et al., 2002; Danielsen, 2007; Abe, 2008, 2009, 2011). This technique is explained as follows: After boron is segregated on grain boundaries during normalizing, the segregated boron atoms on the PAGBs move towards the newly formed $\mathrm{M}_{23} \mathrm{C}_{6}$ during tempering and stabilize with the $\mathrm{M}_{23} \mathrm{C}_{6}$ particles for an extended duration (Abe, 2008, 2009, 2011; Liu, Fors, Golpayegani, Andren, \& Wahnstrom, 2012). However, majority of the boron in the typical Grade 92 is used to form massive BN particles, which reduce the reduction of area, as shown in Figures 12 and 15. Therefore, boron is not used effectively in Grade 92. Liu et al. 
(2012) and Sanhueza et al. (2019) attempted to explain the time rule for the precipitated $\mathrm{M}_{23} \mathrm{C}_{6}$ during the creep of martensitic steel containing boron, based on Ostwald ripening; unfortunately, they were unsuccessful in their attempts. The time exponents shown in Figure 28 are approximately close to $1 / 3$, but this does not necessarily indicate that the coarsening process is Ostwald ripening. The diffusion of the constitutional elements of $\mathrm{M}_{23} \mathrm{C}_{6}$ is very important for the growing process of $\mathrm{M}_{23} \mathrm{C}_{6}$ because the creep temperature of martensitic steel is far below the nose temperature of the respective TTP curve of martensitic steel. However, the $\mathrm{M}_{23} \mathrm{C}_{6}$ during the creep of martensitic steel is in a nucleation and growth process, as opposed to in a simple coarsening process. This lends credibility to the DFRP process of $\mathrm{M}_{23} \mathrm{C}_{6}$ on the sub-boundaries during creep, as explained in Section 4.1.3, as well as the rearrangement of the $\mathrm{M}_{23} \mathrm{C}_{6}$ arising from HRHD, which occurs from the early stage of creep as explained in Sections 4.1-4.5. Therefore, the average composition of $\mathrm{M}_{23} \mathrm{C}_{6}$ should continuously change during creep, though the experimental results for Grade 92 have not been obtained. Moreover, the segregation of boron onto $\mathrm{M}_{23} \mathrm{C}_{6}$ is presumed to occur after the nucleation of $\mathrm{M}_{23} \mathrm{C}_{6}$ from the boron profile across an $\mathrm{M}_{23} \mathrm{C}_{6}$ particle (Abe, 2011). These facts indicate that the interfacial energy during creep is not considered to be as constant as the Ostwald ripening in liquids.

\subsection{Variations in Creep Curve}

\subsubsection{Comparison between Grade 91 and Grade 92}

As discussed in Section 5.1, the rupture strengths of Grade $92\left(\sim 600^{\circ} \mathrm{C}, 100000 \mathrm{~h}\right)$ are larger than those of Grade 91 , even upon neglecting the heat-to-heat variation in strength. These differences are caused by the consumption of finely dispersed MX particles due to the formation of the Z-phase, as indicated by Sawada et al. (2011). However, in Sections 5.2 and 5.3.1, this difference in strength, i.e., the amount of Z-phase formed at $600{ }^{\circ} \mathrm{C}$ for longer than several tens of thousands hours, is not directly related to the rupture elongation, but with the alloy system itself, i.e., $\mathrm{Fe}-\mathrm{Cr}-\mathrm{W}$ or Fe-Cr-Mo system. At $\sim 600{ }^{\circ} \mathrm{C}$, the $\mathrm{Fe}_{2} \mathrm{Mo}$ particles are easily coarsened compared to $\mathrm{Fe}_{2} \mathrm{~W}$ particles (Figure 27), which causes the HRHD to initiate at a smaller strain, consequently forming a larger amount of the Z-phase. Figure 29 shows the relationship between the average normalized strain rate and the strain for Gr.IIIa of the four steels of Grades 91 and 92. Clear differences in the transient creep are confirmed between Grades 91 and 92. The average NSRs for Grade 91 are smaller than those for Grade 92 in transient creep, which indicates that Grade 91 is more easily hardened than Grade 92 . This is because the formation of the Laves phase particles is faster in Grade $91\left(\mathrm{Fe}_{2} \mathrm{Mo}\right)$ than that in Grade $92\left(\mathrm{Fe}_{2} \mathrm{~W}\right)$ in transient creep. In accelerating creep, the average normalized creep rates through 1-5\% for Grade 91 appear slightly larger than those for Grade 92 . This can be explained as follows: Table 18 shows that in an Fe-Cr-Mo system (Grade 91) finer Laves phase particles are readily formed and $Q V C_{\max }$ is obtained at a smaller strain followed by the decrease in $Q$ and the occurrence of HRHD accompanying a faster growth of the Laves phase (see Figure 27). This causes a faster formation of the Z-phase and a faster recovery, even in the transient creep of Gr.IIIa, when compared with those for an Fe-Cr-W system (Grade 92). These sequential phenomena are passed more severely from the transient creep to the accelerating creep, and ultimately, the time to rupture for Grade 91 becomes shorter than that of Grade 92.

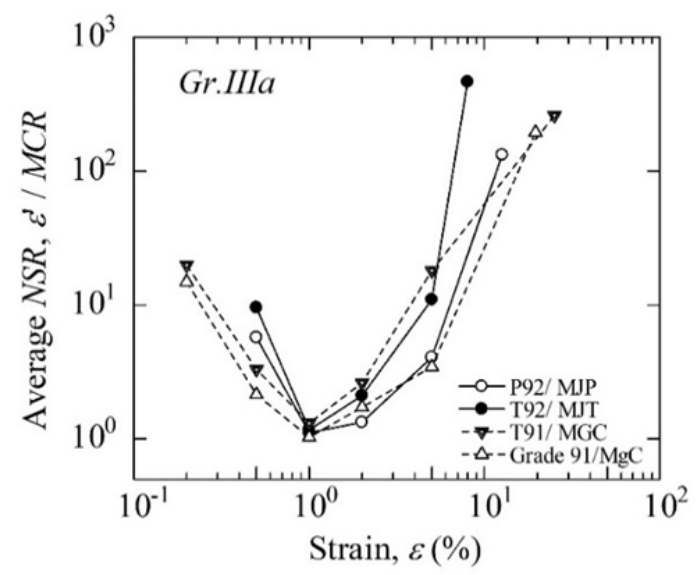

Figure 29. Comparison among the average NSRs for each Gr.IIIa of P92/MJP, T92/MJT, T91/MGC, and Grade $91 / \mathrm{MgC}$ as functions of creep strain 


\subsubsection{Heat-to-Heat Variations}

\section{1) Grade 92}

The rupture behavior at $600{ }^{\circ} \mathrm{C}$ for P92/MJP and T92/MJT (Figure 21) differ from each other. Although the rupture stresses for Grs.I and II of MJT are higher than those of MJP, the degradation in strength (the slope of the regression line) in Gr.III for MJT is apparent, and the rupture strength for Gr.IIIa of MJT tends to be smaller than that of MJP. From the explanation in Sections 4.3, 4.4, and 5.1, the higher rupture stresses for Grs.I and II of MJT may be caused by the higher initial hardness and the higher formability of the Laves phase due to higher Si content (Hosoi et al., 1986; see also Table 3) when compared with the case of MJP. A severe degradation in strength then occurs in Gr.IIIa because the Laves phase particles once formed are easily coarsened, thus triggering an HRHD process and a subsequent Z-phase formation. These degradation behaviors can also be traced in Figure 29. The slope of MJT in Figure 29 is steep in the transient creep or the initial hardening rate is high, but in acceleration creep the slope increases with increasing strain. When $V$ begins to decrease, HRHD begins to occur and then the Z-phase is easily formed in an HRHD zone, as explained in Section 5.2.2. Therefore, the changes in $V$ are an indicator of the formation of the Z-phase. Figure 30 shows the relationships between $V$ and the strain for Gr.IIIa of P92/MJP and MJT. From the figure it is easily inferred that the amount of Z-phase formed in MJT appears larger in accelerating creep than that of MJP, which results in a more severe degradation in strength for MJT when compared with that in MJP. Therefore, it is desirable to reduce the initial hardness (Table 3) as well as the Si content as low as possible, in an effort to mitigate the degradation in strength.

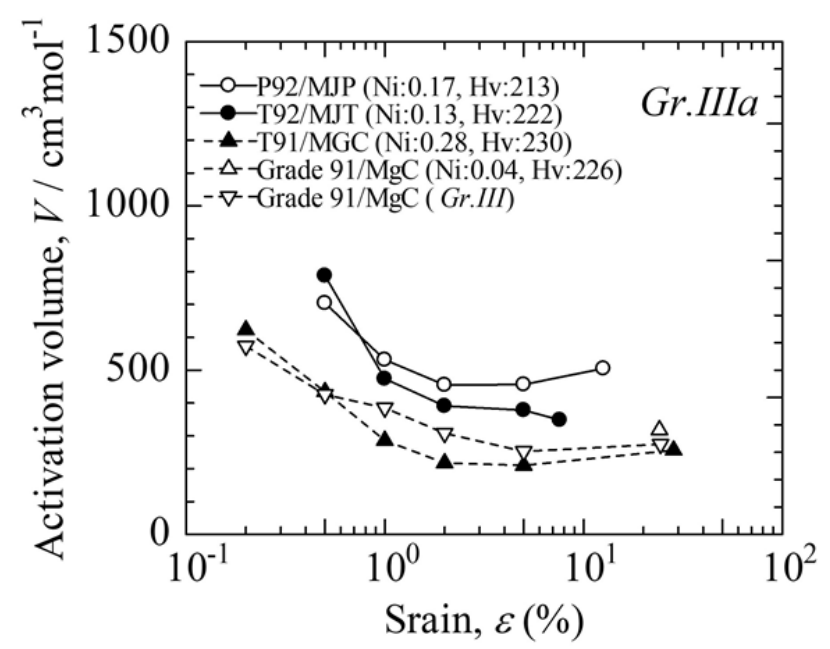

Figure 30. Changes in the activation volume as functions of creep strain for Gr.IIIa of MJP, MJT, MGC, and $\mathrm{MgC}$. Ni content is presented in per cent. Data for Gr.III of Grade 91/MgC are also plotted for reference, because the data for Gr.IIIa is limited only for rupture data

\section{2) Grade 91}

It is not easy to mitigate the degradation in the long-term rupture strength of Grade 91 steel because the Laves phase in an Fe-Cr-Mo system is easily coarsened at $\sim 600{ }^{\circ} \mathrm{C}$ than that in an Fe-Cr-W system. However, Grade 91 is more attractive from the economical point of view. Among the Grade 91 steels investigated, the rupture strength of $\mathrm{MgC}\left(\sim 60{ }^{\circ} \mathrm{C}, 100000 \mathrm{~h}\right)$ is higher than that of MGC, as shown in Figure 21 and Table 16. Sawada et al. (2014a) explained this phenomenon as the lower formation of the Z-phase due to the smaller amount of $\mathrm{Ni}$ in $\mathrm{MgC}$ than that in MGC. The creep data obtained under $110 \mathrm{MPa}$ for $t_{r}=35420 \mathrm{~h}$ are the longest among the creep curves at $600{ }^{\circ} \mathrm{C}$ for $\mathrm{MgC}$ and the corresponding data of $\mathrm{MGC}$ is obtained under $100 \mathrm{MPa}$ for $t_{r}=34151 \mathrm{~h}$. Figure 31 shows the relationship between the normalized creep rate by $M C R$ and strain for these two conditions. During transient creep, the normalized strain rates of both behave similarly, but during accelerating creep, the normalized creep rate for MGC is not only larger, but the slope is steeper when compared to those for $\mathrm{MgC}$. Although it is reported that $\mathrm{Ni}$ in martensitic steel promotes the formation of the Z-phase (Danielsen \& Hald, 2009; Yan et al., 2013; Sawada et al., 2014a), the mechanisms for the Ni effect have not been elucidated. It may be possible that the addition of Ni merely promotes the recovery rate of the martensitic matrix 
as highlighted in Section 5.3.2. In either case, the higher Ni content of MGC is responsible for the steeper creep rate in accelerating creep shown in Figure 31. In addition to this, the stress relief annealing at $730{ }^{\circ} \mathrm{C}$ was performed after tempering at $760{ }^{\circ} \mathrm{C}$ for $\mathrm{MgC}$. Consequently, the fact that the initial hardness of $\mathrm{MgC}$ is softer than that of MGC should be also taken into consideration as a potential reason for the prolonged rupture lives of $\mathrm{MgC}$ under long-term creep tests at $\sim 600^{\circ} \mathrm{C}$.

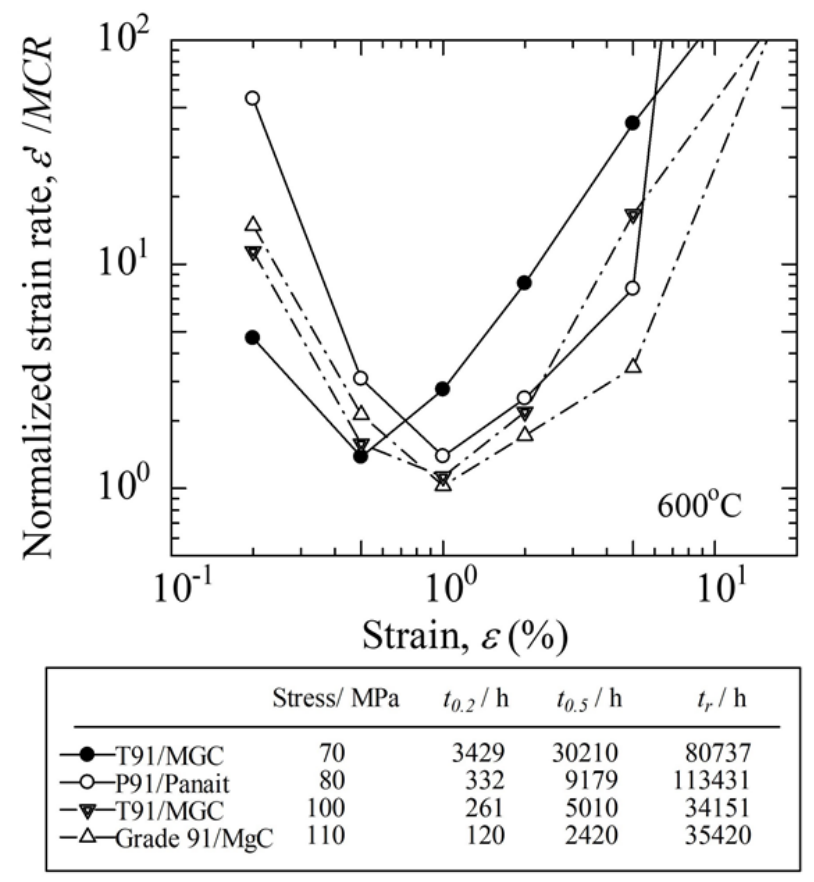

Figure 31. Relationship between strain and normalized strain rate for T91/MGC, P91/Panait, and Grade 91/MgC under the indicated stress at $600^{\circ} \mathrm{C}$

The rupture data of P91/Panait at $600{ }^{\circ} \mathrm{C}$ under $80 \mathrm{MPa}$ and $t_{r}=113431 \mathrm{~h}$ appears comparable to the linearly extrapolated data for Grade 91/MgC with low-Ni content, as shown in Figure 21. This data is very valuable as it is the longest published data. The corresponding long term data form the NIMS data sheets is $t_{r}=80737 \mathrm{~h}$ for T91/MGC at $600{ }^{\circ} \mathrm{C}$ under $70 \mathrm{MPa}$. The relationship between the normalized strain rate and the strain for both steels is also shown in Figure 31. These data for both steels are scanned from the figures in published papers (Panait et al., 2010b) and then the creep curves of the $\varepsilon-t$ and $\varepsilon-\dot{\varepsilon}$ relationships were re-constructed; therefore, the accuracy of the curves may not be high, particularly in the low and high strain ranges. However, it is clearly seen in Figure 31 that there is a large difference between their creep behaviors. The normalized strain rate of $\mathrm{P} 91 / \mathrm{Panait}$ is higher during transient creep than that of T91/MGC and the strain rate continues to decrease up to $1 \%$ strain. The strain rate in accelerating creep of P91/Panait is considerably smaller than that of MGC, although the accelerating creep of MGC begins from $0.5 \%$ strain. It may be suggested from these characteristics of the creep curve of P91/Panait that the Laves phase is hardly formed or coarsened, the degree of HRHD is small, and therefore, the formation of the Z-phase and the consequent consumption of MX are delayed. This may be the reason for the prolonged rupture life of P91/Panait compared to that of T91/MGC. This is supported by experimental observations that the Z-phase is merely observed in the ruptured specimen for $113431 \mathrm{~h}$ and the MX particles still trap the dislocations (Panait et al., 2010b). The chemical composition of P91/Panait (0.1C-0.36Si-0.41Mn-0.015P-0.003S-0.11 Ni-8.43Cr-0.92Mo-0.20V-0.068 Nb-0.059N-0.022Al) is similar to those of MGC and $\mathrm{MgC}$, except that it contains slightly lower $\mathrm{Ni}$ and slightly higher $\mathrm{N}$ compared to MGC. Although the normalizing temperature for both steels are the same $\left(1050{ }^{\circ} \mathrm{C}\right)$, the tempering methods are different. T91/MGC is tempered at $765{ }^{\circ} \mathrm{C}$, whereas $\mathrm{P} 91 /$ Panait is tempered at $750{ }^{\circ} \mathrm{C}$ after pre-tempering at $735^{\circ} \mathrm{C}$, but the initial hardness is of the latter is unknown. Instead, Panait investigated the dislocation density and the sub-grain size of initial and ruptured specimens (Panait et al., 2010b). The results are compared with those for T91/MGC including creep interrupted specimens (Sawada et al., 2011) and are shown in Figure 32. It is 
difficult to accurately evaluate the data in the ruptured specimens for both because both parameters may be affected by the rupture elongation. However, the figure indicates that there are distinct differences in the dislocation density and sub-grain size in their initial states. The sub-grain size of P91/Panait is considerably smaller than that of T91/MGC, even though the dislocation density is lower than that of T91/MGC. The reason for the relationship between these values and heat treatment is unclear, but the relationship between the initial microstructure or the microstructures within an early stage of transient creep and long-term rupture strength should be thoroughly investigated in the future.

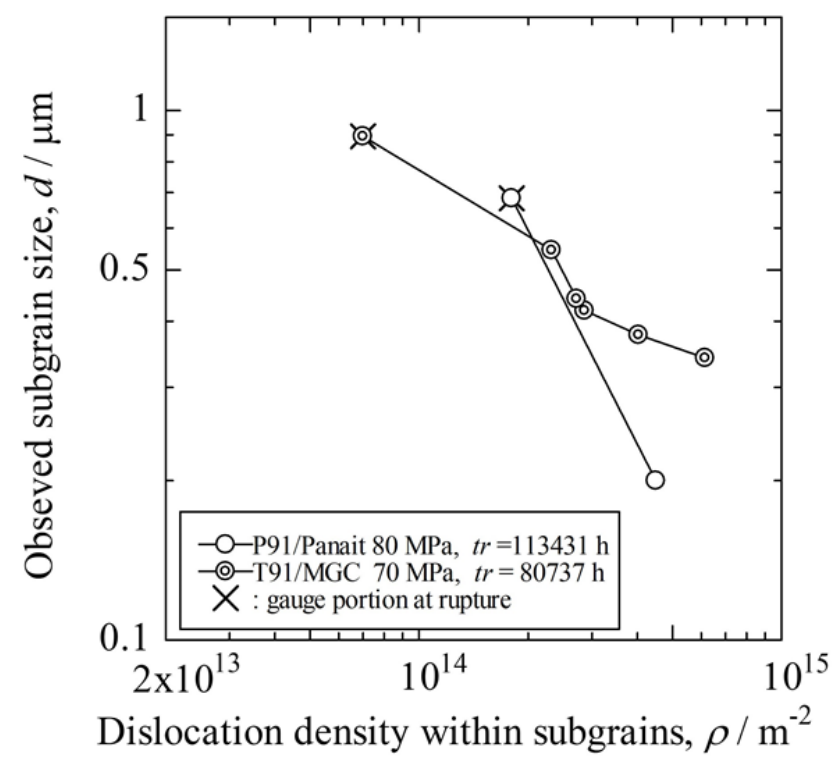

Figure 32. Relationship between dislocation density and sub-grain size for Grade 91 during creep at $600{ }^{\circ} \mathrm{C}$

Regarding the changes in the microstructure during transient creep, the observation of misorientation using an electron backscattered diffraction technique may be useful. Komazaki (2009) referred to unpublished work by Dr. Mitsuhara and indicated that the frequency of lath martensite with a misorientation of $0.5^{\circ}$ at a very early stage during the creep of $9 \mathrm{Cr}-1 \mathrm{Mo}$ steel changes considerably. However, the studies on the control of microstructure at the beginning of creep is limited.

Nevertheless, there may still be numerous potential techniques to improve the long-term rupture strength of high-strength $\mathrm{Fe}-\mathrm{Cr}$-Mo steel to mitigate rapid hardening during transient creep and to delay the recovery during accelerating creep, other than reducing $\mathrm{Ni}, \mathrm{Al}$, and the degree of segregation and controlling heat treatment (Sawada, Suzuki, Kushima, Tabuchi, \& Kimura, 2008; Sawada et al., 2014a; 2019a; Sawada \& Kimura, 2019b). If new techniques are explored, these may also be applicable to Fe-Cr-W systems. The control of the initial microstructure (explained by Figure 32), particularly hardness, is considered to be important.

\subsection{On-Line Prediction of Degradation Employing QVC Analysis}

As shown in Figure 21, when we analyze the rupture strength using an exponential law like Equation 1 assuming a thermally activated process for creep, the linear applied stress of high-Cr martensitic steel approximately decreases in an S-shape manner with increasing logarithms of time to rupture at $\sim 600{ }^{\circ} \mathrm{C}$. Severe degradation in strength is observed in the time zone approaching $100000 \mathrm{~h}$, where it is very important to determine the maximum allowable tensile stresses at high temperatures to obtain a $100000 \mathrm{~h}$ rupture strength. The trend of degradation in strength beyond $100000 \mathrm{~h}$ is expected to be mitigated based on metallurgical considerations explained in Sections 4.3.7, 4.4.7, and 4.5.7. However, we require long-term rupture data for $\sim 70000 \mathrm{~h}$ to obtain the $100000 \mathrm{~h}$ rupture strength of Grades 91 and 92 with high accuracy (Tamura, 2015a). This is because it is very difficult to estimate the changes in microstructure beyond tens of thousands of hours including the amount of MX particles consumed or the Z-phase formed. If we can estimate the risk of degradation in the strength of the martensitic steel within a short time period, we can focus on the appropriate countermeasures at an earlier stage than that of the conventional estimation method. As mentioned in Sections 4.3-4.5 and Figures A4, A6, and A7, we can confirm a similar degradation relationship between stress and time to $0.5-1 \%$ strain with the 
degradation in rupture strength. If such an estimation is required, it is recommended to collect the creep data at $\sim 600{ }^{\circ} \mathrm{C}$ under the stresses aiming at $100000 \mathrm{~h}$ to rupture for Grade 91 and at $\sim 625{ }^{\circ} \mathrm{C}$ for Grade 92 . In the case of Grade 92, it is possibly expected from Figures 12, 14, 15, 17, A4, and A6 by analyzing a set of creep curves using the QVC method introduced in Section 2 that $Q_{\max }$ is observed within $0.5 \%$ strain or $t_{0.5}<5000 \mathrm{~h}$ and then a sign for recovery is detected. The variations in $V$ after $V_{\max }$ inform us of the degree of degradation as seen in Figure 30. Moreover, the tendency of the degradation in long-term rupture strength under a creep condition of Gr.IIIa should possibly be obtained within 5000-10 $000 \mathrm{~h}$, because such a tendency signals the beginning of accelerating creep if the terms $\left[V_{s}\right]+C_{s}$, not $\left[Q_{s}\right]$, control the creep rate in transient creep as explained in Tables 11 and 14. In other cases, it is also a high risk for the degradation in rupture strength under the condition of Gr.IIIa, when the time to $0.5 \%$ strain is shorter than the estimated value obtained by using the QVC analysis for the data corresponding to Gr.III. By continuing such processes of trial and error, we can efficiently select an appropriate combination of a production route, chemical composition, microstructure, and hardness, so as to delay or mask the Gr.IIIa region. Although the solution method is not perfect, it enables a solution to be obtained within a short period of time $(<10000 \mathrm{~h})$. Moreover, it is the main advantage of using the $Q V C$ analysis because we can obtain the results using the numerical data of creep curves usually recorded online without interrupting a set of creep tests. On the other hand, it is hoped that the degradation in strength does not continue indefinitely along the extrapolation line for Gr.IIIa. It is possible that in actual power plants, sub-grains grow gradually and consequently the harmful effects of HRHD will be mitigated, as shown in Figures 12,15 , and 18 .

Moreover, Zeng et al. (2018), by analyzing the used reheater pipe of Grade P92 serviced at $603{ }^{\circ} \mathrm{C}$ under $5.87 \mathrm{MPa}$ for $9854 \mathrm{~h}$, confirmed that the fine Laves phase particles of the order of $100 \mathrm{~nm}$ are formed inside the lath martensite together with the Laves phase on the boundaries. Such finely dispersed Laves phase particles inside the lath martensite have not been reported in ruptured, creep-interrupted, or aged specimens. It is very important to periodically investigate the changes in the microstructure of the used components in power plants. A similar systematic cost-effective investigation is also possible in laboratories, which contributes to the lifespan and longevity of the technological components of power plants. In such cases, if we used a single specimen with different diameters for creep tests, we can systematically and economically obtain valuable information on the changes in microstructure during long-term services under power plant conditions.

\section{Conclusions}

The long-term creep curves of Grades T91, T92, and P92 along with the laboratory-prepared $9 \mathrm{Cr}-1 \mathrm{~W}$ and $9 \mathrm{Cr}-4 \mathrm{~W}$ steels were analyzed by applying an exponential law to the test temperature $(T)$, stress $(\sigma)$, and time to rupture $\left(t_{r}\right)$ or time to a specific strain $\left(t_{\varepsilon}\right)$. The observed creep variables of $T, \sigma$, and $t_{r}$ or $t_{\varepsilon}$ are converted to the activation energy $(Q)$, the activation volume $(V)$, and the Larson-Miller constant $(C)$, without using any adjustable parameters. The variations in $Q, V$, and $C$ as functions of creep strain are discussed metallurgically and the following conclusions are obtained:

1) Sub-grain boundary strengthening by the swept out dislocations (SBSD) is an essential process at the initial stage of the creep of martensitic steel.

2) Hardening by the dissolution and re-precipitation of fine $\mathrm{M}_{23} \mathrm{C}_{6}$ particles and the precipitation of the Laves phase are added to the SBSD for high-Cr high-strength martensitic steel, which increases $Q$.

3) After the peaks of $Q, V$, and $C$, the heterogeneous recovery and simultaneously occurring heterogeneous deformation (HRHD) should begin at an early stage of transient creep in the vicinity of several of the weakest boundaries due to coarsening of the precipitates, which triggers an unexpected degradation in strength due to the accelerated formation of the Z-phase consuming the MX particles. The microstructural investigation in the HRHD zone, which is expected to occur in the later stage of transient creep, is an issue for future investigation.

4) Stabilization of $\mathrm{M}_{23} \mathrm{C}_{6}$ and the Laves phase is important to mitigate the degradation in the long-term rupture strength of high-strength martensitic steels. Stabilizing the Laves phase is particularly important for the Cr-Mo systems, because $\mathrm{Fe}_{2} \mathrm{Mo}$ are easily coarsened compared to $\mathrm{Fe}_{2} \mathrm{~W}$ at $\sim 600{ }^{\circ} \mathrm{C}$. Lowering both hardness and the $\mathrm{Si}$ content of high-strength martensitic steels prevent excess hardening due to the Laves phase, which also mitigate the degradation.

5) Online monitoring of the creep curves and the $Q, V$, and $C$ analysis make it possible to detect signs for long-term unexpected degradation around targeted conditions within a relatively short time period. 


\section{List of Symbols and Abbreviations}

$\varepsilon \quad$ Strain

$\varepsilon_{i} \quad$ Strain of $i^{\text {th }}$ in Equation 8

$\dot{\varepsilon} \quad$ Creep rate

$\lambda \quad$ Maximum distance that a dislocation can move from a start point to the next stable position

$v_{e f f} \quad$ Effective attempt frequency per unit time to overcome the obstacles

$\rho \quad$ Dislocation density

$\rho_{o b} \quad$ Observable dislocation density in sub-grains

$\rho_{z} \quad$ Number density of Z-phase

$\sigma \quad$ Applied stress

$\sigma_{b} \quad$ Back stress

$\Delta S \quad$ Entropy change in the activation process

ASME The American Society of Mechanical Engineering

$b \quad$ Length of the Burgers vector

C Larson and Miller (L-M) constant

$C_{S} \quad$ L-M constant calculated based on strain rate

$C_{M G} \quad$ Monkman-Grant constant

DFRP Dissolving and finely re-precipitation

EL Rupture elongation

EBW Electron beam welding

GTAW Gas tungsten arc welding

HAZ Heat affected zone

HDZ Heterogeneous deformed zone

HRZ Heterogeneously recovered zone

HRHD Heterogeneous recovery and heterogeneous deformation

$M \quad$ Factor conversion from shear strain to tensile strain

MCR Minimum creep rate

MTD Minimum creep time for detectable degradation as compared with the shorter time data group.

MX Carbonitride of $(\mathrm{Cr}, \mathrm{Nb}, \mathrm{V})(\mathrm{C}, \mathrm{N})$ type

NIMS National Institutes for Materials Science in Tsukuba, Japan

NSR Strain rate at a specific strain is divided by each $M C R$

PAGB Prior austenitic grain boundary

PWHT Post weld heat treatment

$Q \quad$ Activation energy

$Q_{D} \quad$ Activation energy for diffusion

$Q_{S} \quad$ Activation energy calculated based on strain rate

[Q] $\quad Q / 2.3 R T$

$[Q]_{s} \quad Q_{s} / 2.3 R T$

QVC $Q, V$, and $C$

$[Q V C]_{s} \quad Q_{s}, V_{s}$, and $C_{s}$

$R \quad$ Gas constant

$R A \quad$ Reduction of area 


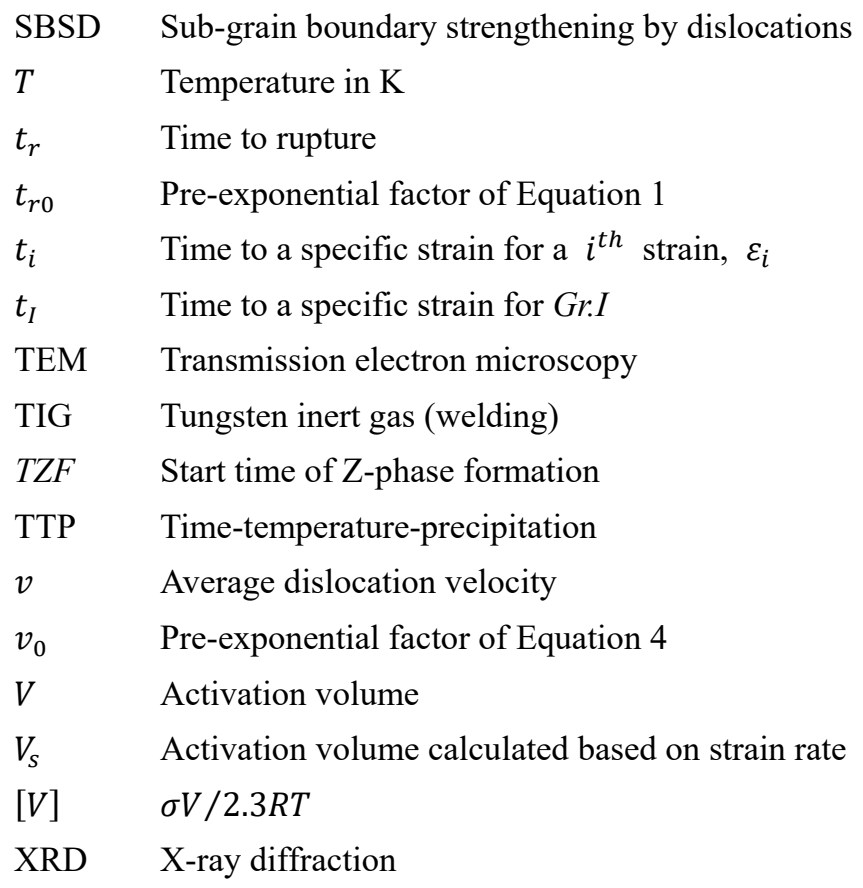

\section{Conflict Interests Statement}

The authors declare that there is no conflict of interests regarding the publication of this paper.

\section{References}

Abe, F., Araki, H., \& Noda, T. (1991). The effect of tungsten on dislocation recovery and precipitation behavior of low-activation martensitic $9 \mathrm{Cr}$ steels. Metallurgical Transactions A, 22A, 1991-2225. https://doi.org/10.1007/BF02664988

Abe, F., Nakazawa, S., Araki, H., \& Noda, T. (1992). The role of microstructure instability on creep behavior of a martensitic 9Cr-2W steel. Metallurgical Transactions A, 23, 469-477. https://doi.org/10.1007/BF02801164

Abe, F. (2003). Effect of quenching, tempering, and cold rolling on creep deformation behavior of a tempered martensitic 9Cr-1W steel. Metallurgical and Materials Transactions A, 34, 913-925. https://doi.org/10.1007/s11661-003-0222-x

Abe, F. (2005). Effect of fine precipitation and subsequent coarsening of Fe2W laves phase on the creep deformation behavior of tempered martensitic $9 \mathrm{Cr}-\mathrm{W}$ steels. Metallurgical and Materials Transactions A, 36, 321-332. https://doi.org/10.1007/s11661-005-0305-y

Abe, F. (2006a). Metallurgy for long-term stabilization of ferritic steels for thick section boiler components in USC power plant at $650{ }^{\circ} \mathrm{C}$. In Proceedings of the 8th Liege Conference on Materials for Advanced Power Engineering, 2006, September 18-20, 2006, Liege, Belgium (pp. 965-980). Forschungszentrum Juelich, Germany. Retrieved from https://www.fz-juelich.de/zb/DE/Home/home_node.html

Abe, F. (2006b). Present status of advanced high-Cr ferritic heat resistant steels for ultra-supercritical power plants. Bulletin of the Iron and Steel Institute of Japan, 11, 197-207. Retrieved from https://www.isij.or.jp/

Abe, F. (2008). Precipitate design for creep strengthening of $9 \% \mathrm{Cr}$ tempered martensitic steel for ultra-supercritical power plants. Science and Technology of Advanced Materials, 9(013002), 1-15. https://doi.org/10.1088/1468-6996/9/1/013002

Abe, F. (2009). Analysis of creep rates of tempered martensitic $9 \% \mathrm{Cr}$ steel based on microstructure evolution. Materials Science and Engineering A, 510-511, 64-69. https://doi.org/10.1016/j.msea.2008.04.118

Abe, F. (2011). Effect of Boron on microstructure and creep strength of advanced ferritic power plants steels. Procedia Engineering, 10, 94-99. https://doi.org/10.1016/j.proeng.2011.04.018

Abe, F., Ohba, T., Miyazaki, H., Toda, Y., \& Tabuchi, M. (2018). Long-term creep strength and rupture ductility of W-Mo-balanced 9Cr steel. In Proceedings of 44th MPA-Seminar, October 17-18, 2018, Stuttgart, Germany (pp. 1-15). Retrieved from https://seminar.mpa.uni-stuttgart.de/s-2018/ SitePages/Program.aspx 
Abson, D. J., \& Rothwell, J. S. (2013). Review of type IV cracking of weldments in 9-12\%Cr creep strength enhanced ferritic steels. International Materials Reviews, 58, 437-473. https://doi.org/10.1179/1743280412Y.0000000016

Arai, H. (1970). A theoretical study on the formation of carbide and chromium-depleted-layer in austenitic stainless steel. Tetsu-to-Hagane, 56, 44-54. https://doi.org/10.2355/tetsutohagane1955.56.1_44

Azuma, T., Miki, K., Tanaka, Y., \& Ishiguro, T. (2002). Effect of B on microstructural change during creep deformation in high $\mathrm{Cr}$ ferritic heat resistant steel. Tetsu-to-Hagane, 88, 678-685. https://doi.org/10.2355/tetsutohagane1955.88.10_678

Brailsford, A. D., \& Aaron, H. B. (1969). Growth of grain-boundary precipitates. Journal of Applied Physics, 40, 1702-1710. https://doi.org/10.1063/1.1657835

Cipolla, L., Danielsen, H. K., Venditti, D., Nunzio, P. E. D., Hald, J., \& Somers, M. A. J. (2010). Conversion of MX nitrides to Z-phase in a martensitic $12 \% \mathrm{Cr}$ steeel. Acta Materialia, 58, 669-679. https://doi.org/10.1016/j.actamat.2009.09.045

Cottrell, A. H. (1964). Theory of Crystal Dislocations (pp. 6-7). New York, NY: Gordon and Breach. Retrieved from https://www.amazon.com/Theory-Crystal-Dislocations-H-Cottrell/dp/B0000CMG27

Danielsen, H. K. (2007). Z-Phase in 9-12\%Cr steels (Dissertation of Ph. D.). Technical University of Denmark, Lyngby, Denmark. Retrieved from https://backend.orbit.dtu.dk/ws/files/4899462/HilmarThesis.pdf

Danielsen, H. K., \& Hald, J. (2009). Influence of Z-phase on long-term creep stability of martensitic 9 to $12 \% \mathrm{Cr}$ steels. VGB PowerTechnology, 5, 68-73. Retrieved from https://www.vgb.org/vgbmultimedia/ danielsen_pt09_05-p-3571.pdf

Di-Gianfrancesco, A., Vipraio, S. T., \& Venditti, D. (2013). Long-term microstructural evolution of 9-12\%Cr steel Grades for steam power generation plants. Procedia Engineering, 55, 27-35. https://doi.org/10.1016/j.proeng.2013.03.214

Dimmler, G., Weinert, P., Kozeschnik, E., \& Cerjak, H. (2003). Quantification of the laves phase in advanced 9-12\% $\mathrm{Cr}$ steels using a standard SEM. Materials Characterization, 51, 341-352. https://doi.org/10.1016/j.matchar.2004.02.003

Dudko, V., Belyakov, A., Molodov, D., \& Kaibyshev, R. (2013). Microstructure evolution and pinning of boundaries precipitates in a 9 pct $\mathrm{Cr}$ heat resistant steel during creep. Metallurgical and Materials Transactions A, 44A, S162-S172. https://doi.org/10.1007/s11661-011-0899-1

Dudova, N., Plotnikova, A., Molodov, A., Belyyakov, A., \& Kaibyshev, R. (2012). Structural changes of tempered martensitic $9 \% \mathrm{Cr}-2 \% \mathrm{~W}-3 \% \mathrm{Co}$ steel during creep at $650{ }^{\circ} \mathrm{C}$. Materials Science and Engineering: A, 534, 632-639. https://doi.org/10.1016/j.msea.2011.12.020

Ennis, P. J., Zielinska-Lipiec, A., Wachter, O., \& Czyrska-Filemonowicz, A. (1997). Microstructural stability and creep rupture strength of the martensitic steel P92 for advanced power plant. Acta Materialia, 45, 4901-4907. https://doi.org/10.1016/S1359-6454(97)00176-6

Ennis, P. J., Zielinska-Lipiec, A., \& Czyrska-Filemonowicz, A. (2000). Quantitative microscopy and creep strength of $9 \%$ chromium steels for advanced power stations. In A. Strang, W. M. Bank, R. D. Conroy, G. M. McColvin, J. C. Neal, and S. Simpson (Eds.), Proceedings of 5th International Charles Parsons Turbine Conference, 3-7 July, 2000, Cambridge, (pp. 498-507). London, JOM Communication Ltd. Retrieved from https://catalyst.library.jhu.edu/catalog/bib_2158972

Ennis, P. J., \& Czyrska-Filemonowicz, A. (2003). Recent advances in creep-resistant steels for power plant applications. Sadhana, 28, 709-730. https://doi.org/10.1007/BF02706455

Esherby, J. D. (1956). The continuum theory of lattice defects. In F. Seitz \& D. Turnbull (Eds.), Solid State Physics: Advances in Research and Applications (Vol. 3, pp. 79-145). NY: Academic Press Inc. https://doi.org/10.1016/S0081-1947(08)60132-0

Fedorova, I., Kipelova, A., Belyakov, A., \& Kaibyshev, R. (2013). Microstructure evolution in an advanced 9 pct $\mathrm{Cr}$ martensitic steel during creep at $923 \mathrm{~K}\left(650^{\circ} \mathrm{C}\right)$. Metallurgical and Materials Transactions A, 44A, S128-S135. https://doi.org/10.1007/s11661-012-1182-9

Fedoseeva, A., Dudova, N., \& Kaibyshev, R. (2016). Creep strength breakdown and microstructure evolution in a 3\%Co modified P92 steel. Materials Science \& Engineering A, 654, 1-12. https://doi.org/10.1016/j.msea.2015.12.027 
Fedoseeva, A., Nikitin, I., Dudova, N., \& Kaibyshev, R. (2019). Creep behavior and microstructure of a prospective Re-containing $10 \% \mathrm{Cr}-3 \% \mathrm{Co}-3 \% \mathrm{~W}$ martensitic steel. In Jiont EPRE-123HiMAT International Conference on Advances in High-Temperature Materials: Proceedings from EPRI's 9th International Conference on Advances in Materials Technology for Fossil Power Plants and the 2nd International 123HiMAT Conference on High-Temperature Materials, Octorber 21-24, 2019, Nagasaki, Japan, ASM International (pp. 217-226). Retrieved http://www.123himat-2019.mtl.titech.ac.jp/Leaflet_FinalProgram_191015_FinalVersion.pdf

Fujita, T., Yamashita, K., \& Miyake, H. (1980). The effect of nickel and cobalt on elevated temperature properties and microstructures of $10 \mathrm{Cr}-2 \mathrm{Mo}$ heat resisting steels. Transactions ISIJ, 20, 384-391. https://doi.org/10.2355/isijinternational1966.20.384

Gao, Q., Zhang, Y. Zhang, H., Li, H., Qu, F., Zhan, J., Lu, C., Wu, B., Lu, Y., \& Ma, Y. (2017). Coarsening behavior of M23C6 carbides in creep-resistant steel exposed to high temperatures. Scientific Reports, 7 , Articl No. 5859. Retrieved from https://www.nature.com/articles/s41598-017-06191-2

Garofalo, F. (1963). An empirical relationship defining the stress dependence of minimum creep rate in metals. Transactions of the metallurgical Society of AIME, 227, 351-356. Retrieved from https://catalog.princeton.edu/catalog/700074

Glen, J. (1958). A new approach to the problem of creep. Journal of the Iron and Steel Institute, 189, 333-343. Retrieved from https://iss.ndl.go.jp/books/R100000002-I000000124643-00

Gu, Y., West, G. D., \& Thomson, R. C. (2014). Investigation of creep damage and cavitation mechanism in P92 steels. In Advances in Materials Technology for Fossil Power Plants: Proceedings from the 7th International Conference (EPRI2013), October 22nd-25th, Hawaii, USA. ASM International, (pp. 596-606). Retrieved from

https://repository.lboro.ac.uk/articles/Investigation_of_creep_damage_and_cavitation_mechanisms_in_P92 _steels/9233744

Haetterstrand, M., \& Andren, H.-O. (2001). Evaluation of particle size distribution of precipitates in a 9\% chromium steel using energy filtered transmission electron microscopy. Micron, 32, 789-797. https://doi.org/10.1016/S0968-4328(00)00086-X

Hald, J. (2008). Microstructure and long-term creep properties of 9-12\% Cr steels. International Journal of Pressure Vessels and Piping, 85, 30-37. https://doi.org/10.1016/j.jpvp.2007.06.010

Hasegawa, Y., Ohgami, M., \& Muraki, T. (2003). Grain boundary strengthening mechanism of tungsten containing 9 to $12 \%$ chromium ferritic heat resistant steels at $6500^{\circ} \mathrm{C}$. Journal of the Society of Materials Science, Japan, 52, 843-850. https://doi.org/10.2472/jsms.52.843

Hasegawa, Y., Muraki, T., Yoshida, S., Ohgami, M., Okayama, Y., Kawazoe, F., \& Umeki, S. (2004). Alloy design of nano-scale precipitates bearing high strength ferritic heat resistant steels. Shinnittetsu Giho, No.381, 61-65. Retrieved from https://www.nipponsteel.com/tech/report/nsc/

Hasegawa, Y. (2014). Grade 92 creep-strength-enhanced ferritic steel. In A. Shibli (Ed.), Coal Power Plant Materials and Life Assessment - Development and Applications (pp. 52-86). Cambridge, UK, Woodhead Publishing. https://doi.org/10.1533/9780857097323.1.52

Hashizume, R., Tamura, O., Miki, K., Azuma, T., Ishiguro, T., Murata, Y., \& Morinaga, M. (2009). Benefitical effect of Re on the long-term creep strength of high $\mathrm{Cr}$ ferritic heat resistant steels. Tetsu-to-Hagane, 95, 176-185. https://doi.org/10.2355/tetsutohagane.95.176

Hassan, B., \& Corney, J. (2017). Grain boundary precipitation in Inconel 718 and ATI 718Plus. Materials Science and Technology, 33, 1879-1889. https://doi.org/10.1080/02670836.2017.1333222

Hayakawa, H., Terada, D., Yoshida, F., Nakashima, H., \& Goto, Y. (2003a). Evaluation of mobile dislocation density of modified 9Cr-1Mo steel by stress change test. Tetsu-to-Hagane, 89, 1076-1081. https://doi.org/10.2355/tetsutohagane1955.89.10_1076

Hayakawa, H., Terada, D., Yoshida, F., Nakashima, H., \& Goto, Y. (2003b). Characterization of creep deformation mechanism and evaluation of dislocation mobility of modified $9 \mathrm{Cr}-1 \mathrm{Mo}$ steel by stress change test. Journal of Japan Institute of metals, 67, 22-26. https://doi.org/10.2320/jinstmet1952.67.1_22

Hayakawa, H., Nakashima, S., Kusumoto, J., Kanaya, A., Terada, D., Yoshida, F., \& Nakashima, H. (2007a). Evaluation of creep deformation mechanism of heat resistant steel by stress change test. In Proceedings of 
CREEP8, 8th International conference on Creep and Fatigue at Elevated Temperatures, July 22-26, 2007, San Antonio, Texas (CREEP2007-26501, pp. 1-10). ASME. Retrieved from https://asmedigitalcollection.asme.org/proceedings

Hayakawa, H., Terada, D., Yoshida, F., Nakashima, H., Kanaya, A., \& Nakashima, S. (2007b). Characterization of creep deformation of $2.25 \mathrm{Cr}-1 \mathrm{Mo}$ steel by stress change test. Tetsu-to-Hagane, 93, 466-471. https://doi.org/10.2355/tetsutohagane.93.466

Hongo, H., Tabuchi, M., Li, Y., \& Takahashi, Y. (2009). Creep damage behavior of mod. 9Cr-1Mo steel welded joint. Journal of the Society of Materials Science, Japan, 58, 101-107. https://doi.org/10.2472/jsms.58.101

Hori, S., \& Saji, S. (1981). Grain boundary reaction. Bulletine of Japan Institute of Metals, 20, 863-869. https://doi.org/10.2320/materia1962.20.863

Hosoi, Y., Wade, N., Kunimitsu, S., \& Urita, T. (1986). Precipitation behavior of Laves phase and its effect on toughness of $9 \mathrm{Cr}-2 \mathrm{Mo}$ ferritic-martensitic steel. Journal of Nuclear materials, 141-143, 461-467. https://doi.org/10.1016/S0022-3115(86)80083-6

Hu, P., Yan, W., Sha, W., Wang, W., Guo, Z., Shan, Y, \& Yang, K. (2009). Study on Laves phase in an advanced heat-resistant steel. Frontier Materials Science, China, 3, 434-441. https://doi.org/10.1007/s11706-009-0063-7

Jiang, J., Zhu, L., \& Wang, Y. (2013). Hardness variation in P92 heat-resistant steel based on microstructural evolution during creep. Steel Research International, 84, 732-739. https://doi.org/10.1002/srin.201200265

Kabadwal, A., Tamura, M., Shinozuka, K., \& Esaka, H. (2010). Recovery and precipitate analysis of 9 pct Cr-1 pct $\mathrm{MoVNb}$ steel during creep. Metallurgical Transactions A, 41A, 364-379. https://doi.org/10.1007/s11661-009-0094-9

Karashima, S., Iikubo, T., Watanabe, T., \& Oikawa, H. (1971). Transmission electron microscopy of substructures developed during high-temperature creep in alpha-iron. Transactions of JIM, 12, 369-374. https://doi.org/10.2320/matertrans1960.12.369

Karashima, S., Iikubo, T., \& Oikawa, H. (1972). On the high-temperature creep behavior and substructures in alpha-iron single crystal. Transactions of JIM, 13, 176-181. https://doi.org/10.2320/matertrans1960.13.176

Kimura, K., Suzuki, K., Toda, Y., Kushima, H., \& Abe, F. (2002). Precipitation of Z-phase and degradation behavior of mod.9Cr-1Mo steel. In Proceedings of the 7th Liege Conference on Materials for Advanced Power Engineering 2002, (pp. 1171-1180). Forschungszentrum Juelich, Germany. Retrieved from https://www.fz-juelich.de/zb/DE/Home/home_node.html

Kimura, K. (2009). Creep rupture life prediction of creep resistant steels. Journal of Japan Institute of metals, 73, 323-333. https://doi.org/10.2320/jinstmet.73.323

Kimura, H., Sato, T., Bergins, C., Imano, S., \& Saito, E. (2011). Development of technologies for improving efficiency of large coal-fired thermal power plants. Hitachi Review, 60(7), 365-371. Retrieved from http://www.hitachi.com/rev/pdf/2011/r2011_07_102.pdf

Kimura, K., Sawada, K., Kushima, H., \& Toda, Y. (2012). Microstructural stability and long-term creep strength of grade 91 steel. Energy Materials: Material Science and Engineering for Energy Systems, 4, 176-183. https://doi.org/10.1179/174892312X13269692038897

Kimura, K., Sawada, K., Kushima, H., \& Toda, Y. (2013). Influence of chemical composition and heat treatment on long-term creep strength of Grade 91 steel. Procedia Engineering, 55, 2-9. https://doi.org/10.1016/j.proeng.2013.03.211

Kipelova, A., Belyakov, A., \& Kaibyshev, R. (2012). Laves phase evolution in a modified P911 heat resistant steel during creep at $923 \mathrm{~K}$. Materials Science and Engineering A, 532, 71-77. https://doi.org/10.1016/j.msea.2011.10.064

Kocer, C., Abe, T., \& Soon, A. (2009). The Z-phase in 9-12\% Cr ferritic steels: A phase stability analysis. Materials Science and Engineering A, 505, 1-5. https://doi.org/10.1016/j.msea.2008.10.028

Komazaki, S. (2009). Degradation/damage measurement and life assessment of high-temperature components. Journal of the Society of Materials Science, Japan, 58, 793-800. https://doi.org/10.2472/jsms.58.793

Kubon, Z., Foldyna, V., \& Vodarek, V. (1998). Analysis of strengthening mechanisms in 9 to 12 chromium steels. In A. Strang, T. Canley, \& G. W. Greenwood (Eds.), Microstructural Stability of Creep Resistant Alloys for 
High Temperature Plant Applications (pp. 257-270). London, Institute of Materials. Retrieved from http://metal2012.tanger.cz/files/proceedings/metal_00/papers/318.pdf

Kubon, Z., Foldyna, V., Hajduk, D., \& Simecek, P. (2000). Creep and Relaxation Properties of 9-12\% Cr steels. In A. Strang, W. M. Bank, R. D. Conroy, G. M. McColvin, J. C. Neal, \& S. Simpson (Eds.), Proceedings of 5th International Charles Parsons Turbine Conference, 3-7 July, 2000, Cambridge (pp. 485-497). London, JOM Communication Ltd. Retrieved from https://catalyst.library.jhu.edu/catalog/bib_2158972

Kunieda, T., Murata, Y., Morinaga, M., \& Koyama, T. (2004). Effect of refractory elements (X: Mo, W, Re) on the microstructure evaluation of $\mathrm{Fe}-8 \mathrm{Cr}-0.1 \mathrm{C}-\mathrm{X}$ martensitic steels during tempering. Materials Transactions, 45, 392-395. https://doi.org/10.2320/matertrans.45.392

Kunieda, T., Yamashita, K., Murata, Yoshinori, Koyama, T., \& Morinaga, M. (2006). Effect of rhenium addition on tungsten diffusivity in iron-chromium alloys. Materials Transactions, 47, 2106-2108. https://doi.org/10.2320/matertrans.47.2106

Kushima, H, Kimura, K., \& Abe, F. (1999). Degradation of mod. 9Cr-1Mo steel during long-term creep deformation. Tetsu-to-Hagane, 85, 841-847. https://doi.org/10.2355/tetsutohagane1955.85.11_841

Larson, F. R., \& Miller, J. (1952). A time-temperature relationship for rupture and creep stresses. Transactions of the American Institute of Mining and Metallurgical Engineers, 74, 765-775. Retrieved from https://search.lib.virginia.edu/catalog/u744571

Lee, J. S., Armaki, H. G., Maruyama, K., Muraki, T., \& Asahi, H. (2006). Causes of breakdown of creep strength in $9 \mathrm{Cr}-1,8 \mathrm{~W}-0.5 \mathrm{Mo}-\mathrm{VNb}$ steel. Materials Science and Engineering A, 428, 270-275. https://doi.org/10.1016/j.msea.2006.05.010

Lifshitz, I. M., \& Slyozov, V. V. (1961). The kinetics of precipitation from supersaturated solid solution. Journal of Physics and Chemistry of Solids, 19, 35-50. https://doi.org/10.1016/0022-3697(61)90054-3

Lim, R., Sauzay, M., Dalle, F., Tournie, I., Bonnaillie, P., \& Gourgues-Lorenzon, A.-F. (2011). Modelling and experimental study of the tertiary creep stage of Grade 91 steel. International Journal of Fracture, 169, 213-228. https://doi.org/10.1007/s10704-011-9585-y

Liu, F., Fors, D. H. R., Golpayegani, A., Andren, H.-O., \& Wahnstrom, G. (2012). Effect of boron on carbide coarsening at $873\left(600^{\circ} \mathrm{C}\right)$ in 9 to 12 pct chromium steels. Metallurgical and Materials Transactions A, 43A, 4053-4062. https://doi.org/10.1007/s11661-012-1205-6

Lundin, L., Norell, M., Andren, H.-O., \& Nyborg, L. (1997). Remanent life assessment of creep-resistant modified $12 \%$ chromium steels: microstrucural analysis and microstructural development models. Scandinavian Journal of Metallurgy, 26, 27-40. Retrieved from https://www.scimagojr.com/ journalsearch.php? $\mathrm{q}=28362 \&$ tip $=$ sid\&clean $=0$

Maddi, L., Deshmukh, G. S., Ballal, A. R., Peshwe, D. R., Paretkar, R. K., Laha, K., \& Mathew, M. D. (2016). Effect of Laves phase on the creep rupture properties of P92 steel. Materials Science \& Engineering A, 668, 215-223. https://doi.org/10.1016/j.msea.2016.05.074

Maruyama, K. (2019). Reliable evaluation of long-term creep properties at elevated temperature. Tetsu-to-Hagane, 105, 767-777. https://doi.org/10.2355/tetsutohagane.TETSU-2019-006

Maruyama, K., Sawada, K., \& Koike, J. (2001). Strengthening mechanisms of creep resistant tempered martensitic steel. ISIJ International, 41, 641-625. https://doi.org/10.2355/isijinternational.41.641

Masuyama, F. (2001). History of power plants and progress in heat resistant steels. ISIJ International, 41, 612-625. https://doi.org/10.2355/isijinternational.41.612

Medina, S. F. (1997). Determination of precipitation-time-temperature (TTP) diagrams for $\mathrm{Nb}$, Ti or $\mathrm{V}$

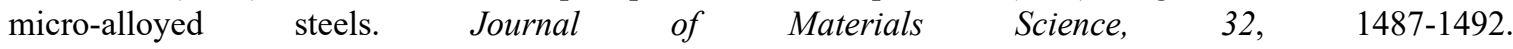
https://doi.org/10.1023/A:1018562202876

Milkereit, B., Giersberg, L., Kessler, O., \& Sciek, C. (2014). Isothermal time-temperature-precipitation diagram for an aluminium alloy 6005A by in situ DSC experiments. Materials, 7, 2631-2649. https://doi.org/10.3390/ma7042631

Monkman, F. C., \& Grant, N. J. (1956). An empirical relationship between rupture life and minimum creep rate in creep-rupture tests. Proceedings of American Society for Testing and Materials, 56, 593-620. Retrieved from http://www.astm.org/DIGITAL_LIBRARY/STP/MMR/PAGES/PRO1956-56.htm 
Morimoto, H., Ohkita, S., \& Sakurai, H. (1997). Creep rupture properties of welded joints of W containing 9Cr ferritic heat resistant steel. Quarterly Journal of the Japan Welding Society, 15, 664-673. https://doi.org/10.2207/qijwss.15.664

Morooka, S., Tomoda, Y., Adachi, Y., Morito, S., \& Kamiyama, T. (2008). Hierarchical characterization by EBSD and neutron diffraction on heterogeneous deformation behavior of a martensitic steel. Tetsu-to-Hagane, 94, 313-320. https://doi.org/10.2355/tetsutohagane.94.313

Mura, T., \& Mori, T. (1976). Micromechanics-Dislocation and Inclusions. Tokyo: Baihukan. ASIN: B000JA16HW. [In Japanese] Retrieved from https://iss.ndl.go.jp/books/R100000002-I000001129159-00

Muroki, M. (2017). The way to high efficiency boilers for power plant led by Ni-based alloy: from $600{ }^{\circ} \mathrm{C}$-class ultra-super critical (USC) boilers to $700{ }^{\circ} \mathrm{C}$-class advanced ultra-super-critical (A-USC) boilers. IHI Engineering Review, 50(1), 26-29. Retrieved from http://www.ihi.co.jp/en/

Nie, M., Zhang, J., Huang, F., Liu, J. W., Zhu, X. K., Chen, Z. L., \& Ouyang, L. Z. (2014). Microstructure evolution and life assessment of P92 steel during long-term creep. Journal of Alloys and Compounds, 588, 348-356. https://doi.org/10.1016/j.jallcom.2013.11.080

NIMS: Creep Data Sheet on Creep Deformation Properties of 9Cr-1Mo-V-Nb Steel Tubes for Boilers and Heat Exchangers and 9Crt-1Mo-V-Nb Steel Plate for Boilers and Pressure Vessels, No. D-1. (2007). Tsukuba, Japan. Retrieved from http://www.nims.go.jp/mits/english/creep_lst._e.htm

NIMS: Data Sheets on the Elevated-Temperature Properties of $11 \mathrm{Cr}-2 \mathrm{~W}-0.4 \mathrm{Mo}-1 \mathrm{Cu}-\mathrm{Nb}-\mathrm{V}$ stainless steel pipe for high temperature service (KA-SUS $410 \mathrm{~J} 3 \mathrm{TP}$ ), $11 \mathrm{Cr}-2 \mathrm{~W}-0.4 \mathrm{Mo}-1 \mathrm{Cu}-\mathrm{Nb}-\mathrm{V}$ stainless steel plate for power plants (KA-SUS 410J3), and $11 \mathrm{Cr}-2 \mathrm{~W}-0.4 \mathrm{Mo}-1 \mathrm{Cu}-\mathrm{Nb}-\mathrm{V}$ stainless steel tube for power boilers (KA-SUS $410 \mathrm{~J} 3 \quad \mathrm{~TB}$ ), No. 51A. (2013). Tsukuba, Japan. Retrieved from http://www.nims.go.jp/mits/english/creep_lst._e.htm

NIMS: Data Sheets on the Elevated-Temperature Properties of 9Cr-1Mo-V-Nb Steel Tubes for Boilers and Heat Exchangers, 9Crt-1Mo-V-Nb Steel Plates for Boilers and Pressure Vessels, and 9Crt-1Mo-V-Nb Steel Seamless Pipe for High Temperature Service, No. 43A. (2014). Tsukuba, Japan. Retrieved from http://www.nims.go.jp/mits/english/creep_lst._e.htm

NIMS: Data Sheets on the Elevated-Temperature Properties of 9Cr-0.5Mo-1.8W-V-Nb Steel Tubes for Power Boilers and 9Cr-0.5Mo-1.8W-V-Nb Steel Pipe for High Temperature Service, No. 48B. (2018). Tsukuba, Japan. Retrieved from http://www.nims.go.jp/mits/english/creep_lst._e.htm

Norton, F. H. (1929). The Creep of Steel at High Temperatures. NY: McGraw-Hill Book Co. Retrieved from https://archive.org/details/creepofsteelathi00nort

Ogata, T., Sakaki, T., \& Yaguchi, M. (2009). Uniaxial creep rupture property of mod. 9Cr-1Mo steel welded joint and proposal of creep damage evaluation method. Journal of the Society of Materials Science, Japan, 58, 94-100. https://doi.org/10.2472/jsms.58.94

Oikawa, H. (1982). Lattice self-diffusion in solid iron: A critical review. Technology Reports of the Tohoku University, 47, 67-77. Retrieved from http://www.worldcat.org/title/technology-reports-of-thetohoku-university/oclc/2451160

Orowan, E. (1940). Problems of plastic gliding. Proceedings of the Physical Society, 52, 8-22. https://doi.org/10.1088/0959-5309/52/1/303

Panait, C. G., Bendick, W., Fuchsmann, A., Gourgues-Lorezon, A.-F., \& Besson, J. (2010a). Study of the microstructure of the Grade 91 steel after more than $100000 \mathrm{~h}$ of creep exposure at $600^{\circ} \mathrm{C}$. International Journal of Pressure Vessels and Piping, 87, 326-335. https://doi.org/10.1016/j.ijpvp.2010.03.017

Panait, C. G., Zielinska-Lipiec, A., Koziel, T., Czyrska-Filemonowicz, A., Gourgues-Lorenzon, A.-F., \& Bendick, W. (2010b). Evolution of dislocation density, size of subgrains and MX-type precipitates in a P91 steel during creep and during thermal ageing at $600{ }^{\circ} \mathrm{C}$ for more than $100000 \mathrm{~h}$. Materials Science and Engineering $A$, 527, 4062-4069. https://doi.org/10.1016/j.msea.2010.03.010

Parker, J. D., \& Siefert, J. A. (2018). Creep fracture in tempered martensitic steels. International Journal of Petrochemical Science \& Engineering, 3(1), 00068. https://doi.org/10.15406/ipcse.2018.03.00068

Sanhueza, J. P., Rojas, D., Garcia, J., Melendrez. M. F., Toledo, E., Montalba, C., Alvarado, M. I., \& Jaramillo, A. F. (2019). Computational modeling of the effect of $\mathrm{B}$ and $\mathrm{W}$ in the phase transformation of M23C6 carbides 
in 9 to 12 pct $\mathrm{Cr}$ martensitic/ferritic steels. Materials Research Express, 6, 1-15. https://doi.org/10.1088/2053-1591/ab500c

Sawada, K., Takeda, M., Maruyama, K., Ishii, R., Yamada, M., Nagae, Y., \& Komine, R. (1999). Effect of W on recovery of lath structure during creep of high chromium martensitic steels. Materials Science and Engineering A, 267, 19-25. https://doi.org/10.1016/S0921-5093(99)00066-0

Sawada, K., Kubo, K., \& Abe, F. (2003). Contribution of coarsening of MX carbonitrides to creep strength degradation in high chromium ferritic steel. Materials Science and Technology, 19, 732-738. https://doi.org/10.1179/026708303225010687

Sawada, K., Kushima, H., \& Kimura, K. (2006). Z-phase formation during creep and aging in 9-12\% Cr heat resistant steels. ISIJ International, 46, 769-775. https://doi.org/10.2355/isijinternational.46.769

Sawada, K., Kushima, H., Kimura, K., \& Tabuchi, M. (2007). TTP diagram of Z phase in 9-12\% Cr heat-resistant steels. ISIJ International, 47, 733-739. https://doi.org/10.2355/isijinternational.47.733

Sawada, K., Suzuki, K., Kushima, H., Tabuchi, M., \& Kimura, K. (2008). Effect of tempering temperature on Z-phase formation and creep strength in $9 \mathrm{Cr}-1 \mathrm{Mo}-\mathrm{V}-\mathrm{Nb}-\mathrm{N}$ steel. Material Science and Engineering A, 480, 558-563. https://doi.org/10.1016/j.msea.2007.09.031

Sawada, K., Bauer, M., Kauffmann, F., Mayr, P., \& Klenk, A. (2010). Microstructural change of 9\% Cr-welded joints after long-term creep. Material Science and Engineering A, 527, 1417-1426. https://doi.org/10.1016/j.msea.2009.10.044

Sawada, K., Kushima, H., Tabuchi, M., \& Kimura, K. (2011). Microstructural degradation of Gr.91 steel during creep under low stress. Materials Science and Engineering A, 528, 5511-5518. https://doi.org/10.1016/j.msea.2011.03.073

Sawada, K., Kushima, H., Hara, T., Tabuchi, M., \& Kimura, K. (2014a). Heat-to-heat variation of creep strength and long-term stability of microstructure in Grade 91 steels. Materials Science and Engineering A, 597, 164-170. https://doi.org/10.1016/j.msea.2013.12.088

Sawada, K., Kushima, M., Tabuchi, M., \& Kimura, K. (2014b). Effect of creep deformation on Z phase formation in Gr.91 steel. Materials Science and Technology, 30, 12-16. https://doi.org/10.1179/1743284713Y.0000000309

Sawada, K., Sekido, K., Kimura, K., Arisue, K., Honda, M., Komai, N., .. \& Kubushiro, K. (2019a). Effect of initial microstructure on creep strength of ASME Grade T91 steel. Tetsu-to-Hagane, 105, 433-442. https://doi.org/10.2355/tetsutohagane.TETSU-2018-066

Sawada, K., \& Kimura, K. (2019b). Stability of long-term creep strength and microstructure in high Cr steels. Bulletin of The Iron and Steel Institute of Japan, 24, 68-72.

Schoeck, G. (1980). Thermodynamics and thermal activation of dislocations. In F. R. N. Nabarro (Ed.), Dislocations in Solids volume 3 Moving Dislocations (pp. 63-159). Amsterdam: North-Holland Pub. Co.

Sherby, O. D., Orr, R. L., \& Dorn, J. E. (1953). Creep Correlations of Metals at Elevated Temperatures in 25th Techn. Rpt. Sr. 22 Issue 25 N7-on-295 (pp. 1-44). Berkeley, CA.: University of California. Retrieved from http://www.dtic.mil/dtic/tr/fulltext/u2/005978.pdf

Shewmon, P. G. (1963). Diffusion in Solid (pp. 164-187). McGraw-Hill Book Co., New York.

Sikka, V. K., Cowgill, M. G., \& Roberts, B. W. (1983). Creep properties of modified 9 Cr-1Mo steel. In J. W. Davis \& D. J. Michel (Eds.), Proceedings of Topical Conference on Ferritic Alloys for Use in Nuclear Energy Technologies, Snowbird, Utah, June (pp. 413-423). American Nuclear Society, La Grange Park, IL. https://doi.org/10.2172/5687534

Suzuki, K., Kumai, S., Kushima, H., Kimura, K., \& Abe, F. (2000). Heterogeneous recovery and precipitation of Z-phase during long-term creep deformation of modified 9Cr-1Mo steel. Tetsu-to-Hagane, 86, 550-557. https://doi.org/10.2355/tetsutohagane1955.86.8_550

Suzuki, K., Kumai, S., Kushima, H., Kimura, K., \& Abe, F. (2003). Precipitation of Z-phase and precipitation sequence during creep deformation of mod.9Cr-1Mo steel. Tetsu-to-Hagane, 89, 691-698. https://doi.org/10.2355/tetsutohagane1955.89.6_691 
Tabuchi, M., Watanabe, T., Kubo, K., Matsui, M, Kinugawa, J., \& Abe, F. (2001). Creep crack growth behavior in the HAZ of weldments of W containing high $\mathrm{Cr}$ steel. International Journal of Pressure Vessels and Piping, 78, 779-784. https://doi.org/10.1016/S0308-0161(01)00090-4

Tamura, M., Hayakawa, H., Yoshitake, A., Hishinuma, A., \& Kondo, T. (1988). Phase stability of reduced activation ferritic steel $8 \% \mathrm{Cr}-2 \% \mathrm{~W}-0.2 \% \mathrm{~V}-0.04 \%$ Ta-Fe. Journal of Nuclear Materials, 155-157, 620-625. https://doi.org/10.1016/0022-3115(88)90384-4

Tamura, M., Esaka, H., \& Shinozuka, K. (1999). Stress and temperature dependence of time to rupture of heat resisting steels. ISIJ International, 39, 380-387. https://doi.org/10.2355/isijinternational.39.380

Tamura, M., Esaka, H., \& Shinozuka, K. (2000). Physical meaning of the new creep rupture equation for heat resisting steels. Materials Transaction, JIM, 41, 272-278. https://doi.org/10.2320/matertrans1989.41.272

Tamura, M., Esaka, H., \& Shinozuka K. (2003). Applicability of an exponential law in creep of metals. Materials Transactions, 44, 118-126. https://doi.org/10.2320/matertrans.44.118

Tamura, M., Iida, T, Kusuyama, H., Shinozuka, K., \& Esaka, H. (2004). Re-dissolution of VN during tempering in high chromium heat resistant martensitic steel. ISIJ International, 44, 153-161. https://doi.org/10.2355/isijinternational.44.153

Tamura, M., Nakamura, M., Shinozuka, K., \& Esaka, H. (2008). Tempering and Precipitation Behavior of 7 pct $\mathrm{Cr}-0.1$ pet V-0.06 pet Nb-0.08 pet N steel. Metallurgical and Materials Transactions A, 39A, 1060-1076. https://doi.org/10.1007/s11661-008-9486-5

Tamura, M., Sakasegawa, H., Shiba, K., Tanigawa, H., Shinozuka, K., \& Esaka, H. (2011). Decomposition of Y2Ti2O7 particles in 8 pct $\mathrm{Cr}$ oxide-dispersion-strengthened martensitic steel during tempering. Metallurgical and Materials Transactions A, 42, 2176-2188. https://doi.org/10.1007/s11661-011-0640-0

Tamura, M., Abe, F., Shiba, K., Sakasegawa, H., \& Tanigawa, H. (2013). Larson-Miller constant of heat-resistant steel. Metallurgical and Materials Transactions A, 44, 264-52661. https://doi.org/10.1007/s11661-013-1631-0

Tamura, M. (2015a). Method of estimating the long-term rupture strength of $11 \mathrm{Cr}-2 \mathrm{~W}-0.4 \mathrm{Mo}-1 \mathrm{Cu}-\mathrm{Nb}-\mathrm{V}$ steel. Metallurgical and Materials Transactions A, 46, 1958-1972. https://doi.org/10.1007/s11661-015-2784-9

Tamura, M., \& Abe, F. (2015b). Changes in estimated dislocation density during creep in martensitic heat-resistant steel. Journal of Materials Science Research, 4(4), 48-69. https://doi.org/10.5539/jmsr.v4n4p48

Tamura, M. (2017). Verification of equation for evaluating dislocation density during steady-state creep of metals. Journal of Materials Science Research, 6(2), 20-62. https://doi.org/10.5539/jmsr.v6n2p20

Tamura, M. (2018). Relationship between sub-grain size and dislocation density during steady-state dislocation creep of polycrystalline cubic metals. Journal of Materials Science Research, 7(4), 26-45. https://doi.org/10.5539/jmsr.v7n4p26

Tamura, M. \& Abe, F. (Fujio Abe, one of the authors is lacked in JMSR by a mistake) (2021). Analysis on degradation in creep strength of 9Cr-W martensitic steel, Journal of Materials Science Research, 10(1), 1-27. https://doi.org/10.5539/jmsr.v10n1p1

Turnbull, D., \& Fisher, J. C. (1949). Rate of nucleation in condensed system. The Journal of Chemical Physics, 17 , 71-73. https://doi.org/10.1063/1.1747055

Wagner, C. (1961). Theorie der altering von niderschlagen durch umloesen (Ostwald Reifung). Zeitschrift fur Electrochemie, 65, 581-591. Retrieved from https://www.researchgate.net/publication/230384240 Theorie_Der_Alterung_Von_Niederschlagen_Durch_Umlosen_Ostwald-Reifung

Xu, Y., Wang, M., Wang, Y., Gu, T., Chen, L., Zhou, X., Ma, Q., Liu, Y., \& Huang, J. (2015). Study on the nucleation and growth of Laves phase in a $10 \% \mathrm{Cr}$ martensite ferritic steel after long-term aging. Journal of Alloys and Compounds, 621, 93-98. https://doi.org/10.1016/j.jallcom.2014.09.204

Yan, W., Wang, W., Shan, Y.-Y., \& Yang, K. (2013). Microstrucural stability of 9-12\%Cr ferrite/martensite heat-resistant steels. Frontier Materials Science, 7(1), 1-27. https://doi.org/10.1007/s11706-013-0189-5

Yi, Y., Xu, W., Xia, F., Gang, T., \& Chen, L. (2017). Effects of alloying elements M (M=Fe, Mo) on phase stability of Cr23C6 carbides from first-principles. In Proceedings of the 2017 2nd International Conference on Advances in Materials, Mechatronics and Civil Engineering (ICAMMCE 2017). Advances in Engineering Research, 121, 74-80. https://doi.org/10.2991/icammce-17.2017.17 
Yoshinaga, H., Horita, Z., \& Kurishita, H. (1981). Dtermination of high-temperature deformation mechanism in crystalline materials by the strain-rate change test. Acta Metallurgica, 29, 1815-1824. https://doi.org/10.1016/0001-6160(81)90107-3

Yoshizawa, M., Igarashi, M., \& Nishizawa, T. (2005). Effect of tungsten on the Ostwald ripening of M23C6 carbides in martensitic heat resistant steel. Tetsu-to-Hagane, 91, 272-277. https://doi.org/10.2355/tetsutohagane1955.91.2_272

Yoshizawa, M., Igarashi, M., Moriguchi, K., Iseda, A., Armaki, H. G., \& Maruyama, K. (2009). Effect of precipitates on long-term creep deformation properties of P92 and P122 type advanced ferritic steels for USC power plants. Materials Science and Engineering A, 510-511, 162-168. https://doi.org/10.1016/j.msea.2008.05.055

Zeng, Y.-P., Jia, J.-D., Cai, W.-H., Dong, S.-Q., \& Wang, Z.-C. (2018). Effect of long-term service on the precipitates in P92 steel. International Journal of Minerals, Metallurgy and Materials, 25, 913-921. https://doi.org/10.1007/s12613-018-1640-5

Zielinski, A., Golanski, G., \& Sroka, M. (2016). Assessment of microstructure stability and mechanical properties of X10CrWMoVNb9-2 (P92) steel after long-term thermal aging for high-temperature applications. Kovove Materials, 54, 61-70. https://doi.org/10.4149/km_2016_1_61 


\section{Appendix A}

Figures Relating on Creep Curve:

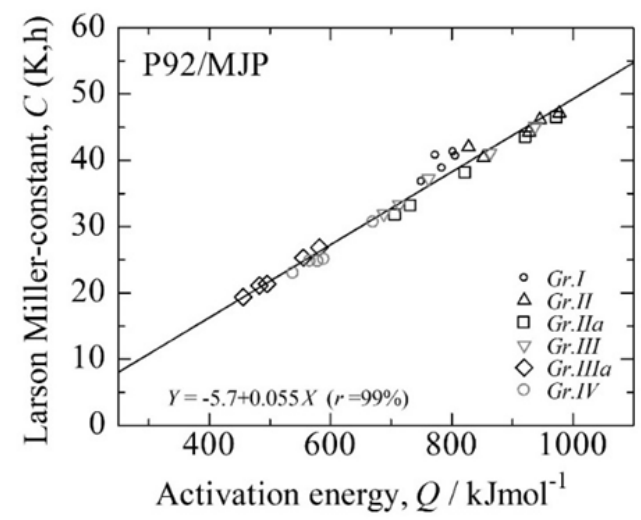

Figure A1. Relationship between $Q$ and $C$ for time to rupture of Grade 92. Definition of Gr.I etc. are explained in Section 4.3
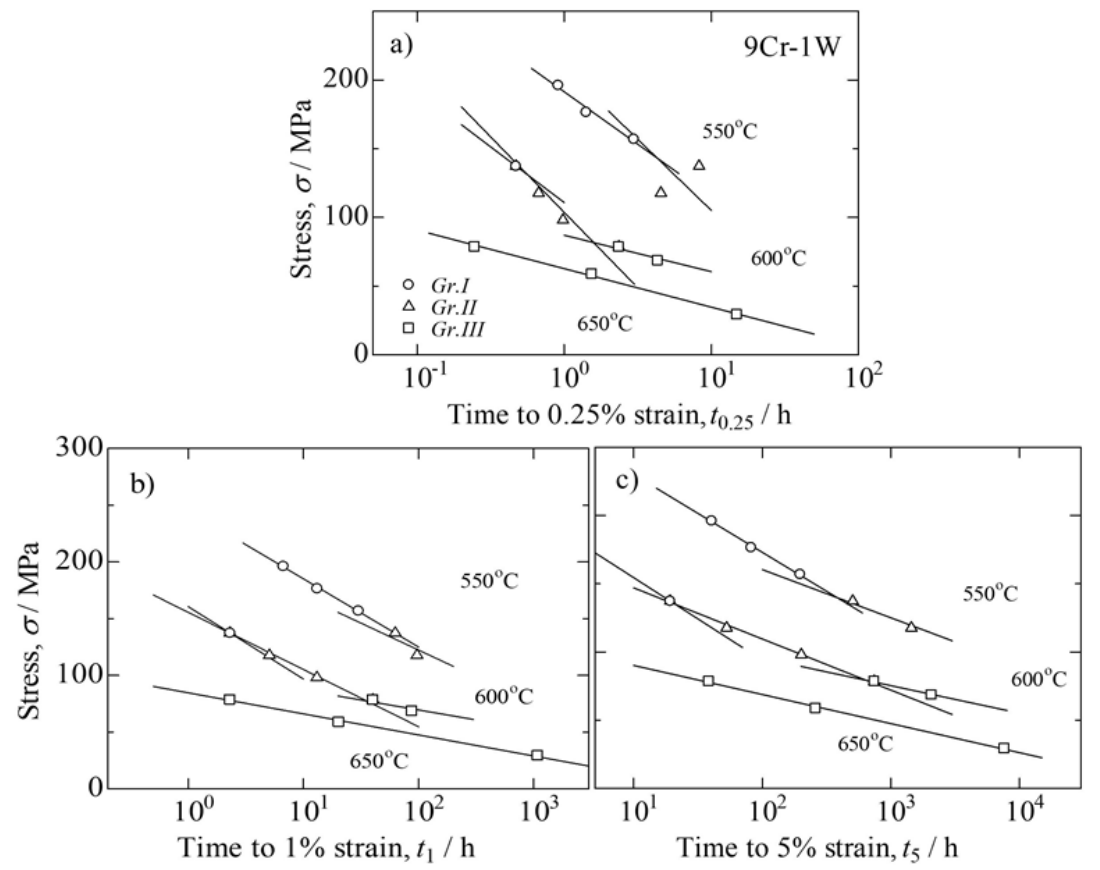

Figure A2. Stress vs. time to specific strains relationships for $9 \mathrm{Cr}-1 \mathrm{~W}$ steel; a) $0.25 \%$ strain, Z-shape, b) $1 \%$ strain, inverse J-shape, and c) 5\% strain, inverse J-shape. Definitions of each data group are the same as Figure 2 

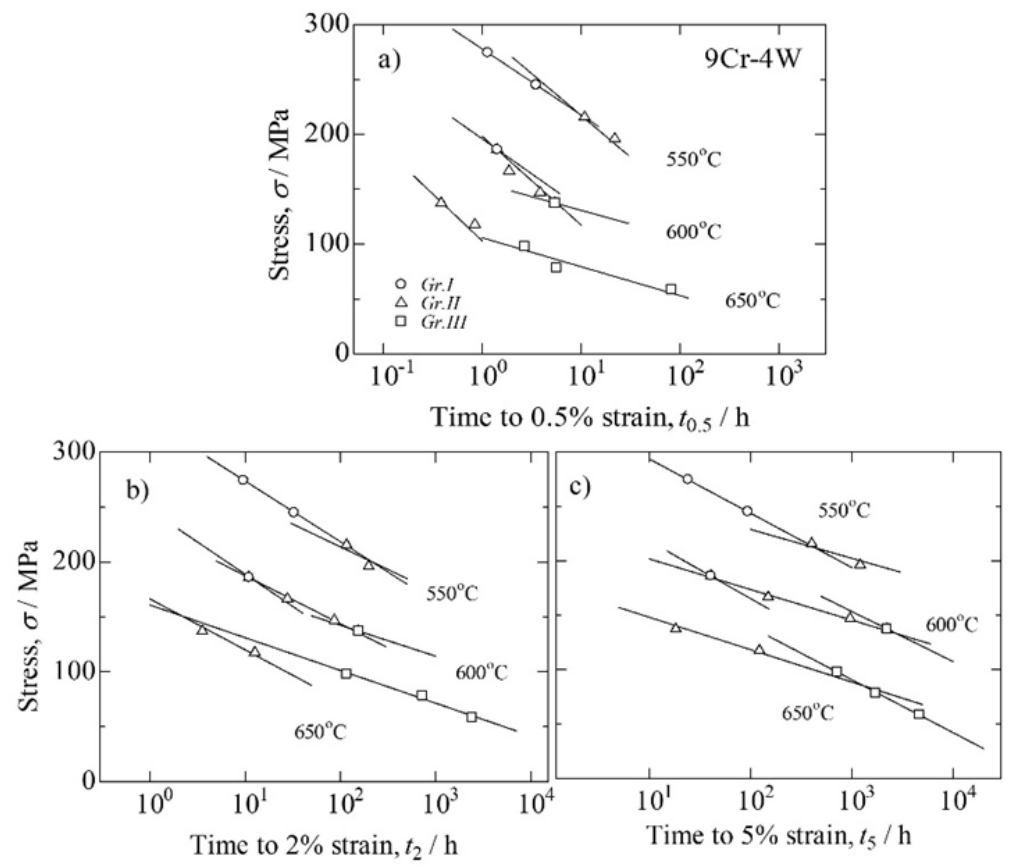

Figure A3. Stress vs. time to specific strains for $9 \mathrm{Cr}-4 \mathrm{~W}$ steel analyzed employing the exponential method shown in Section 2 and regression lines for each data group explained in Section 4.2. a) 0.5\% strain, Z-shape, b) $2 \%$ strain, inverse J-shape, and c) 5\% strain, S-shape. Definitions of each data group are the same as Figure 7

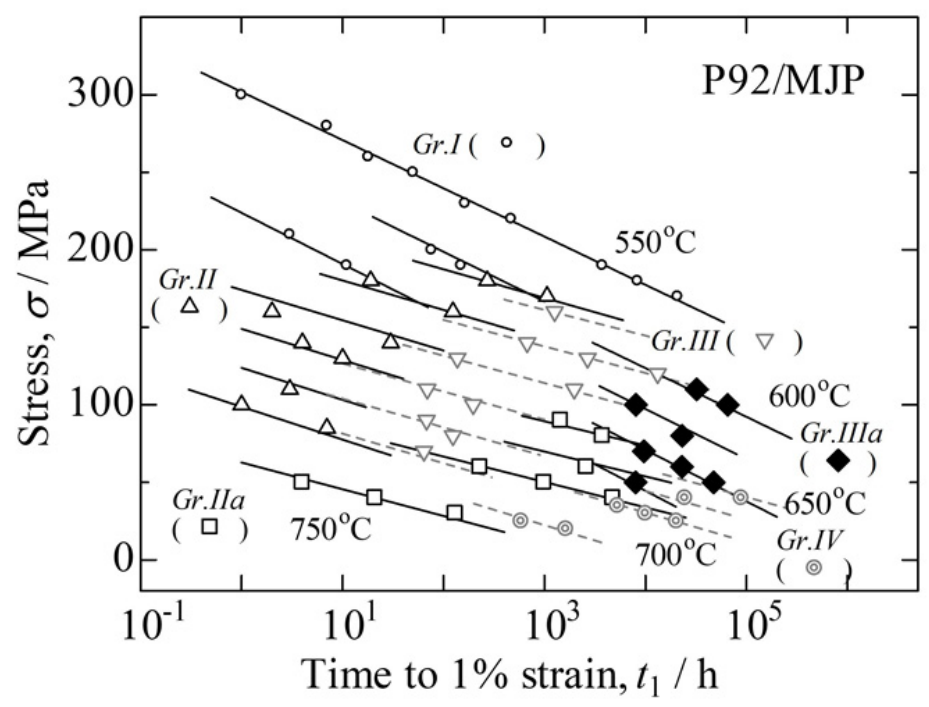

Figure A4. Stress vs. time to $1 \%$ strain relationship for P92/MJP steel. Definitions of each group are the same as in Figure 12 


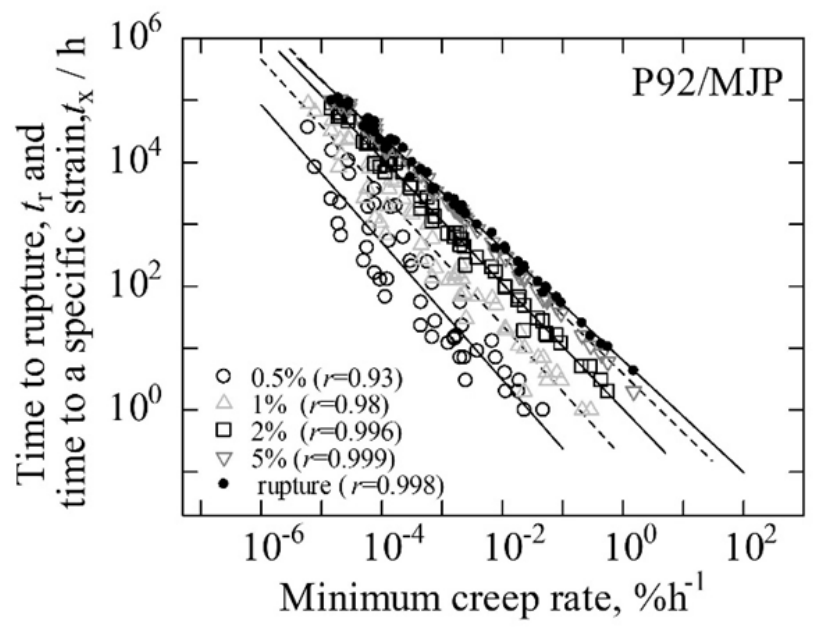

Figure A5. Monkman-Grant relationship and minimum creep rate vs. time to a specific strain relationship as indicated for P92/MJP steel and numbers in each parenthesis show each relationship coefficient in $\%, r$
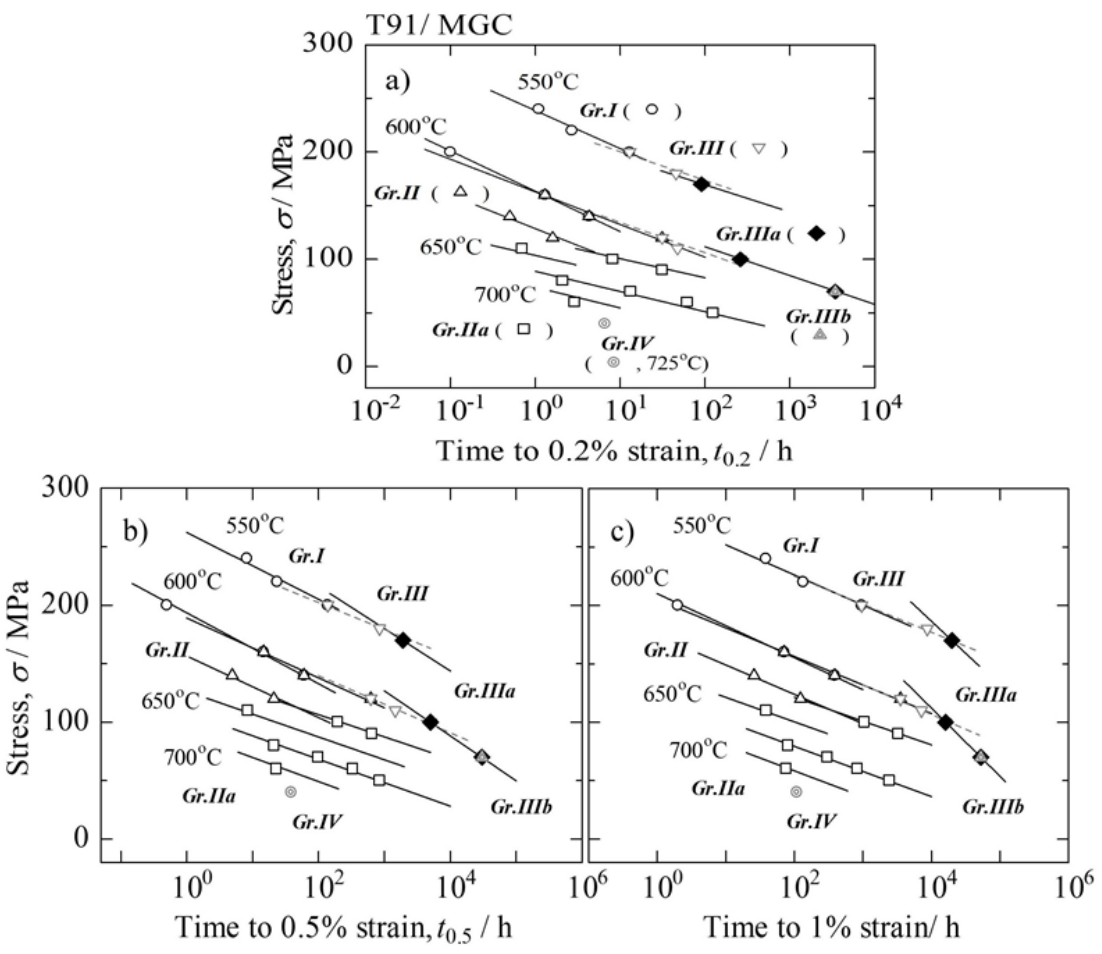

Figure A6. Stress vs. time to specific strains for T92/MJT steel analyzed employing the exponential method shown in Section 2 and regression lines for each data group explained in Section 4.4. a) 0.5\% strain, b) 1\% strain, and c) $2 \%$ strain. Isothermal lines are drawn for $t_{0.5}$ and $t_{1}$ at 550,575 , and $675{ }^{\circ} \mathrm{C}$ and $t_{0.5}$ and $t_{1}$ under 220,160 , and $80 \mathrm{MPa}$ at each temperature are shorter than those expected from $t_{0.5}$ and $t_{1}$ under higher stresses, however these abnormal phenomena are diminished after longer time creep including time to rupture

(Figure 15) 

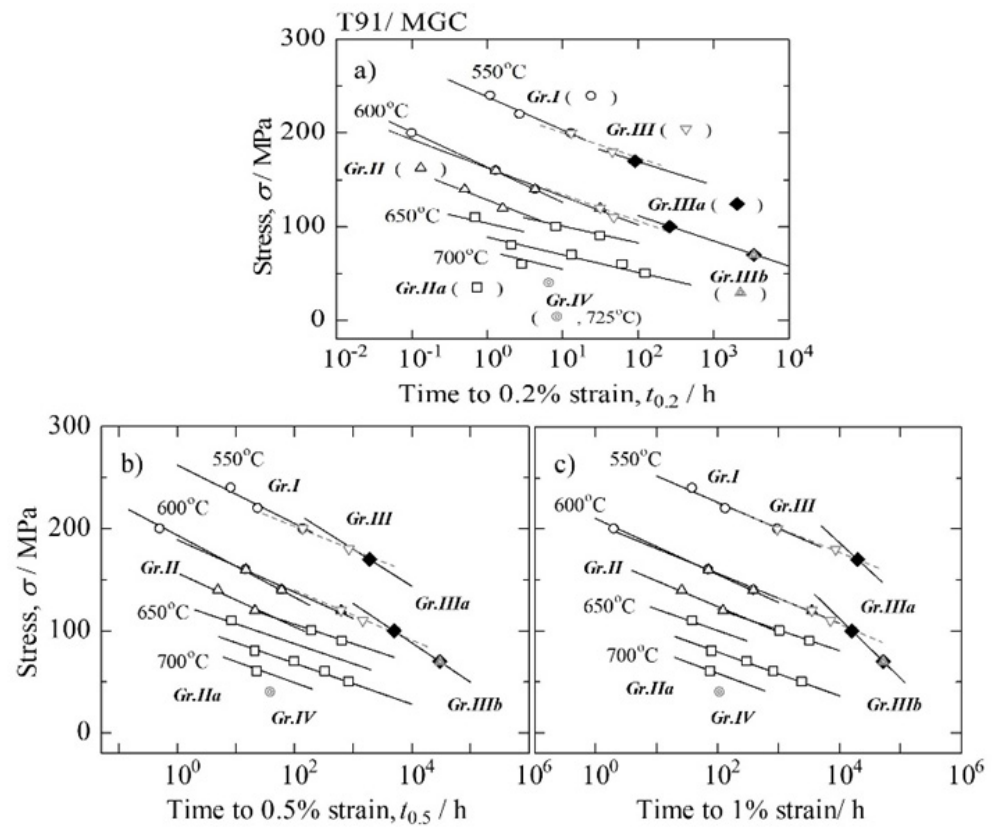

Figure A7. Stress vs. time to as specific strains for T91/MGC steel analyzed employing the exponential method shown in Section 2 and regression lines for each data group explained in Section 4.5. a) 0.2\% strain, b) 0.5\% strain, and c) $1 \%$ strain

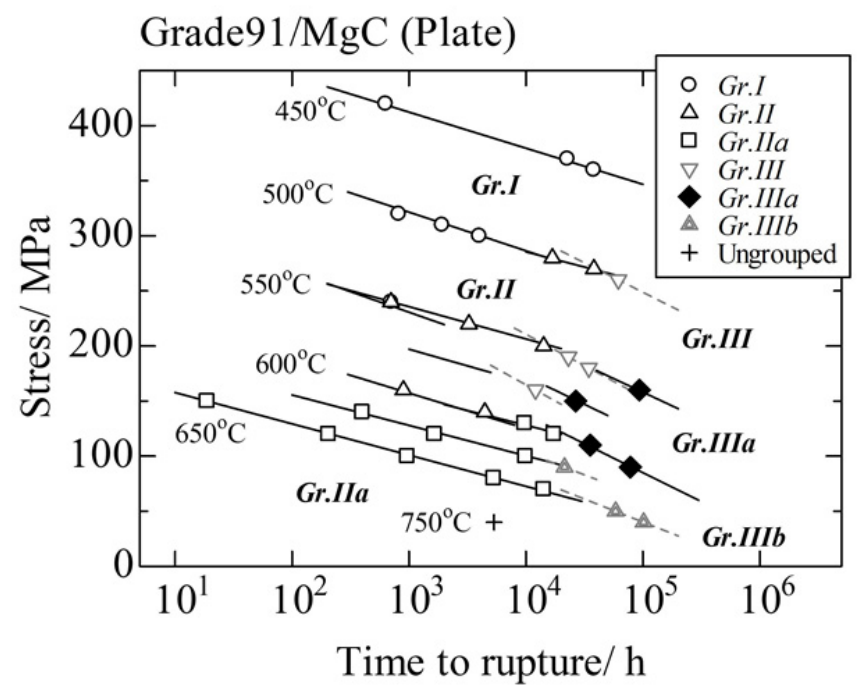

Figure A8. Stress vs. time to rupture relationship for Grade $91 / \mathrm{MgC}$. Classification for each group is determined referring those for T91/MGC 


\section{Appendix B}

Relation between Activation Energy and Back Stress:

Hayakawa et al. (2007a) performed stress dip tests on several Cr-Mo steels and showed that moving dislocations during creep drag the solute atoms of Mo. The effective stress to the dislocation is at most $10 \%$ of the applied stress, assuming that the dislocation velocity is proportional to the effective stress, where the effective stress is defined as the applied stress minus the back stress. More than $90 \%$ of the applied stress corresponds to the back stress. In our considerations, the concept of back stress is also included in the value of $Q$ as explained in Section 2.3.1. We can also estimate the back stress. It is established that creep phenomena strongly depend on self-diffusion (Sherby et al., 1953; Tamura et al., 2017). Therefore, the numerator in the exponential term of Equation 1 can be rewritten as:

$$
Q-\sigma V=Q_{D}+\sigma_{b} V-\sigma V=Q_{D}-\left(\sigma-\sigma_{b}\right) V
$$

Then, we can obtain the following relation.

$$
\sigma_{b} V=Q-Q_{D}
$$

where, $\sigma_{b}$ and $Q_{D}$ are the back stress and the activation energy for self-diffusion (Tamura et al., 2000), respectively. We can estimate the back stress at rupture for Gr.IIIa of P92/MJP as:

$$
\sigma_{b}=\left(Q-Q_{D}\right) / V=(495.4-264.7) * 1000 / 505.1 \approx 457 \mathrm{MPa}
$$

for $Q=495.4 \mathrm{kJmol}^{-1}, Q_{D} \approx 264.7 \mathrm{kJmol}^{-1}$, and $V=505.1 \mathrm{~cm}^{3} \mathrm{~mol}^{-1}$.

A similar calculation for Gr.III of P92/MJP leads to:

$$
\sigma_{b} \approx 696 \mathrm{MPa}
$$

In Section 4.3, we showed that the creep mode of MJP changes from Gr.III to Gr,IIIa at $\sim 600{ }^{\circ} \mathrm{C}$ after tens of thousands of hours. It is clear that this mode change is caused by the progressive recovery and it is reasonable that Equations A3 and A4 indicate that back stress decreases as recovery progresses.

These back stresses are much higher than the applied stress of $\sim 100 \mathrm{MPa}$. However, this is reasonable because the dislocation velocity defined by Equation 4 is positive, even if $\sigma-\sigma_{b}<0$ as long as $Q-\sigma V=Q_{D}-$ $\left(\sigma-\sigma_{b}\right) V>0$. Yoshinaga, Horita, \& Kurishita (1981) highlighted that the back stress changes every second depending on the location. Moreover, when dislocations cut each other, they temporarily experience very high stress. Therefore, there is no issue, even if the back stress exceeds the applied stress.

\section{Copyrights}

Copyright for this article is retained by the author(s), with first publication rights granted to the journal.

This is an open-access article distributed under the terms and conditions of the Creative Commons Attribution license (http://creativecommons.org/licenses/by/4.0/). 